\title{
Plain Facts about GMOs
}

\author{
Hungarian White Paper
}

Editors:

Ervin Balázs, Dénes Dudits, László Sági 
Editors:

ERVIN BALÁZS, DÉNES DUDITS, LÁSZLÓ SÁGI

Authors:

ERVIN BALÁZS, ZOLTÁN BEDÔ, ZSUZSANNA BÓSZE DÉNES DUDITS, MIKLÓS GÁBOR FÁRI, JÚLIA GIMES ELEN GÓCZA, JÁNOS GUNDEL, LÁSZLÓ HIRIPI,

LÁSZLÓ HORNOK, KORNÉL KOVÁCS, CSABA L. MARTON, LÁSZLLÓ MÁRTON, MÁRIA OSZZVALD, JÓZSEF POPP,

KATALIN POSTA, NORBERT POTORI, INGO POTRYKUS, LÁSZLÓ SÁGI, BÉLA SOMFAI, LÁSZLÓ TAMÁS, PÁL VENETIANER

Publisher's reader: ERZSÉBET FEJES

Cover design: PÉTER SÁVAI

Paper and text design: ATTILA ÁCS

Printed by: TISZA PRESS NYOMDA

\section{ISBN 978-963-08-1066-1}

Published by: Barabás Zoltán Federation of Biotechnology Dénes Dudits

This work is subject to copyright. No part of this work covered by the copyrights hereon may be reproduced or copied in any form or by any means

- translation, reprinting, reuse of illustrations, recitation,

broadcasting, reproducing on microfilms or in other ways, and storage in data bank - without written permission of the publisher

(C) Barabás Zoltán Federation of Biotechnology

(C) Dénes Dudits

\section{Table of contents}

1. Recommendation Ingo Potrykus

4

2. Editor's introduction Ervin Balázs, Dénes Dudits, László Sági .... $\odot$

3. Statement on Genetically Modified Organisms from the Section of Agricultural Sciences of the Hungarian Academy of Sciences .... $\odot \quad 8$

4. Position of gene technology amongst genetic modification tools Pál Venetianer

5. Procedures for the generation of genetically modified organisms László Sági, Elen Gócza, Kornél Kovács

6. Integration of gene technology research into plant breeding Zoltán Bedố, Csaba L. Marton.

7. Present and future role of different generations of GM crops in the Hungarian agriculture Dénes Dudits

8. The results and future prospects of genetically modified livestock animals Zsuzsanna Bósze, László Hiripi ............................ $\odot 49$

9. GM plants for feeding livestocks János Gundel ............................. $\odot 58$

10. The possible role of GM technology in the breeding, propagation and processing of the future biomass crops László Márton, Miklós G. Fári

11. Biopesticides and biofertilizers László Hornok, Katalin Posta .. $\rightleftharpoons 71$

12. The role of GM microbes in the fermentation technology Kornél Kovács ....

13. Genetically modified plants as the basis of food quality improvement and pharmaceutical production

László Tamás, Mária Oszvald

14. An overview of the economic impacts of GM crops József Popp, Norbert Potori

15. GMOs and environmental safety

Ervin Balázs, László Sági

16. The process of gene technology regulation Ervin Balázs ........ $\rightarrow 105$

17. GM plants - the media - the public Júlia Gimes ........................... $\Rightarrow \quad 110$

18. Genetically modified crops: A beginning or the end? Béla Somfai ....

19. Reference list of Hungarian research papers describing GMOs.

20. List of authors and their contact details 


\section{Recommendation}

\section{INGO POTRYKUS}

The Hungarian scientific GMO experts have compiled peer-reviewed data - 'plain facts' - about a controversial issue in European politics. In contrast to the 'opinions' based on so-called 'information' (without any exception all falsified by careful scientific analysis) offered by GMO-opponents since decades (and dominating the view of many decision makers and the media), these facts represent reliable and solid information. They are in agreement with the conclusions drawn by a group of international experts, which met in the Vatican, upon invitation by the Pontifical Academy of Sciences, for a 'study week' to analyse the present 'state-of-art' of GMO science. The published 'Statement' fully supports the Hungarian GMO white paper and at the same time totally contradicts the present wide-spread negative European attitude towards GMOs. As a member both of the Pontifical Academy of Sciences as well as of the Hungarian Academy of Sciences I would like to strongly recommend decision makers and media representatives to study the facts presented, and to reconsider whether it is wise politics to follow the recommendations of activists (who may have honourable motives, however often mixed with vested interests and lack solid experience in scientific experimentation and the interpretation of scientific results) instead of listening to those who have spent their scientific career on the subject and are wrongly blamed to work in the interest of industry.

Food security - sufficient nutritious food for all at all times to live a productive life - is one of the basic human rights, which is to date beyond reach for more than one billion. Of course there are numerous causes. But there is no doubt that mankind has no choice but to produce more food on less agricultural land, with reduced input in fertilizer, agrochemicals, water, and energy. This problem can by no means be achieved, as often proposed in Europe, by focusing on 'organic farming'. Contrary to numerous claims by the organic lobby, organic farming has advantages with regards to soil fertility, but is suboptimal for productivity (one reason for financial substitution in Europe). To produce sufficient food world-wide for a growing population, will require a massive investment in a wise combination of all available know-how and technology (as e.g. exemplified by the strategy of 'integrated production'), and substantially increased financial support for agriculture and agricultural research on all aspects of agriculture (including organic).

To block - as done in Europe - one of the most potent technology (GM technology) for ideological reasons, at a time when it is clearly visible that only a concerted effort of all proven technologies can help to secure food production, against decreasing agricultural resources, expected negative effects of climate change, and increasing competition from wasting agricultural products in 'biofuel' production, is definitely very bad governance. This may not be immediately obvious in Europe which maintains a highly subsidised, artificial agriculture system and supports 'luxurious' organic production on the basis of import from food stuff from countries where millions starve to death (and where unproductive organic farming is the consequence of poverty and not of life style choice). As the European GMO attitude has a pronounced effect on the decision makers in developing countries which can not afford such a choice because use or non-use of GM technology is a question of life or death of millions of poor, European decision makers carry a heavy moral responsibility to base their view on scientifically sound facts instead of ideology-based opinions.

It is, therefore a must for every responsible politician in Europe involved in decisions about novel technology and agriculture, to read the Hungarian White Paper. I would also recommend in this context to study the 'Statement' published by the Pontifical Academy of Sciences. For those who have not even one minute now I offer the following 'telegram' summary: GM technology has a proven record of being the safest possible technology for the improvement of crop plants. In contrast to decades of claims by GMO-opponents there is, from all the large-scale application by millions of poor farmers, from 25 years of specific and published biosafety research, and from all regulatory oversight, not a single documented case of harm world-wide to any environment or any consumer. The technology has led in addition to well documented benefits to developing countries, the poor and the environment despite having been applied, so far, exclusively for commercial interests of a few large agbiotech industries. The cause for a de facto monopoly of the technology is not to be blamed on the technology, but on the established GMO-regulations, which effectively prevent that the technology can be used by public institutions for public good. Those reading the Hungarian White Paper and the Statement of the Pontifical Academy of Sciences will realise that there is an urgent need to change public attitude and regulation.

\section{Reference:}

Transgenic Plants for Food Security in the Context of Development. Proceedings of a Study Week of the Pontifical Academy of Sciences. Editors: Ingo Potrykus \& Klaus Ammann. NewBiotechnology, vol. 27 (5), 30 November 2010, pp. 443-717. Available as 'open source' publication via internet under the Vatican homepage www.vatican.va/roman_curia/pontifical_academies/cdscien/ 2010/newbiotechnologynov2010.pdf. To avoid any misunderstanding: this is not an official statement of the Curia or the Academy, but of the participants of the study week. 


\section{Editor's introduction}

\section{ERVIN BALÁZS, DÉNES DUDITS, LÁSZLÓ SÁG}

A critical issue of humanity's future is creating the conditions of sustainable development. Efforts to devaluate the role of science and to incite groundless fear of science-based technical development are wrong and cause much damage. At the beginning of the industrial revolution, the weavers of Lyon destroyed their looms for fear of loosing their jobs. The machine-breaking Luddite movement has become a historic symbol. Anarchistic civil movements, the machine breakers of our time destroy the experimental plots of green biotechnologists. What they do is primarily political fight directed against globalization and multinational companies, and they do not care about the purpose and potentially useful properties of the breeding material, "the plants of the future" growing in the damaged plots. As people gain in knowledge, sooner or later they accept, nearly without reservation, the fruits of technical development provided by science. An often cited example is the decree issued in England in the $18^{\text {th }}$ century, at the time of the introduction of the steam locomotive: a rider waving a flag had to ride ahead and signal the arrival (at a velocity of $4 \mathrm{~km} /$ hour) of the dangerous monster. It is not probable that any horseback rider would dare to signal the danger of present-day trains rushing at over $300 \mathrm{~km} /$ hour.

The agriculture and, first of all, the farmers themselves of our age are faced by an inescapable social expectation, that of having to produce more high-quality food on increasingly smaller areas of arable land. Yield safety is threatened by the growing scarcity of water and by the increasing frequency of extreme climatic events due to the climate change. Undoubtedly, long-term sustainable agricultural activity can only be pursued in close harmony with the environment. This requirement can only be met by a professional agricultural sector that utilizes the results of research and development and produces food and raw materials for the population of the Earth by integrating state-of-the-art technologies. It is becoming more and more obvious that we are on the threshold of a food price explosion with unforeseeable social consequences. It would be naivety to suggest that gene technology can offer a solution for all problems, but it can certainly contribute to the worthy cause of improving the quality of life of an increasing number of people by providing better plant cultivars and technologies. As the slogan of the Agricultural Organization of the United Nations puts it with beautiful simplicity: "Fiat panem - Bread for all".

This book reflects the scientific standpoint of those representatives of Hungarian science, who - unlike the scare-mongers of political movements consider gene technology a method serving the improvement/breeding of microorganisms, plants and animals, and firmly believe that organisms with improved properties contribute to the welfare of humanity. The editors take this opportunity to express their gratitude to the authors of the individual chapters for sharing their highly professional, scientifically dedicated thoughts.
We thank the Barabás Zoltán Federation of Biotechnology and the Pannonian Plant Biotechnology Association for funding the publication of this book. Preparation of the volume for the press is hallmarked by the excellent and conscientious work of Zsuzsa Keczán, Klára Godó and Mária Tóth. Special thanks are due to the translators, our colleagues Erzsébet Fejes and Anett Bacskovszky.

11 March, Szeged 


\section{Statement on Genetically Modified Organisms from the Section of Agricultural Sciences of the Hungarian Academy of Sciences}

In view of the ever widening role of biotechnology, particularly of gene technology and genomics in plant breeding, plant protection, the bioenergy industry and the manufacture of basic pharmaceutical products, the Section of Agricultural Sciences of the Hungarian Academy of Sciences formulated the following statement at its session of 26 May, 2010:

1. Judging by the evidence of recent global scientific and economic achievements, gene technology plays an increasingly decisive role in agricultural innovation and in the establishment of new technologies.

2. The methods of gene technology and genomics can more efficiently ensure the future competitiveness of Hungarian agriculture and hence, of plant breeding.

3. The role of eco-friendly agrotechnologies can be enhanced by the application of biotechnology in general and of gene technology in particular.

4. The medical, environmental and soil safety of the new gene technological products and the prevalence of long-term economic goals should be warranted by using scientific methods, in accordance with internationally accepted standards.

5. Scientific evidences should be granted a determining role in the public debate on genetically modified (GM) crops.

6. It is recommended that the decisions of the Hungariangovernment and legislation on gene technology be periodically reviewed in the light of new scientific results.

7. The competitiveness of Hungarian agriculture would be served the best by regulations granting equal opportunities for plant breeders as well as for farmers in a free choice of technologies.

8. For the best interest of agroinnovation, the capacity, funding and competitiveness of agrobiotechnological education and research should be increased.

9. The public acceptance of gene technology should be promoted by dissemination of scientific knowledge for the general public.

\section{Detailed justification}

Biotechnology applies the results of the basic science of biology in technological developments. Genomics is a new, dynamically advancing field of genetics that researches the function of the genomes of living organisms, the interaction and networking of genes. Gene technology is a recently rapidly expanding area of biotechnology that uses the methodology of molecular genetics and genomics.
Gene technological methods have made possible the generation of genetically modified living organisms both for research and for practical utilization.

The societal reception of genetically modified (GM) organisms and the products derived thereof is very contradictory all over the world, and the same is true in Hungary. Studies and statements on this subject, authored by renowned and opinion-shaping experts and published in the international and national printed and electronic media often contradict each other, confusing laymen rather than helping them make up their minds about the issue.

The opinion of the general public, especially that of the citizens of the European Union is significantly different regarding the medical/health care applications and the agricultural utilization of modern gene technological procedures. People are typically tolerant towards the utilization of GM microbes and animals for the production of medicinal preparations and for use as experimental subjects in biomedical experiments, whereas opinions are highly divided on GM plants and the utilization of gene technology in food production. Attitudes can be quite extreme, ranging from complete rejection to unconditional/ restricted utilization.

The introduction of GM plants to cultivation has also provoked a far-reaching debate in Hungary. In our opinion this is basically correct and desirable. It is regrettable, however, that occasionally, - in addition to scientific arguments pro and contra, - excessive, personal and provocative manifestations also occur. The Committee of Agricultural Biotechnology therefore agreed that the time was ripe for taking a stance, based solely on scientific arguments, in the GMO debate.

A genetically modified, in other words transgenic organism is the carrier of an artificial DNA construct synthesized by gene technological methods, which was artificially integrated into the hereditary material (genome) of the organism. These gene constructs induce the production of new proteins in the target cells, or modify the functioning of the original genes of the organism in some way. This modification is unique: each individual transgenic event will have a different effect on the properties of the given organism and its progeny. GM organisms can be classified as genetically modified microbes, animals and plants.

GM microbes (bacteria, viruses, fungi)

There is a long-standing tradition of the biotechnological utilization of microbes in the food processing industry (e.g. beer brewing, dairy industry). Gene technological development of these technologies is one of the intensively studied fields of biotechnology. The cheap and safe mass production of human proteins of medical significance (e.g. insulin, blood clotting factors, growth hormone etc.) has been made possible by the use of GM bacteria. Due to its safety, the utilization of GM bacteria for such purposes has gained tremendous importance in the last few decades, because the earlier technology based on extraction of proteins from cadavers involved increased risks of the contraction of AIDS, hepatitis, Creutzfeldt-Jakob disease and other infectious diseases. 


\section{GM animals}

In the case of GM animals, the aim of the artificial introduction of synthetic gene constructs is again the induction of the production of certain proteins in the target cells. In addition to experimental use for basic scientific purposes, in the past few years transgenic domestic animals (e.g. sheep, goat, cattle, chicken etc.) have also been generated for human therapeutic purposes. The animals obtained excrete, into their milk or egg-white, proteins used for medicinal purposes in humans, originally not produced in the respective animal species (e.g. tissue plasminogen activator, $\alpha 1$-antitrypsin, $\alpha$-lactalbumin, $\alpha$-glucosidase etc.). Similarly useful are animal model experiments using transgenic technology that provide often unique possibilities for studying the development and therapy of various diseases. These methods are expected to gain outstanding significance in human health care in the near future.

\section{GM plants}

New traits introducible into plants by gene technology include enhancement of resistance to herbicides, pathogens and pests, improvement of stress tolerance and increasing the nutritional value of edible plant parts. The first GM plants were commercialized in 1994 and their adoption and significance have steadily increased ever since.

The societal judgement and acceptance of GM plants are significantly affected by the relevant biological knowledge of the potential consumers, which can be expanded by the dissemination of appropriate, objective information. The development of the fields of science involved rapidly outdistances the growth of the relevant knowledge of the general public. The "biotechnological gap" keeps widening, and science and its practical utilization are increasingly alienated from the opinions and acceptance of the general public. It is therefore high time to start a wide-ranging information campaign on GM crops with the aim of supplying all the missing information required and needed by consumers, in a plain, easily comprehensible form. This information has to be objective and has to cover the questions frequently asked by consumers about gene technology, such as the ecological and food safety risks of GM plant production and the achievable economic and consumer benefits of these plants. We also think it is important to improve the knowledge of the general public on the present limitations and future possibilities of GM technology.

Today in Hungary it is theoretically possible to grow, on condition of compliance with the pertinent rules, the GM crop varieties approved in the European Union. In 2005, however, Hungary (just like several other EU member states) invoked the safeguard clause against the only de facto producible GM maize variety, temporarily prohibiting its production. We emphasize that the debate presently ongoing in the media is about the cultivation of a single GM maize line (MON810) in Hungary. The general assessment of GM crops must not depend on the possibly unfavourable properties or, maybe, unreasonable introduction of this one hybrid.
By right of our membership in the EU, a wide range of GM plant varieties will soon be available for Hungarian farmers. Each of these carries a different genetic modification, and their risks and benefits can only be evaluated on an individual basis.

Most of the participants of the GMO debate agree in that the large-scale production of GM crops has to be preceded by detailed, objective and careful risk assessment and by control studies on environmental and human health issues. However, there is no agreement whatsoever regarding the adequacy of such studies in progress, which come under the competence of the appropriate institution of the European Union (EFSA). We support the notion that the long-term effects of GM crops on the environment and on human health should be investigated on the basis of a compulsory protocol. The institutional background and the necessary regulations should be provided by the government, whereas the expenses of these investigations should be covered by the variety owners, with appropriate safeguards of the independence of the investigations in place.

Regarding the cultivation of GM crops, we think it important that GM crop production in Hungary should only be possible under strictly controlled conditions without, however, making impossible the economic and reasonable cultivation of approved GM varieties.

In addition to the application of the above principles in Hungary, they should also be advocated at the appropriate EU forums, in front of the responsible committees as well as in the European Parliament.

The experts produce numerous arguments both in favour and against the cultivation of GM crops. It is under debate whether or not the food produced from GM plants that are cheaper to grow, more delicious to eat or more healthy (e.g. have a higher vitamin content) can help solve the global problems of feeding humanity.

A fact not to be neglected is, however, that according to the latest report of the International Service for the Acquisition of Agri-Biotech Applications (ISAAA), in 2008 the global cultivation area of GM plants was more than 125 million hectares (an increase of nearly 11 million hectares in a year). The EU has already approved several dozens of GM varieties, and applications for the approval of several hundred more are now being processed. The present legislation of the EU does not allow to maintain the GMO-free status of Hungary, not even in the middle term. The next, more developed generations of GM plants are already being shaped on the benches of research laboratories all over the world. 13.3 million farmers are estimated to grow GM plants in 25 countries. Thus, GM plant cultivation technology is rapidly spreading and developing, and this trend will most certainly continue in the near future.

If we do not want our Hungarian research and breeding institutes to stand on the sidelines in a decade or two and watch their science pass them by, now is the time to make increased efforts to provide funding for this type of research. This will enable Hungarian researchers to keep up with new knowledge and technologies and will allow Hungarian scientific laboratories to retain their competitiveness in this highly competitive branch of applied research. 
Based on the tendencies observed globally, the Hungarian general public has to be prepared to be ready to accept those GM crops whose safe cultivation and consumption have been verified by scientific methods beyond any doubt.

\section{Position of gene technology amongst genetic modification tools}

\section{PÁL VENETIANER}

The scientific discovery that has made agricultural gene technology possible is a simple biochemical procedure, whose more adequate designation is "in vitro DNA recombination". It was heralded by an experiment performed by Paul Berg and co-workers (carried out in 1972 and later rewarded by the Nobel prize), in which the complete DNAs of two different organisms (a bacteriophage and an animal virus) were joined for the first time to produce a functional product. DNA recombination was developed to a generally applicable technique by the experiments of Stanley Cohen, Herbert Boyer and their co-workers published in 1973. These authors showed that DNA can be cut at well-defined points with the help of restriction endonucleases discovered a few years earlier, and joined using other enzymes, making it possible to join or rearrange DNA fragments of different origins just like the cutting of magnetic tape, film or videotape, and that the products may retain their functionality after introduction into living cells. The enormous potential of the new technique immediately provoked a heated debate both within the scientific community and in the wider social-political scene, at first nearly exclusively in the USA. At the time the debate was mostly about microbiological applications and ebbed out within a few years, after the publication of the first, spectacularly practical and useful results (production of an important human hormone by a genetically engineered bacterium) and numerous experiments demonstrating the safety of the new technology. In 1982, however, functional foreign DNA was first introduced into animals and later also into plants. As a result of this success, in 1994 the first "transgenic" plant was introduced into commercial production, and the somewhat misleading term GMO (genetically modified organism) was coined for the designation of this type of plants, animals and microorganisms. This designation is inaccurate, because, obviously, traditional breeding also genetically modifies its objects; what is more, it is also correct to claim that every human being is the product of the genetic modification of the mother's genetic material by that of the father. (Even worse is "génkezelt" (literally: "gene-treated"), a term ineradicably spread in Hungarian media, suggesting that the plant in question was immersed in a gene solution or that genes were introduced into it by smearing with a brush.) As a matter of fact, GMO means an organism into whose hereditary material a DNA fragment was introduced in a planned, targeted fashion, circumventing natural crossing. If the DNA introduced originates from a different species, the resulting plant or animal is called "transgenic", whereas if the transferred DNA comes from the same species, the product is a "cisgenic" organism.

Thus, the year 1994 saw the kick-off of a new era, that of applied agricultural gene technology. For some years its development was relatively uneventful; in 1998, however, a tremendous upheaval broke out, mainly in Europe, in the wake 
of Árpád Pusztai's interview for the BBC about the alleged risks of agricultural gene technology, which has continued to this day. Objective deliberation of the issue is made difficult by the fact that the global opponents of the technology rarely make it clear which of the following possible targets they are aiming at:

1. The technology per se (i.e. do they consider any GMO dangerous in itself?)

2. The phenotype of certain GMOs (i.e. the concrete traits introduced by the technology such as herbicide tolerance and pest tolerance)

3. The societal (economic, political, legal [patent law]) problems of the applications of agricultural gene technology

The reason why this distinction is necessary is that, out of the three targets, it is only the first one that relates specifically and exclusively to gene technology, since, for example, herbicide-tolerant plants also exist in nature and can also be developed by traditional breeding, and the concomitant social phenomena mentioned are quite obviously present in our world even without gene technology. Thus, the scope of this chapter will be limited to the technology itself, irrespective of the phenotype of the GMOs produced or the concomitant social phenomena. The first question to decide is whether or not gene technology is a revolutionary new method, fundamentally different from traditional breeding. Instead of starting a debate on the definition of "revolutionary new", let us try to review the differences and the similarities of the two procedures.

There is no difference in that

- ...both procedures are artificial rather than natural;

- ...both can create a construct not occurring in nature;

- ...both can cross species boundaries;

- ...both by-pass the "filter" of evolution;

- ...both can produce toxic plants harmful for the consumer;

- ...the plant produced by either procedure may harm the environment/ ecosystem or

- ...reduce biodiversity.

Each and all of the above listed statements are periodically raised as accusations against gene technology, but are, obviously, equally true for a number of the procedures and products of traditional breeding.

The only fundamental differences are that

- ... in the course of traditional breeding, tens of thousands of genes are randomly mixed with tens of thousands of genes in every case, whereas gene technology introduces a single known gene (or sometimes a few genes) in a carefully planned fashion;

- ...the gene to be introduced may come from any living species, or may even be a synthetic construct.

As regards the first item, i.e. the planned and targeted character of the intervention, this would be difficult to attack with rational arguments; the opponents mostly question its truthfulness (i.e. whether the intervention is indeed planned and targeted ) - I will come back to this point later.
The second item is the one that that attracts the largest number of attacks from the opponents (especially among the general public), although it has to be noted that (i) there also exist GMOs into which genes from the same species were introduced: these are the so-called cisgenic GMOs, such as the first GMO food ever commercialized, Flavr-Savr tomatoes; and (ii) the introduction of a "foreign" gene circumventing normal reproduction (the so-called horizontal gene transfer) most certainly exists in nature, although is it extremely rare.

Since the method for creating GMOs is described in the next chapter of this book, I will now limit my discourse to the question whether gene technology per se has any hazard irrespective of the quality of the trait introduced, and I will attempt to critically analyze the most frequent objections concerning the technology itself.

\section{Is it potentially hazardous that the DNA introduced is "foreign"?}

No, because the structure of DNA and the genetic code are uniform in all living organisms, and the genes are "portable". It is true that the distribution of equivalent (synonymous) codons may be different in each species, however this may only affect the level of gene expression at the most, and not the structure of the gene product. Besides, in numerous applications the codon usage of the foreign gene is modified to fit that of the host plant. It also has to be noted that not a single atom of the "foreign" gene is physically derived from the donor species, only its information content is transferred. And, of course, we all have to realize that the DNA humans consume in their food is exclusively foreign (cannibals being the only exception), and comes from a large variety of species, obviously without doing anyone any harm.

Can the regulatory regions of the genes introduced, for example the so-called $35 \mathrm{~S}$ promoter, a strong promoter derived from a plant virus (promoter: a regulatory element situated at the beginning of the gene, responsible for the intensity of gene expression) represent a potential hazard?

No, because this promoter is present in a number of plants widely consumed by humans and is known to function very poorly - if at all - in animal (and human) cells. Also, our food contains many promoters capable of functioning much more efficiently in human cells, and, naturally, these do not represent any hazard at all.

Is it true that the insertion of the foreign gene is random and impossible to direct?

Yes, it is, but the danger is only for the researcher working on the development of the particular transgenic plant, because the efficiency of his/ her work is reduced. If the insertion of the foreign gene ruins or weakens an important host gene (this is theoretically possible), this cell will loose its viability. 
In the course of the development the researcher must make sure that the cell into which the gene was inserted and the plant grown therefrom is healthy and intact in every respect, except for the presence of the trait encoded by the foreign gene. Lately the DNA sequence in the vicinity of the inserted gene is routinely determined in order to confirm that no functional host genes were damaged.

Can the insertion of a foreign gene cause other unplanned, uncontrollable changes in the host genome?

Theoretically it can; there are some reports describing the loss of small DNA segments from the genomes of certain GM plants. It is known, however, that rearrangements of this type may also occur and indeed do occur at quite high frequencies in the course of normal crosses. The so-called "jumping genes" capable of spontaneously changing their position within the genome were discovered in the middle of the last century by Barbara McClintock just in maize, one of the most important objects of gene manipulations. It is the consequence of spontaneous rearrangements of this type that there may be far larger differences among the genome structures (DNA sequences) of various, traditionally bred maize varieties than between a GM maize variety and its non-GM progenitor. There are no data to support the assumption that gene manipulation might increase the number or the extent of these rearrangements.

\section{Can the product of the foreign gene cause other unplanned changes in the cells of the host plant, such as the appearance of a harmful metabolic} product, or a reduction in the amount of important nutrients?

Theoretically it can. Although the proteins encoded by the transgenes of known GM plants do not participate in plant metabolism and have no known interactions with other proteins, the existence of so far unknown interactions cannot be excluded at all. It can be considered as certain, however, that the probability of such unplanned and unpredictable interactions is by several orders of magnitude higher in the case of traditional breeding accompanied by the rearrangement of thousands of genes and gene products than in the case of the single gene and gene product transferred by gene technology. This banal statement is naturally confirmed by numerous experimental data. Analysis of the total protein and low-molecular-weight metabolite content of a number of GMOs has shown that the difference between the GM variety and its non-GM progenitor is considerably smaller than the differences between certain varieties bred by conventional methods. Naturally, the compulsory tests required by the approval procedure of each GM variety also attest that the difference between the GM variety tested and its non-GM progenitor regarding nutritionally important cell components and the concentration of harmful or toxic materials possibly present in the plant cell does not exceed the differences among certain traditionally bred varieties.
So far I have dealt with the alleged hazards of the application of recombinant DNA technology. It is important to add that researchers have recently developed techniques much more worthy of the designation of "gene modification" than those described above, even though the products of these techniques (which have not yet been commercialized) cannot be regarded as GMOs according to the presently valid regulations. The reason for this is that these new techniques do not introduce foreign genes into plants, but use various DNA manipulation methods instead to bring about mutations, to silence or excise genes or to modify regulatory regions etc. in a precisely planned fashion and at well-defined locations in the genome of the host plant, i.e. "genetic engineering" in the strict sense of the word is performed without using DNA from a different species. The practical results of these technologies and the debates provoked by them are still ahead of us

\section{Summary}

At the end of this chapter let me quote from the closing document of the Conference of the Pontifical Academy held in the Vatican in May 2009. "The genetic improvement of crop and ornamental plants represents a long and seamless continuum of progressively more precise and predictable techniques. As the U.S. National Research Council concluded in a 1989 report: 'As the molecular methods are more specific, users of these methods will be more certain about the traits they introduce into the plants and hence less liable to produce untoward effects than other methods of plant breeding." 


\section{Procedures for the generation of genetically modified organisms}

\author{
LÁSZLÓ SÁGI, ELEN GÓCZA, KORNÉL KOVÁCS
}

\section{Introduction}

Life scientists often believe that communicating technical details on their subject - in this case the production of genetically modified (GM) living organisms - is so interesting in itself that it will inevitably convince a reluctant or ignorant public, and as a result, people will be happy to convert to their 'discoveries'.

That this notion has been a failure and wishful thinking rather than reality can be best demonstrated by the present anti-GM attitude in a number of EU-countries as well as by a strong 'no' to cultivation, processing and sale of GM crops. Hungary (and some of the neighbouring countries) is no exception to this type of public reaction, which is partially due to the not-so-neutral presentation of GM issues in national and local media (see also Chapter 17).

While dissemination of scientific information on the GM subject may not have been very successful in the popular media, the provision of comprehensible and unbiased information to people showing an interest in the issue as well as to decision makers is definitely justified. We do not want to be blamed for concealing the "obscure machinations" of scientists from the public.

In this chapter, we will therefore give a concise overview on (i) the major techniques currently employed for the generation of GM organisms (microbes, plants and animals), and (ii) the principles and practice on how to make these techniques ever more efficient and, at the same time, acceptable for the public.

Definition: transgenic or genetically modified living organisms are organisms each of whose cells stably contains the inserted DNA and this transgene is (or these transgenes are) transmitted to the offspring as well.

\section{Generation of GM microbes}

As scientists analysing basic cellular processes, they often extract enzymes or DNA from whole cells and study their activity and structure outside the cell. When a process is investigated in vitro, it can often be manipulated in ways that would be impossible if it was still taking place inside a cell. Natural processes resulting in the transfer of DNA from one cell to another cell (transformation, transduction, conjugation) have been discovered. A by-product of the quest for knowledge was the accumulation of a versatile tool kit for manipulating and analyzing DNA and proteins. Biological knowledge and biotechnology know-how have increased. Modern biotechnologies have harnessed enzymes and procedures that scientists copied and modified from nature.
The explosion of DNA technologies has in part been the result of the intense research focus on understanding the function of DNA from the beginning of the 1950s. One of the challenges facing the newly born molecular biology was the fact that in nature many genes are found on one DNA molecule. In the 1960s, scientists studying how certain bacteria resist infection by bacteriophages observed that the DNA of the infecting virus was cut into pieces at well-defined sequence positions: the corresponding enzymes were named restriction endonucleases or restriction enzymes (Figure 5.1.). By now more than 5,000 restriction enzymes have been identified and isolated from hundreds of bacterial species.

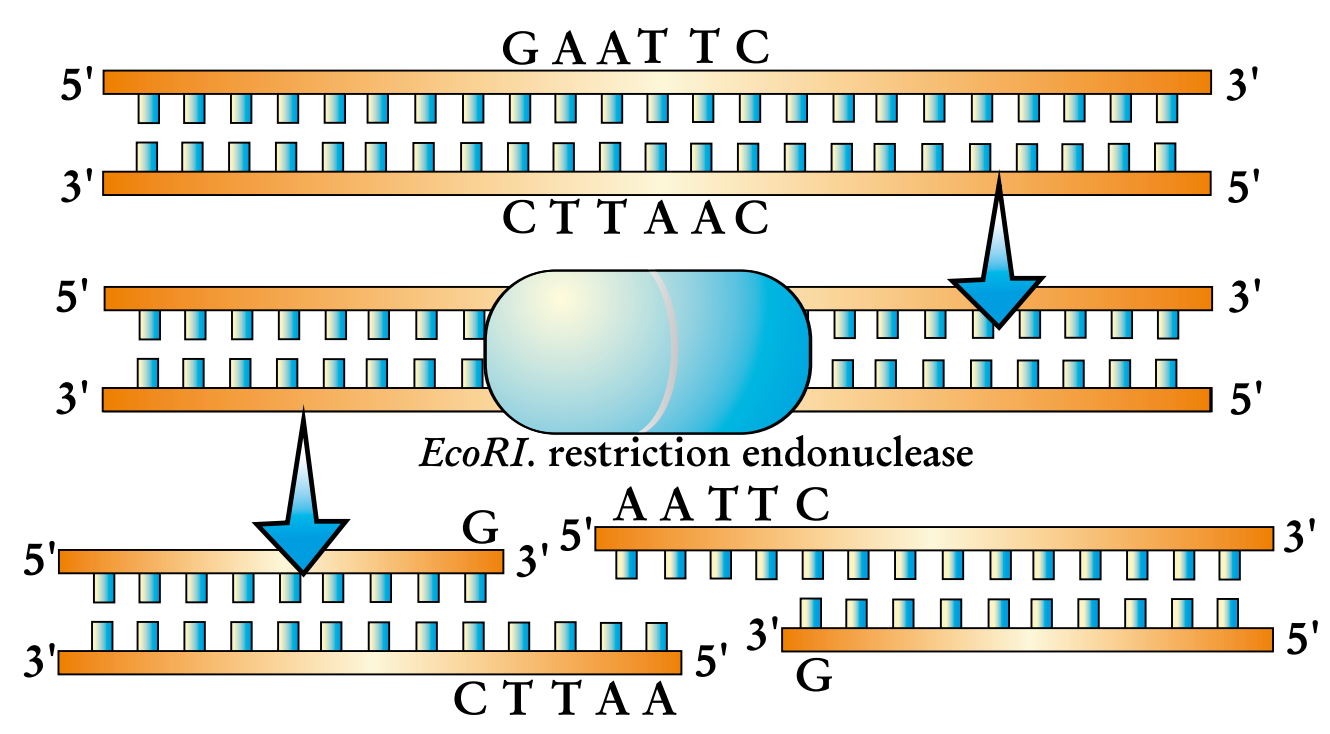

Figure 5.1. Schematic activity of a restriction enzyme from the $E$. coli bacterium

These enzymes are used like precise DNA scissors to cut DNA molecules into precise pieces, which can then be used either for identification or for making copies of desired genes. Identification of specific DNA sequences is useful in a number of courtroom cases such as the identification of criminal suspects by their DNA pattern, verification of biological fathers when in doubt, or even for clarifying food safety issues. Genes that carry traits, which we decide to exploit, are then multiplied and their gene products can subsequently be generated at will. The polymerase chain reaction(PCR) is now used in many kinds of applications from disease diagnosis to studying ancient DNA. PCR can produce over 1 billion copies of a DNA segment from a single starting molecule in about 1 hour of amplification. It is a molecular copy-machine working at amazing speed. The term cloning means the production of identical copies of something. For instance, cars rolling off the assembly lines are clones. In biology, cloning specifically refers to the production of genetically identical copies. When applied 
to DNA, cloning is usually understood as the transfer of a piece of DNA into a cell in such a way that the information encoded in the DNA piece will be exactly replicated and maintained in the recipient cell. There are many technical solutions to clone DNA, which usually involves a recombinant molecule using a carrier referred to a "vector". The vector facilitates the transfer of DNA into the new host cell and its maintenance within that cell. Today many kinds of cloning vectors are available, tailored to various kinds of host cells and applications. The most common vector is a plasmid, which is a small, circular DNA molecule that can copy (replicate) itself. Once recombinant DNA has been introduced by a plasmid into a batch of host cells, the next task is to identify the cells that took up the recombinant DNA. Transfer of genetic information is inherently inefficient; therefore marker genes are often used to aid selection of cells that successfully received the recombinant DNA. The most common marker genes encode resistance to antibiotics. This generates the principal risk of transferring antibiotic resistance to pathogenic strains in nature. In order to exclude this possibility, industrial GMO strains are created by insertion of the desired gene(s) directly into the chromosome of the target microorganisms, thereby eliminating the need for selection based on antibiotic resistance.

Genetic modification takes the process one step further in that it introduces a specific gene or genes into a bacterial host so that the proteins encoded by the foreign genes will be expressed. Recombinant DNA technology is a generic term that encompasses a number of experimental methods leading to the transfer of genetic information from one organism to the other. The first step in this process is cloning DNA fragments that encode a gene of interest. If this gene is from an eukaryotic organism to be expressed by a bacterial host, one should take into consideration a major difference between eukaryotes and prokaryotes: most eukaryotic genes have introns, i.e., DNA sequences that are spliced out following transcription in an eukaryotic cell. It is also necessary to add built-in regulatory signals, such as a prokaryotic promoter, so that the gene can be correctly transcribed and expressed in the new host. If the new gene is combined with such molecular devices it will be expressed and the corresponding function will be manifested in the bacterial host, e.g. by secreting a protein in the growth medium.

The fact that microorganisms can produce food additives and other valuable substances is nothing new. Genetic modification, however, has made it possible to engineer bacteria and fungi for large-scale production of substances at an economic advantage compared to conventional, industrial methods. Additives, amino acids, vitamins, flavours, enzymes - countless substances used in food production are produced by GM microorganisms. In many ways, these biotechnological methods have replaced chemical, synthetic production.

The advantages are obvious: microorganisms grow rapidly and in most cases are easy to cultivate. The fact that they 'work' under normal conditions means that (unlike conventional production methods) they do not need high temperature and pressure, or harsh chemicals. Using microorganisms is much more environmentally friendly than traditional chemical-synthetic methods; they require less energy and make use of renewable resources. Production leftovers are easily biodegradable and have minimal impact on waste water. In order to biotechnologically produce a certain substance, microorganisms must be identified that produce the substance naturally. There are many bacterial and fungal species that are known to produce valuable compounds. However, it was not until genetic modification came about that these biological methods became economically viable. Targeted genetic modification has not only enhanced the productivity of these methods, it also has resulted in the production of substances which was previously impossible. Genetic modification has dramatically expanded the potential of biotechnological methods.

The production of substances with the help of microorganisms takes place in fermentors. These closed tanks are usually made of stainless steel and are used to set up optimal conditions so that microorganisms can thrive and produce the desired product in large quantities. When the growth and production phase ends, the substance of interest is extracted and purified. No traces of microorganisms are left in the final product, and no microbial DNA is detectable.

\section{Generation of GM plants}

Let us start with pointing to the strikingly similar mechanisms nature developed and science adapted for higher plants to maintain or increase genetic variation by the transfer and (re-)combination of their genes.

In nature, the germinating pollen bores in the pistil a channel - similarly to the tunnel of the underground - to the vicinity of the egg cell, which is naturally receptive to exogenous DNA (Figure 5.2.). Along this channel, a few picograms (one trillionth of a gram) of hereditary material, i.e. DNA are transported via the sperm cell into the egg. This DNA is distributed in the course of further divisions of the egg cell into self-replicating packages (the chromosomes) that each contain a few thousands of genes - DNA segments that encode proteins - out of several tens of thousands in total.

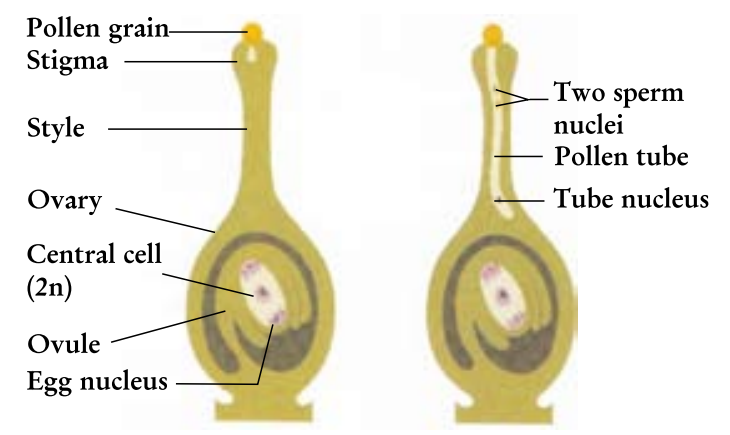

Figure 5.2. The delivery of hereditary material, DNA in plants via fertilization (source: www.biologyjunction.com/plant_reproduction.htm) 
Scientists do nothing substantially different during genetic modification. Since egg cells are deeply embedded in the pistil and are not easily accessible (Figure 5.2.), scientists employ the reversible developmental program (totipotency) of plants to generate de novo entire plants from many types of plant tissues, e.g. pieces of young leaves, under defined and controlled (in vitro) culture conditions. The most widespread application of this tissue culture technology is the mass multiplication of ornamental or herb plants: most of the plants sold in florist shops have been produced this way for years or even decades.

Next, scientists also create by various means an entry point in the plant cell for the DNA, so that it can enter through the cell wall and the underlying membrane. This point is - just as in nature - a micro-channel, which can be 'constructed' either by a microscopic bullet, a bacterial 'drilling platform' (Rhizobium), an electric impulse, or a very fine needle (microinjection), just to name a few alternatives. Thereafter, another self-replicating DNA package smaller than chromosomes, a plasmid is integrated into the plant DNA. Plasmids usually contain only a small number of well-defined genes and their length does not amount to even one millionth of the total DNA in a wheat cell.

From a technical point of view, two major components - besides routinely performed tissue culturing - of successful gene transfer in plants can be distilled from the processes described above:

- functional gene construct ('what to insert'),

- efficient transfer method ('how to insert').

In order to execute a function, a gene construct must have two components. The first one is a coding region that stores the information for the synthesis of transcribed or messenger RNA and eventually (though not always) for a translated protein molecule. The second component is a set of regulatory DNA sequences that are responsible for the start, execution and termination of RNA transcription from the coding region and for subsequent editing of the RNA transcript. These regulatory DNA elements (e.g. the promoter and terminator region) are usually located before the start codon (with the base sequence ATG) or after the stop codon (with base sequence TAA, TAG or TGA) of the coding region or are inserted in it (introns). The structure of a gene is analogous to a simple electric circuit: the coding region serves as the bulb with its function to deliver light, and the promoter represents the switch that turns on the bulb (Figure 5.3.).

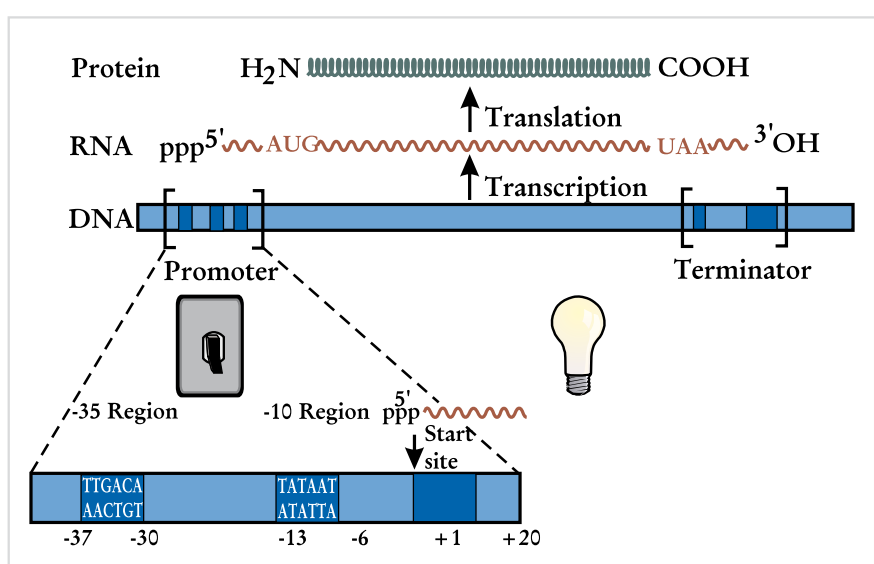

Figure 5.3. Schematic structure of a gene that consists of a region (working like a bulb) coding for RNA (and protein) as well as of regulatory signals (promoter and terminator) acting as a molecular switch. The promoter region contains conserved domains (dark blue stripes), which anchor DNAbinding proteins required for bending DNA into a functional spatial conformation

Certain 'reporter' genes are really able to trigger light emission in GM plants when connected to a suitable genetic switch. Spectacular examples are the luciferase gene from firefly (Figure 5.4A) and the green fluorescent protein (GFP) gene from jellyfish (Figure 5.4B), which are used for easy and sensitive detection of transgenic plant cells and tissues.

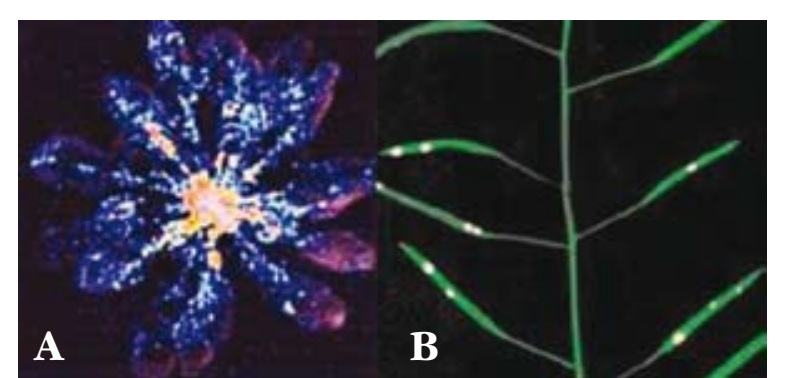

Figure 5.4. Bioluminescent and fluorescent reporter genes in GM plants. (A) Expression of firefly luciferase in the rosette stage of a young Arabidopsis plant (Lopez-Huertas et al., 2000, The EMBO Journal, 19: 6770); (B) pattern of green fluorescent protein expression in Arabidopsis capsules containing seeds (Stuitje et al., 2003, Plant Biotechnolog y Journal, 1: 301) 
Another widely used reporter gene is the GUS gene encoding the glucuronidase enzyme, which is detected by a characteristic indigo blue colour reaction (see Figure 5.6.) and which can be found in coli bacteria that exist in large quantities e.g. in the intestines.

\section{Gene transfer into plants}

During the three-decade past of GM plants a large number of gene transfer protocols have been developed or tested. These methods are usually distinguished as direct or indirect ones depending on whether the gene(s) to be transferred are introduced as pure DNA or by the assistance of a living organism. In this latter case, the intermediate organisms serve as biological Trojan horses to 'smuggle in' the desired genes. These vector organisms can be a number of bacterial species belonging to the Rhizobium genus or several plant viruses.

An ideal gene transfer method should fulfil a few essential criteria:

- versatility: simultaneous introduction of multiple genes on a single long or distinct DNA piece(s),

- simple integration pattern and stable gene expression in GM plants and their progenies

- controlled, site-specific integration (gene targeting),

- simplicity, low cost and efficiency,

- universality: applicability in (almost) any species

\section{Rhizobium-mediated gene transfer}

A few soil bacterium species of the bacterial family Rhizobiaceae (e.g. Rhizobium radiobacter, earlier: Agrobacterium tumefaciens) are famous natural genetic manipulators, as they are able to perform the unique feat of simultaneously transferring genes and proteins to plants (and in fact many other nucleated organisms), which is accomplished by a precisely engineered process that consists of five main steps (Figure 5.5.):

- (i) moving towards and attachment to wounded plant cells,

- (ii) activation of a two-component sensor system in bacterial cells to release transfer T-DNA,

- (iii) assembly of a 'chunnel' (pilus) between the bacterium and the plant cell,

- (iv) assembly and transfer of a DNA-protein complex into the plant cell,

- (v) transfer of this complex into the nucleus and DNA integration into the plant chromosome.

The genome of this bacterium consists - as a unique feature in nature - of four main elements, two types of chromosomes (circular and linear) and two circular plasmids. Interesting and, from our point of view, essential is the task division on one of the plasmids (Ti): the transferred (T-)DNA is separated from the virulence (vir) genes that orchestrate its synthesis, assembly and transfer
The 'purpose' of the above process for the bacterium is to colonize vulnerable plant cells and transform them into cellular 'factories' that produce carbon and nitrogen resources in the form of modified amino acids. To this end, genes are located on the transferred DNA segment (T-DNA), which encode specific enzymes to reprogram the hormonal metabolism of plant cells into proliferation and other enzymes to catalyze the biosynthesis of nutrients in the modified cells. As a result, large tumors called 'crown galls' are formed on the near- and underground part of colonised plants, which in turn poses a serious constrain in agriculture, especially in the ornamental sector and viticulture.

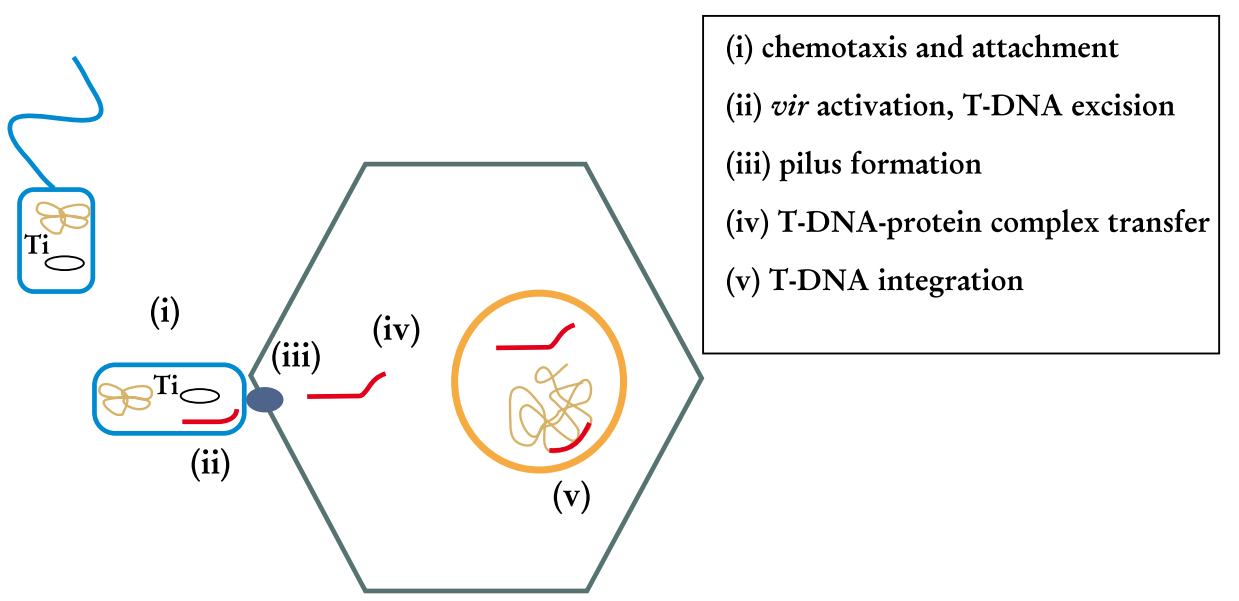

Figure 5.5. Major phases of the natural gene transfer process by Rhizobium. Blue cells correspond to motile (with a flagellum) or attached bacteria that contain chromosomal DNA and the circular tumor-inducing (Ti) plasmid. The green box depicts a plant cell comprising a nucleus (yellow circle) and genomic DNA, where the transferred bacterial T-DNA (red fragment) is transported through the 'chunnel' (blue spot) and integrated into (Pérez-Hernández and Sági, unpublished)

After a decennia-long intensive research of the Rhizobium enigma, scientists discovered how to put this natural process to the service of mankind. The key to this success was the recognition that the T-DNA sequence itself does not affect gene transfer, and it is sequences outside the T-DNA (such as the virulence genes) and flanking it (the so-called border regions) that are essential and instrumental in this process. This means that: (i) the hormone catalytic enzyme genes could be removed so that tumors are not formed any more, and (ii) the enzyme genes responsible for the biosynthesis of amino acid (opines) could be replaced by any other gene(s) that would still be transferred.

Technically, the transformation process itself is very simple; even a primary school pupil is able to execute it. It consists of dipping the plant tissue explants 
(e.g. a leaf piece) into a suspension containing culture medium and activated bacteria, then blotting the explants dry and co-cultivating them for 1-2 days so that gene transfer and integration can take place. The explants are then placed on antibiotics to kill bacteria that have done their job and are not needed any more; finally, transgenic cells are selected and regenerated in a few steps into GM plants.

Though a complex process from a biological aspect, the optimization of a few specific parameters also turns this method remarkably efficient in the laboratory. Some of these factors are composition of the medium for efficient virulence gene activation, the duration and temperature of incubation during plant-bacterium co-cultivation, and the bacterial strain/plant genotype combination.

The main advantages of the Rhizobium gene transfer are simplicity, low cost and efficiency in more than hundred plant species.

\section{Regulated gene expression}

A significant improvement in gene transfer technology is the more precise expression of transgenes. In first-generation GM plants, transgene expression was controlled by strong promoters, usually isolated from simple organisms, such as plant viruses. A major function of these organisms is fast and strong expression of their few genes in many types of plant cells, hence the corresponding promoters having sustained activity are called constitutive. However, this constitutive type of control is far from optimal for most transgenes in GM plants. For instance, a disease resistance gene against a leaf pathogenic fungus does not have to be strongly expressed in the seed if the pathogen is not able to infect seeds. Abundant expression may even be contra-indicated in certain scenarios, when for example an opportunistic pathogen would have a higher chance to 'uncover' a particular disease resistance mechanism and develop immunity against it. Also, expression of most native plant genes is finely regulated during development and thus for a successful transgene a similarly precise expression control is required.

To this end, plant scientists have been actively developing techniques for the identification and precise characterization of novel plant promoters and other regulatory sequences. As a result, a large collection of promoters is now available for tissue-specific and even cell type-specific expression of target genes in GM plants (Figure 5.6.). Some of these promoters are now being tested or already reached commercial application in next-generation GM products.

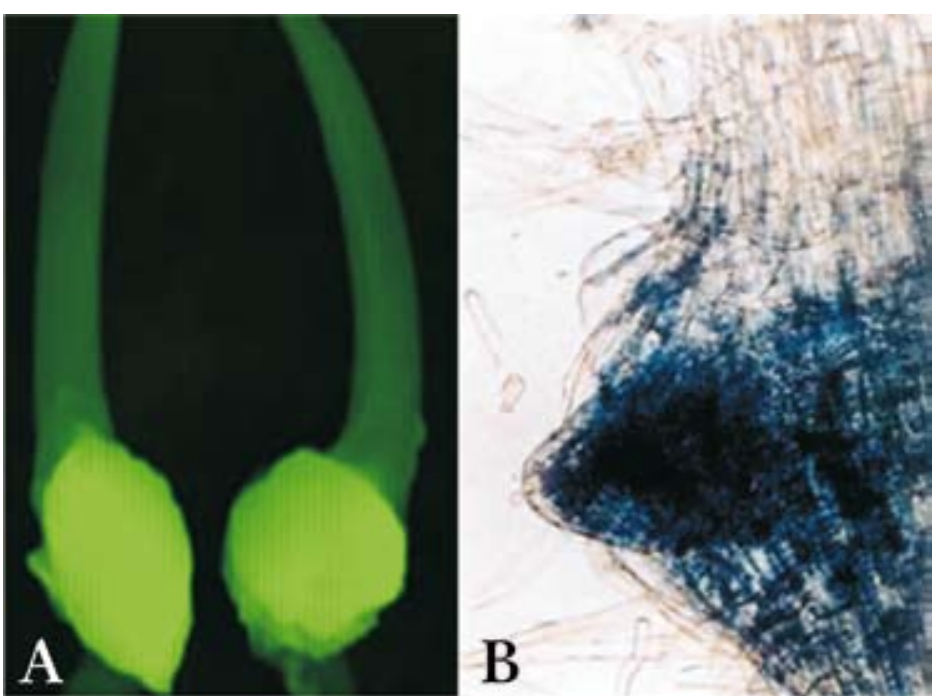

Figure 5.6. Transgene expression controlled by specific promoters in GM plants. (A) GFP expression in rice caryopses but not in germinating seedlings, (B) glucuronidase (GUS) expression in young root tip of banana (Schenk et al., 1999, Plant Molecular Biology, 39: 1221)

\section{Generation of GM animals}

The creation of genetically modified animals has enabled us to understand the functioning of numerous genes, and the generation of transgenic animals modelling human diseases has provided the opportunity to reveal the genetic background of certain diseases, resulting in the development of new medicines. The significance of the subject was acknowledged by the Medical Nobel Prize in 2007.

The creation of the first transgenic animals was made possible by the simultaneous improvement of several fields of science. In 1974, Rudolf Jaenisch injected a DNA virus into mouse embryos, which was the first successful genetic modification in animals (Proceedings of the National Academy of Sciences USA, 71: 1250). Gordon et al. announced in 1980 the generation of GM mice via the microinjection of recombinant DNA into the nuclei of single-cell embryos (Proceedings of the National Academy of Sciences USA, 77: 7380). By now, researchers have developed several DNA transfer procedures, including microinjection, electroporation, as well as application of viruses and artificial chromosomes (Table 5.1.). 


\begin{tabular}{|c|c|c|c|c|c|c|}
\hline Methodology & $\begin{array}{l}\text { Construct } \\
\text { type }\end{array}$ & Target cell & $\begin{array}{l}\text { Maximal } \\
\text { size of } \\
\text { construct }\end{array}$ & $\begin{array}{c}\text { Targeted } \\
\text { modification }\end{array}$ & Efficiency & $\begin{array}{l}\text { Technical } \\
\text { difficulties }\end{array}$ \\
\hline \multirow{5}{*}{$\begin{array}{l}\text { Micro- } \\
\text { injection }\end{array}$} & DNA & $\begin{array}{c}\text { nucleus of } \\
\text { zygote }\end{array}$ & $50-1000 \mathrm{~kb}$ & no & ++ & +++ \\
\hline & Virus & $\begin{array}{l}\text { perivitelline } \\
\text { space of zygote }\end{array}$ & $5-10 \mathrm{~kb}$ & no & +++ & ++ \\
\hline & Transposone & $\begin{array}{l}\text { cytoplasm of } \\
\text { zygote }\end{array}$ & $50-1000 \mathrm{~kb}$ & no & +++ & ++ \\
\hline & $\begin{array}{l}\text { Zinc finger } \\
\text { nuclease }\end{array}$ & $\begin{array}{l}\text { nucleus of } \\
\text { zygote }\end{array}$ & $\begin{array}{l}18 \text { bp target } \\
\text { sequency }\end{array}$ & possible & ++ & +++ \\
\hline & $\begin{array}{l}\text { Artificial } \\
\text { chromosome }\end{array}$ & $\begin{array}{c}\text { nucleus of } \\
\text { zygote }\end{array}$ & $100-2000 \mathrm{~kb}$ & no & + & ++++ \\
\hline \multirow{4}{*}{$\begin{array}{l}\text { Electro- } \\
\text { poration, } \\
\text { lipid } \\
\text { micelles }\end{array}$} & DNA & \multirow{4}{*}{$\begin{array}{l}\text { stem cell, } \\
\text { somatic cell }\end{array}$} & $100-2000 \mathrm{~kb}$ & \multirow{4}{*}{ possible } & +++ & \multirow{4}{*}{+} \\
\hline & Virus & & $5-10 \mathrm{~kb}$ & & +++ & \\
\hline & Transposone & & $50-1000 \mathrm{~kb}$ & & +++ & \\
\hline & DsRNA & & $19-23 \mathrm{~kb}$ & & ++ & \\
\hline
\end{tabular}

Table 5.1. Comparative description of the major techniques used to create GM animals

In most of these methods, the integration of DNA vector occurs mainly at random, which might disturb or suspend the functioning of a host gene if the transferred gene is inserted in the DNA of the host organism at that point. In addition, the introduced DNA may be inserted in an inactive chromosome region, making it functional only in a certain fraction of the cells, thus the GM animal generated is this way will have a mosaic expression pattern for the transgene. Targeted gene transfer can now be achieved through the application of special DNA vectors, utilizing homologous recombination and gene silencing, or by employing the ability of zinc finger nuclease enzymes to the controlled repair of DNA. This ensures that only the desired gene is modified or that none of the genes in the organisms carrying the transgene are changed.

\section{Procedures for the generation of GM animals}

DNA microinjection

The microinjection of DNA is the most common method for making transgenic animals. During the process, the DNA containing the transgene is introduced in the fertilized oocyte (zygote) by directly injecting the DNA dissolved in injection solution into the nucleus (Figure 5.7.), which requires special devices (e.g. micromanipulator) and highly skilled technicians. The efficiency of the method is relatively low, as only $5-10 \%$ of the animals born carry the introduced gene.

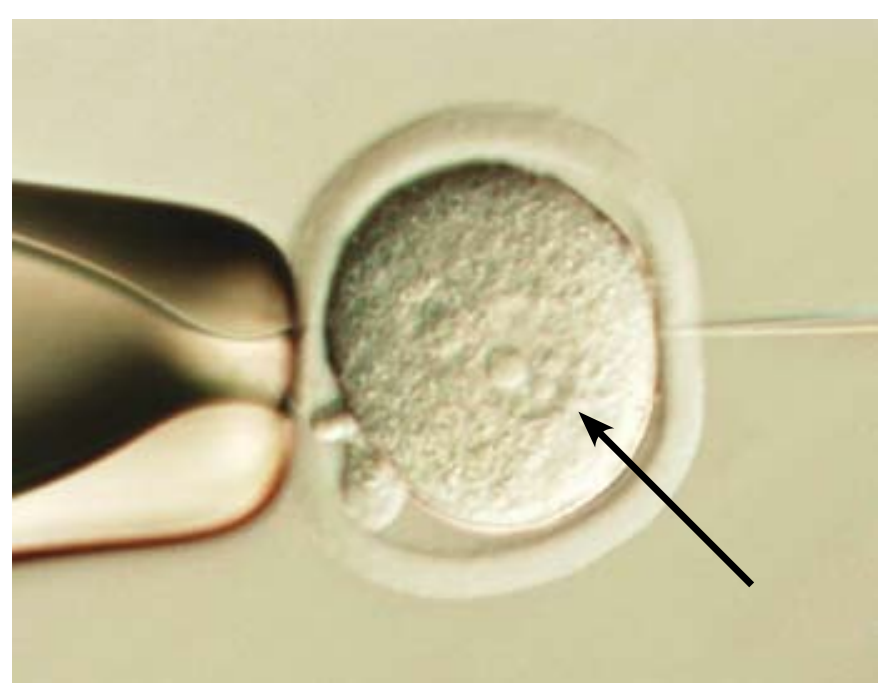

Figure 5.7. DNA microinjection into fertilized oocyte. The DNA is injected directly in the nucleus (indicated with an arrow) using a fine microcapillary pipette

The transgenic animals created by this method enable studying the effect of the protein produced on the basis of the introduced gene on the development of the embryos. In model animals, treatments suitable for curing certain diseases can be developed.

GM farm animals are generated for the modification of particular traits when the desired result cannot be achieved by traditional breeding methods, or it would take too much time. The experiments performed so far aimed at the alteration of endocrine glands, the structural proteins of milk and wool and the immune system as well as the development of disease resistance. Research on GM animals created for agricultural purposes has gained a number of promising results. The utilization of transgenic farm animals as bioreactors has also been put into practice (Chapter 8 ). 


\section{Knock-out and knock-in transgenic animals}

The first knock-out animals were created by targeted genetic modification. Homologous recombination ensures that the DNA vector containing certain elements of the target gene is integrated into the desired gene. In the event that the transgene is inserted in a gene sequence (exon) that is essential for the functioning of the target gene, the protein produced from this gene will not function or will be modified, resulting in the generation of knock-out (Figure 5.8.) or genetically modified animals. The inserted DNA vector may include positive selection genes (e.g. an antibiotics resistance gene) or reporter genes (e.g. green fluorescent protein).

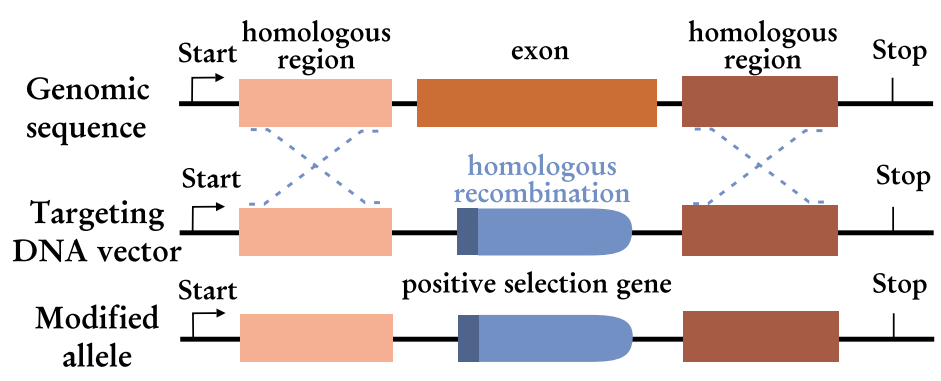

Figure 5.8. Knock-out allele created by homologous recombination: the positive selection gene introduced in the target gene replaces the original gene

The chance of homologous recombination is very low ( 1 in a million), therefore, the DNA vector has to be introduced into a very large number of cells in order to find transformed cells where the inserted DNA has been integrated into the target gene. In case if the DNA vector is introduced into embryonic stem cells (ES cells) by electroporation, cell colonies containing the targeted gene insertion can be obtained.

By injecting the stem cells into early-stage embryos (blastocyst) or aggregating them with 8-cell embryos, they will integrate among the cells of the host embryo and function as if they had always been part of the embryo. They get involved in the natural development of the embryo and contribute to the composition of all the tissues of the animal to be born. The animals generated this way are called chimeras, as a part of their tissues is formed from the cells of the host embryo, whereas other tissues are developed from the transgenic embryonic stem cells (Figure 5.9.). Gametes can also be generated from transgenic cells, enabling the establishment of transgenic mouse strains. In the frame of large scale international co-operations, several thousand transgenic embryonic stem cell lines have been developed, thus, as much as $10 \%$ of the identified mouse genes could be knocked out in these cell lines by now.

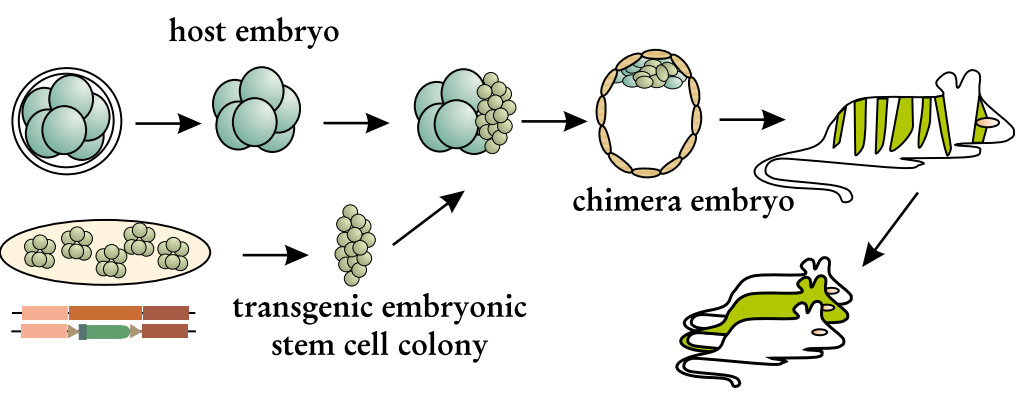

Figure 5.9. Transgenic stem cells injected into blastocyst stage mouse embryos are incorporated among the cells of the host embryo and participate in the development of the embryo, then chimera embryos and chimera progenies are generated. From the transgenic gametes of the chimera, transgenic progenies will be born

\section{Conditional knock-out animals}

Knocking out genes having important role in embryonic development might result in the early death of embryos, therefore the function of these genes could not be studied. Fortunately, researchers managed to develop a conditional knock-out procedure ensuring that the examined gene is removed only under certain conditions, for instance in a time- or tissue- specific manner, which can be achieved using the Cre-lox system. Cre is a bacterial recombinase enzyme which cuts the DNA between two loxP sequences. The loxP sequences can be inserted in the DNA vector, and as a result, knock-out will only occur in tissues where the Cre recombinase is also present. Conditional and targeted knock-out can be carried out by generating double transgenic mice through mating transgenic mice performing tissue-specific production of Cre recombinase enzyme with transgenic mice carrying the loxP sequence (Figure 5.10.). 


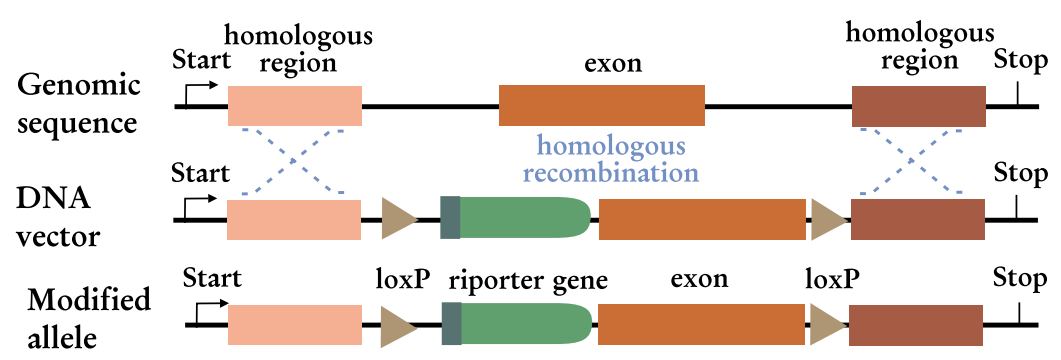

transgenic mouse carrying a

Cre recombinase gene

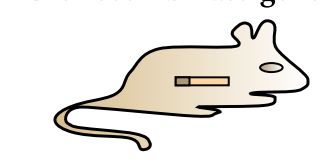

$\stackrel{\text { Start }}{\longrightarrow}$ Cre recombinase Stop

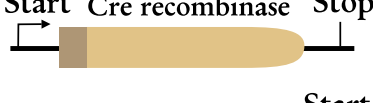

$\stackrel{\text { Start }}{\longrightarrow}$

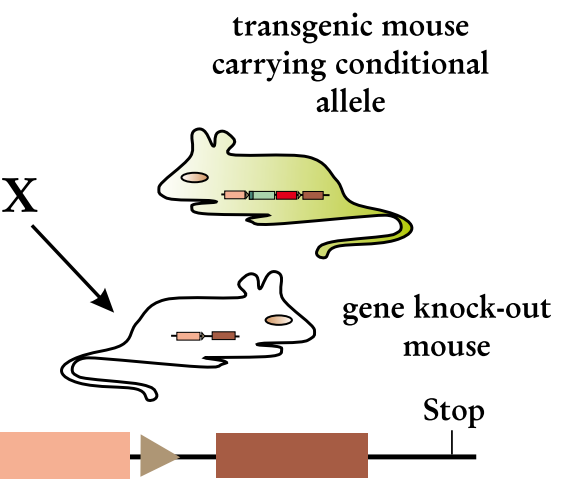

Figure 5.10. Conditional knock-out through the mating of GM mice producing Cre recombinase enzyme and GM mice carrying the loxP sequence. GM mice carrying the homologous recombination event can be identified using the reporter gene (at the top), then the completion of conditional knock-out is indicated in the progenies of the mated mice by the absence of reporter gene activity (at the bottom)

\section{Application of zinc finger nucleases to create knock-out animals}

The targeted knock-out of a certain gene can also be performed by the application of zinc finger nuclease (ZFN). This enzyme is involved in the repairing of defective DNA produced in the cells (incidental mutations) by identifying, as the first step of the procedure, the place of defect and cutting the DNA chain near it. In the second stage of the process, the repairing mechanism of the cell inserts the proper sequence in the DNA. ZFN molecules also ensure specific binding to the location of mutation and can now be designed to almost any DNA sequence; therefore, targeted knock-out can be obtained after cutting out any kind of DNA sequence. The application of ZFN enables knock-out also in animal species for which homologous recombination is not yet feasible (due to, for instance, the lack of stem cells). As a distant goal it was outlined that ZFN might be used for the specific repairing of genetic mutations as well.

\section{Transgenic animals generated by nuclear transfer}

Through the transferring of nuclei from somatic cells, not only the generation but also the cloning of transgenic farm animals carrying targeted genetic modifications has become feasible by now. DNA vectors can be introduced by electroporation (or using liposomes) into cultured cells derived from adult animals. By injecting the cells carrying the desired modification into the cytoplasms of enucleated oocytes, cloned embryos can be created which are then transferred to the uteri of pseudopregnant female animals to generate cloned progenies. Dolly, the first cloned transgenic lamb developed this way, produced a blood clotting factor that is highly important in human therapy. Once its efficiency is further improved, the method of cell nuclear transfer is expected to make various medical and agricultural applications feasible (Chapter 8).

\section{Conclusion}

It is important to realize that the vast majority of GM plants are generated in research laboratories and only a small proportion is intended for commercial product development. The purpose of these basic research experiments can be very diverse: discovery of a gene function, identification of a novel promoter, characterization of a metabolic pathway or gene interaction, understanding an environmentally regulated developmental process (e.g. the initiation of flowering, the functioning of the immune system), or the development of better transformation vectors and improved gene transfer techniques, etc. In the postgenomic era of possessing full genome sequences of the most diverse species, this knowledge is already of direct benefit for cutting-edge plant improvement and animal breeding programmes, e.g. by gene-assisted selection (GAS).

By a brief summary of the present status of gene transfer techniques and principles, this overview aimed at demonstrating that these are perfectly rational and reproducible methods routinely employed in a broad range of living organisms. Naturally (in the most appropriate sense of the word), there is always room for further improvement, and this will be really going on as long as science exists. However, the prompt practical application of the latest technologies is hindered in the case of GM organisms by extremely rigid regulation. The technology itself is mature enough, but the question is now more pointed than ever before: are we mature enough for its intelligent and 'selective' use? 


\section{Integration of gene technology research into plant breeding}

\author{
ZOLTÁN BEDÓ, CSABA L. MARTON
}

\section{Introduction}

Plant breeders can realize their objectives only if they develop genotypes suitable for knowledge-based agricultural production. This means, among others, that while up to now production and stability were increased primarily by boosting material input, in the future technologies potentially hazardous for the environment and human health would be replaced by plant varieties developed by molecular genetic breeding methods. These tasks present a new challenge for plant breeding, which can be met, in all probability, by integrated plant breeding utilizing the methods of both traditional and molecular breeding. The main objectives include:

- To preserve and, if possible, to enhance genetic diversity;

- To sustainably increase plant productivity with concomitant improvement of yield safety, including e.g. decreasing the pesticide load, breeding genotypes resistant to herbicides, fungal infection and insect infestation, improving yield stability by breeding cold, drought and salt tolerant genotypes;

- To reduce the area of land under agricultural cultivation, in order to protect natural environment;

- To produce food that promotes healthy eating, e.g. to boost vitamin content, to improve the nutrient transport of plants, to breed plants producing more of the essential amino acids, to increase the amount of bioactive components;

- To develop genetically modified plants that can be efficiently utilized for bioenergetic purposes;

- To improve the quality of life, e.g. by production of macromolecules utilizable for therapeutic purposes by the so-called "biopharming" procedure.

The aim of molecular plant breeding is to bring about changes on the DNA level in order to improve the agronomical performance or the biochemical parameters of a given plant, or to allow the development of a new, previously non-existent trait. In the course of the use of this technology, phenotypically testable properties are not hidden or affected by environmental factors, which is a constant problem for traditional breeders. Thus the efficiency of molecular breeding can be much higher than that of traditional selection carried out on the plant or population level; moreover, this method can even implement genetic modifications unaccomplishable by classical breeding methods. The toolbox of plant breeding has been enriched by powerful methods such as high-throughput genome analysis enabling researchers to screen plant gene banks and breeding materials for the determination of DNA polymorphism. The efficiency of genotyping is enhanced by the microarray technique allowing the systematic study of gene expression. It will become possible to identify and monitor genes participating in the individual processes. A deeper understanding of the plant genome will allow a more detailed insight into biochemical processes, the network of proteins and their metabolites and their interactions.

Plant gene technology is one of the new "tools" of molecular breeding, perhaps the best known to the general public. The Agricultural Research Institute of the Hungarian Academy of Sciences at Martonvásár has started plant breeding by genetic modification within the framework of an international cooperation, with the aim of engineering protection against a new, aggressive pest endangering Hungarian maize production. The results of this research program obtained to date are summarized in the case study below

\section{Breeding for resistance against the corn rootworm}

The first economic damage by the American corn rootworm was reported at the very beginning of the $20^{\text {th }}$ century in sweet corn. The corn rootworm is autochthonous in North America. It has three varieties, namely the Southern corn rootworm, the Western corn rootworm and the Northern corn rootworm, which differ not only in their choice of host plant but can also be differentiated morphologically. So far only the Western corn rootworm has shown up in Europe: its larval damage was first observed in a maize field close to the Belgrade Airport. Its spread in Europe has been continuous and quite rapid ever since. In Hungary the first imago was trapped in the summer of 1995. By now this pest is present in all the important maize-producing regions of Hungary.

Damage is mainly caused by the larvae, which feed primarily on the roots of the maize plant. Young larvae damage the root-hairs, whereas older larvae also attack thicker roots. Weakening of the root system leads to lodging and an 8-15\% yield decrease. The imago feeds on the leaves, stigma and pollen of maize; of these, stigma damage is the most important, because it causes fertilization problems in the ears.

In 1995 researchers already suspected that what they found was not only an individual of a new "domestic" species, but also the source of a new plant protection problem. In the course of more than a decade elapsed since the first finding, in the absence of natural predators corn rootworm has grown to be an agricultural pest. The speed of its spread is well demonstrated by the fact that local appearance of individuals of the species has already been recorded outside the Carpathian basin.

The corn rootworm has one generation per year; the eggs overwinter in the soil and hatch in the middle of May. The first imagos appear in the middle of June; females lay their eggs repeatedly, starting 10-14 days after hatching all the way to September. Their primary host plant is maize, but they are also capable of feeding on the roots of other plant species. They are sensitive to crop rotation and they can multiply the most successfully in monocultures. 
The elaboration of protective measures against the corn rootworm started in Hungary in 1996. The symptoms of larval damage on maize, the frequency of the pest's appearance and the average 5-10\% yield loss caused made the development of integrated pest control measures vitally urgent. Economically significant larval and imago damage was first observed in Hungary in 2000; the pest has been present continuously ever since, if in varying numbers.

\section{Pest control procedures}

The first suggested plant protection measures included crop rotation, insecticide dressing of seeds, soil disinfection, spraying of stands against the imago and sowing resistant hybrids. These efforts were later joined by biotechnology with its own specific approach, i.e. genetic modification of maize.

It has long been known that root lodging and the consequent plant death may cause extensive damage in plant monocultures. Plant stands damaged by corn rootworm are more sensitive to any biotic and abiotic stress than are nondamaged plant populations, especially under dry conditions.

When applying crop rotation, the growers make use of the observation that corn rootworm can only survive on the roots of maize and very few other crop plant species. Also, the eggs laying dormant in the soil gradually loose their vitality or even die in the second and third years. Therefore the basis of successful pest control is crop rotation.

In the course of crop rotation, some symptoms caused by newly invading imagos can be observed, but this rarely if ever reaches the economically detectable damage level. In the case of monocultures, however, the extent of damage may be as high as the entire yield, primarily due to the activity of larvae living in the soil.

In addition to crop rotation, another common pest control measure against corn rootworm is the application of effective insecticides. In Hungary as well as earlier in the USA, this type of pest control was practised on about $20 \%$ of the maize cropping area. The number of applicable agents has lately decreased, intensifying the risk of the development of insecticide-resistant biotypes. One such case has already been reported in the U.S.A.

It has been observed in Martonvásár that damage is particularly extensive in dry years. The extent of lodging and damage, however, is reduced by ample water supply before flowering. Plants are capable of regeneration within certain time limits, and to different extents depending on the developmental stage (phenophase).

According to our observations, regeneration depends on the frequency and amount of water supply (natural precipitation, irrigation), the size and resistance of the rootworm population and the diverse regenerativeabilities of maize varieties. Studies on regenerative ability may be a suitable tool in the hands of breeders using traditional breeding methods to improve corn rootworm tolerance.
The estimated costs of pest control amount to 4 billion HUF in Hungary and 40 billion HUF in the U.S.A. The cropping area of the USA is 30 -fold larger than the Hungarian area, whereas the costs of pest control are only 10-fold higher. This means that Hungarian growers spend three times more on chemical pest control per hectare than do American farmers. The reason for this may be that, on the one hand, damages are more devastating under dry conditions in Hungary, and, on the other hand, corn rootworm resistant hybrids are already widely used in the USA.

The interaction between the host plant and the insect pest may be interpreted by three basic mechanisms, namely preference, antibiosis and tolerance, of which conventional breeding can rely on tolerance. Differences in tolerance are mainly due to the diverse vegetative habitus of hybrids (stronger stem, larger and more massive root mass, more vigorous root regeneration). Transgenic plants exhibit true resistance, which is based on antibiosis.

\section{Breeding of transgenic maize}

Genetic modification is the introduction of a gene foreign to the plant species in question and its operation there. "Bt" is the abbreviation of the name of the spore-forming soil bacterium Bacillus thüringiensis. The spores and the endotoxin of this bacterium have been used in plant protection technologies as a pesticide of microbial origin (Dipel) since the 1950s. The gene introduced into maize produces a crystalline protein (hence the designation of the gene, i.e. crystal (cry) with insecticidal properties, which in itself is not toxic (a so-called protoxin). It becomes toxic when its structure is changed inside the digestive tract of the larva or imago feeding on the plant, an event dependent on signal transduction processes.

The protoxin produced in the cells of the plant is consumed by the larva and gets into its digestive tract, where it binds to receptors of the midgut and causes the death of the larva by damaging the membrane of the epithelial cells. The crystalline protein is not soluble in neutral or acidic medium. The alkaline $\mathrm{pH}$ of the larval gut, however, promotes dissolution, and the protoxin is then activated by digestive enzymes in the gut. The active protein moves through the membrane of the larval gut to the epithelium of the midgut. In the target insects the activated protein recognizes special receptors on the surface of the gut cells and binds to them. Its binding paralyses gut function, as a result of which the larva stops feeding and dies.

The toxins produced by the various bacterial strains have different target species. At present maize hybrids producing Cry9F, Cry1F and Cry1Ac toxins effective against certain Lepidoptera pests (e.g. European corn borer) and Cry3A, Cry3Bb1, Cry34Ab1 and 35Ab1 toxins targeting Coleoptera pests (e.g. corn rootworm) are grown globally.

Thus, protoxins produced in the plant are species specific, i.e. they do not harm all living organisms, only a given number of target species. Consequently, their activity spectrum is significantly narrower than e.g. that of an insecticide used 
for soil disinfection. Like all Cry proteins produced by Bacillus thüringiensis, the protein effective against the corn rootworm also has to be consumed by the insect in order for the insecticidal effect to manifest (Figure 6.1.).

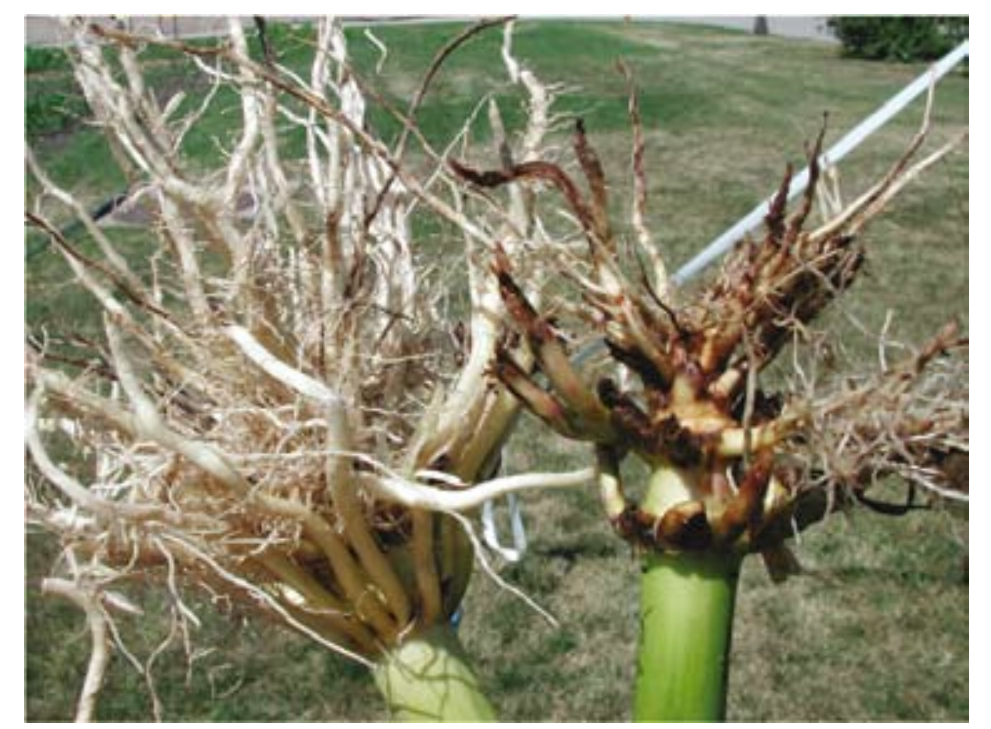

Figure 6.1. Transgenic (left) and traditional (right) maize roots infested by corn rootworm

Another important question is which plant parts contain the active agent. The larva of the corn rootworm feeds on the roots, whereas the imago damages practically all other plant parts: it feeds on the stigma and the pollen, it peels the leaves and even gnaws at the kernels. The Bt genes are equipped with a regulatory region (a so-called constitutive promoter region) that makes them expressed in all parts of the plant, thus all cells, tissues and organs of the plant produce the protoxin protein. The level of expression is different in each organ: the protein product is present at the highest concentration in the leaves, whereas its amount is negligible in the pollen. The order of plant organs according to the amount of toxin produced is the following

leaf $>$ anther $>$ root $>$ stem $>$ seed $>$ pollen

The appearance (phenotype), "behaviour" and developmental cycle of Bt plants do not differ at all from those of the corresponding non-transgenic plants. The composition of $\mathrm{Bt}$ maize was compared to that of traditional maize varieties grown in the same experiments in the USA. 77 different parameters were analysed including moisture content, protein content, amino acids, glycosides, fats, fibres, vitamins, various secondary metabolites, minerals and ash. Neither of these parameters was found to show a biologically significant difference. With regard to composition, Bt maize can be considered equivalent with traditional maize (except for the added characteristics). Bt maize, however, is definitely resistant to insects: the biotechnological method is capable of killing nearly $100 \%$ of insect larvae.

Some maize hybrids also carry another transgene linked to the Bt gene, a gene conferring resistance to the herbicide Roundup. Its effect is quite spectacular: on fields sprayed with the adequate total herbicide, weeds and non-transgenic maize plants are killed off. The resistance of the Roundup Ready maize varieties is stable and is expressed at high levels, thus the plants stay alive and their growth and development are not arrested.

Herbicide resistance is due to the modification of a single gene that is originally present in the plant. Due to the expression of the glyphosate-tolerant 5-enolpyruvyl-shikimate-3-phosphate synthase (EPSPS) derived from an Agrobacterium strain, RR maize exhibits resistance to the wide spectrum agricultural herbicide Roundup, whose active agent is glyphosate.

At present, field production of the above mentioned transgenic hybrid maize varieties is not authorized in Hungary: production of the GM varieties otherwise approved in the European Union (MON810) and included in the national catalogue is under a unanimously adopted moratorium. Applications for the approval of the field release of corn rootworm resistant and/or herbicide tolerant maize hybrids are at present under scrutiny in the European Union.

\section{Summary}

The American corn rootworm (Diabrotica virgifera virgifera LeConte) has become one of the most important pests of maize in Hungary. It is present in all significant production areas, and in several regions, where the loss caused exceeds the economic damage threshold, it has become the most problematic of biotic and abiotic stress factors.

At present the only options of pest control are the use of insecticides (soil disinfection, seed dressing, spraying) and crop rotation. There exist some maize hybrids with more than average tolerance, but this trait in itself does not provide protection. The solution of the future could be the use of transgenic hybrids that have true resistance to the corn rootworm, protecting the plants not only in the first stage of the vegetative period, like seed dressing and soil disinfection, but also throughout the entire life cycle. Research has already been started at Martonvásár within the framework of international cooperation. Production of corn rootworm resistant maize hybrids is already approved in the USA, and this is the most important method for pest control. 


\section{Present and future role of different generations of GM crops in the Hungarian agriculture}

\section{DÉNES DUDITS}

"For the prospective effects of a responsible use of GM crops, one could expect that within the 21st century specific knowledge from genomics, proteomics, systems biology and metabolomics can form a rich basis for a realistic progression of biotechnological contributions towards an increasing sustainability of agriculture."

Werner Arber, Nobel Laureate, (Biotechnology Advances, 2009, 27: 940).

\section{Introduction}

The quantity and quality of yield from crop plants are determined by the combined effects of various factors functioning in plant production systems. Fruits of the farmer's work is ensured in a complex manner by the interaction of biological, climatic, soil, technological and economic parameters. The plant's productivity is limited biologically by the genome of the seed sown. This is why plant breeding has a leading part when the yield potential is considered as a basic component of sustainable development, since, in order to maximize yield, the metabolic processes of plants has to be improved continuously through the optimization of gene composition. The history of several centuries of plant breeding confirms that the yield of varieties can be increased by crossing, selection, the multiplication of chromosome number and the induction of mutations. The success of breeding has always depended greatly on the application of the findings of biological and genetic research, as proved also by the results of Hungarian plant breeding, whether we consider the development of wheat, sugar-beet, hybrid maize, alfalfa, fruit, grape or floral plant varieties. This tradition would be interrupted if today's plant breeders disregarded the statement of Arber Werner quoted above and did not employ gene technology methods for the 'manipulation', the improvement of plant genomes. In Hungary, the multi-frontal attack against GM plants seems to have been very successful so far, as demonstrated by the moratorium and the biased legislation ensuring prohibition. Today, the ideological and political debates turn into abuses of power when administrative means are used to obstruct even the research of GM plants, and researchers are intimidated, their manuscripts are censored. This policy interfering with our long-term national and economic interests is able to succeed in spite of the fact that it is inconsistent with the agricultural strategy of the European Union and the developmental trends in global agriculture. A clear message is provided in the declaration by Máire Geoghegan-Quinn, the European Commissioner for Research, Innovation and Science: "Crop production will have to cope with rapidly increasing demand while ensuring environmental sustainability. Preservation of natural resources and the need to support the livelihoods of farmers and rural populations around the world are major concerns. In order to achieve the best solutions, we must consider all the alternatives for addressing these challenges using independent and scientifically sound methods. These alternatives include genetically modified organisms (GMO) and their potential use." Basically, this assessment is affirmed by the worldwide tendencies in the production of genetically modified (GM) plants.

The marginal importance of GM plants authorized to date in the European Union in the variety usage in Hungary: ideological and political overreaction

According to the most recent statistics, GM plant varieties were cultivated in 2010 in 29 countries worldwide on 148 million hectares. Out of the 15,4 million growers, 14,4 million belonged to the group of resource poor small-scale farmers. This information unequivocally denies the frequent criticism that genetically engineered varieties only serve the interests of large-scale producers. Part of the currently cultivated GM plants is indifferent for Hungarian agriculture. Although they may provide considerable economic and environmental benefits in certain countries, insect-resistant cotton or virus-resistant papaya is not intended to be grown in Hungary. GM soybean is the most widespread transgenic plant and herbicide tolerance is the most often modified feature; still, varieties with these resistances are not in primary demand at present, due to the unexploited potentials in Hungarian soybean production. While there are 300 thousand hectares in the country suitable for the production of this crop, soybean was only grown on 20-50 thousand hectares in recent years. Increasing the soybean cropping area is reasonable, and the decision on the varieties to be grown may be influenced by the lower production costs of GM plants; on the other hand, the yield of traditional varieties can be marketed at a premium. The case of Romania is an instructive example for the consideration of technological issues of Hungarian soybean production. In Romania, herbicide resistant GM soybean had been produced since 1999, and was grown on 130 thousand hectares in 2006 . As of the date of the country's EU accession, 1 January 2007, the cultivation of GM plants had to be given up, resulting in a dramatic decline in soybean production, since farmers are not interested in growing much more expensive and less efficient 'traditional' soybean varieties. According to the Romanian Minister of Agriculture, „Romania looses one thousand million euros each year because of the prohibition of GM soybean production, as this forage has to be imported from Brazil" (Agerpres News Agency).

The choice of GM maize hybrids in Europe is very limited, since only the hybrid resistant to the infestation of European corn borer, MON810 is authorized for production. The yield loss attributable to corn borer is $3-5 \%$ nationwide; however, more severe infestation may be expected in certain areas and fields. The pest damages almost all the important parts of the plant, and besides 
causing direct damages, it facilitates secondary infections, among others Fusarium. With regard to the extent of expected economic loss, corn borer resistant hybrids producing the protein Cry $1 \mathrm{AbC1}$ are not likely to reach a considerable share in Hungary's maize production, even if farmers are assumed to freely choose among varieties. Still, a very intensive anti-GM campaign has been incited in the country, gaining political and media support for a moratorium. Among the member states of the European Union, Hungary's government officers are committed opponents of gene technology and advocate the suppression of this technology in all community forums. Simultaneously, the public is successfully misinformed. Farmers are also provided with many kinds of misleading information and they do not even have the chance to try these varieties in a small scale. As shown in Figure 7.1., Hungarian cereal producers get acquainted with the results of the production of GM plants in Romania. Also, Slovakian GM maize fields could only be seen by few Hungarian maize producers

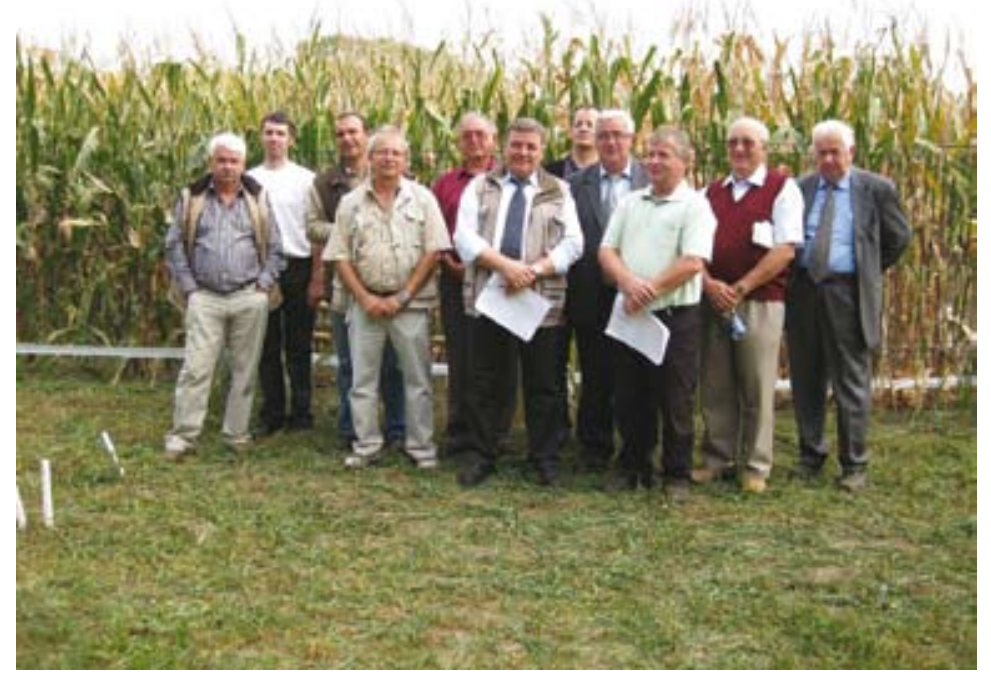

Figure 7.1. Hungarian farmers in Lovrin (Romania) where they study GM corn fields (István Fehér, 2010, Zöld Biotechnológia, 1: 4)

The second GM plant approved after a decade-long authorization procedure is the potato 'Amflora' in which the starch structure was modified by gene introduction in order to increase the economic profitability of industrial processing. The news about its authorization in the EU triggered a shocking effect: the Hungarian media frightened the public with the antibiotic resistance gene, and the responsible ministry initiated legal proceedings, despite the fact that this variety is indifferent to Hungarian potato growers. The case of the GM potato made it clear that scientific facts have no weight in an acrimonious ideological dispute. With regard to the Hungarian moratorium, official arguments refer to a considerable economic benefit. However, no actual facts are known, though accurate assessment of the situation would be greatly needed, as the present anti-GM approach generates substantial damages in agricultural innovation and delays the preparation for the production of newer generations of GM plants and the application of related technologies.

World tendencies of plant breeding using gene technology and newer generations of GM plants: domestic examples of development

While in Hungary all the opponents' attacks are concentrated on the two GM plants authorized in the EU, genome programmes are in progress and the complete genetic information of an increasing number of field crops becomes known, considerably expanding the possibilities of breeding by genetic engineering. The vast amount of new information and the progress in getting acquainted with the wonderful life of plants are very impressive. All these result open a completely new dimension to the role of biotechnology in the management of global problems and sustainable development. A great proportion of published scientific articles apply the method of gene transfer. It is hard to find a plant trait or function that is not studied by using GM plants. In such a rapidly developing scientific environment, there is nothing special in that the many kinds of GM plants generated are assessed for their agronomic properties as well. Gene technology solutions that constitute novelties and have potential economic value are patented in accordance with the demands of the innovation systems. Although the authorization activity of the EU is in great delay, further generations of GM plants are developed one after the other. The success of Chinese research and the diversity of the GM plants under authorization are worth taking into account, as the GM-based plant production in China might have a global effect. When considering Hungarian agricultural strategy, it might be important to bear professional predictions in mind and to give up the political fallacy that researchers asserting the importance of gene technology are the paid agents of multinational companies: this is a ridiculous and harmful argument.

Figure 7.2. presents the analysis by Stein and Rodriguez-Cerezo of the current numbers and the estimates of future numbers of transgenic events per species. It can be seen that besides cotton and rice, it is the group of 'other' plant species where the number of new gene transfer events are expected to increase significantly. The data in Table 7.1. forecast that the role of insect and herbicide resistant plants will remain dominant up to 2015 . The number of genotypes with improved quality traits will only increase slowly. Based on the known research and development trends, the appearance of GM plants representing value for Hungarian plant producers may be taken for granted. First of all, hybrids resistant to corn rootworm can be mentioned, since chemical control measures against this pest cost 4 thousand million Hungarian forints every year. The role of drought tolerant varieties and those with increased capacity for nitrate and phosphorus utilization is undeniable. The increased recognition of the importance of GM wheat is also worth examining. 


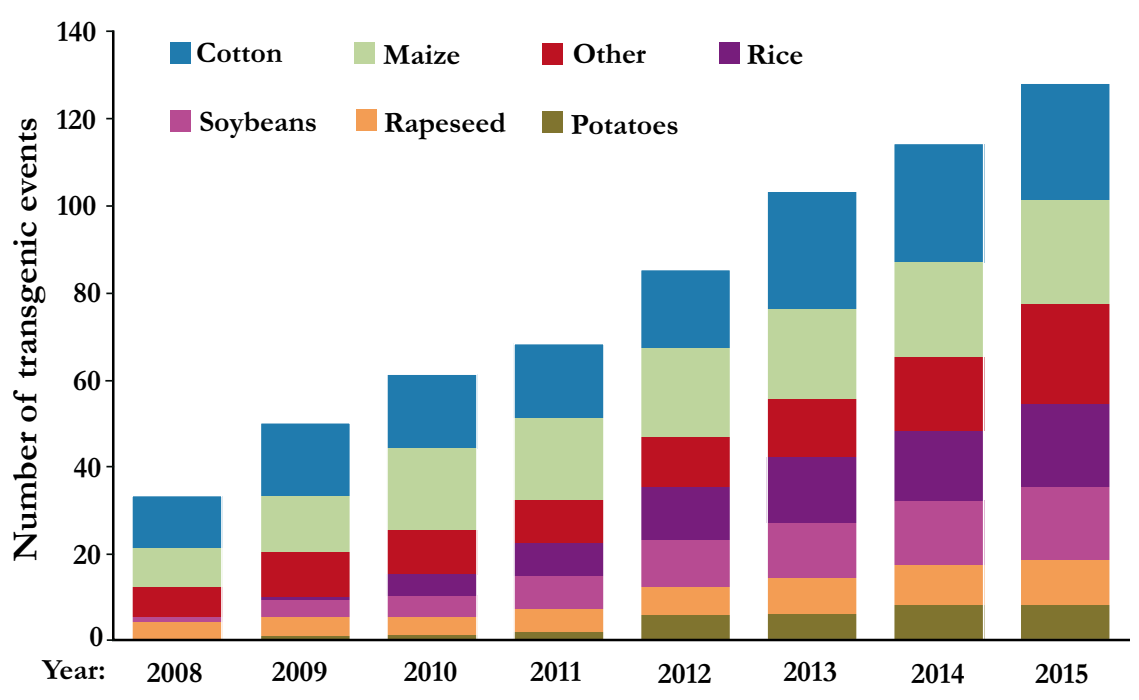

Figure 7.2. Current numbers and estimations of future numbers of GM crops worldwide (Stein and Rodroguez-Cerezo, 2010, Nature Biotechnology 28 : 23)

\begin{tabular}{|c|c|c|c|c|c|}
\hline $\begin{array}{l}\text { Trait } \\
\text { category }\end{array}$ & $\begin{array}{l}\text { Commercial } \\
\text { in } 2008\end{array}$ & $\begin{array}{l}\text { Commercial } \\
\text { pipeline }\end{array}$ & $\begin{array}{l}\text { Regulatory } \\
\text { pipeline }\end{array}$ & $\begin{array}{c}\text { Advance } \\
\text { development }\end{array}$ & $\begin{array}{c}\text { Total by } \\
2015\end{array}$ \\
\hline $\begin{array}{l}\text { Insect } \\
\text { resistance }\end{array}$ & 21 & 2 & 11 & 25 & 59 \\
\hline $\begin{array}{l}\text { Herbicide } \\
\text { tolerance }\end{array}$ & 11 & 5 & 4 & 13 & 33 \\
\hline $\begin{array}{l}\text { Product } \\
\text { quality }\end{array}$ & 2 & 1 & 5 & 12 & 20 \\
\hline $\begin{array}{l}\text { Virus } \\
\text { resistance }\end{array}$ & 5 & $\mathbf{0}$ & 2 & 3 & 10 \\
\hline $\begin{array}{l}\text { Abiotic stress } \\
\text { tolerance }\end{array}$ & 0 & 0 & 1 & 6 & 7 \\
\hline Other & 0 & 0 & 2 & 11 & 13 \\
\hline
\end{tabular}

Table 7.1. Numbers of current and possible numbers of expected GM traits worldwide (Stein and Rodroguez-Cerezo, 2010, Nature Biotechnology 28: 23)
Competitiveness is denoted by the extent of expenditures on green biotechnological development. As was recently reported by Maive Rute, EUR 200 million has been invested in GMO research and development through the Framework Programmes since the year 2001. Considering the objectives, it can be established that priority was given to projects related to detection, risk assessment and co-existence. The development of original GM technologies has been given little financial support. At the same time, the company Monsanto alone spent 1 thousand million dollars on such improvements in 2010. China started the 'transgenic green revolution' in 2008 with a budget of 3.5 thousand million dollars. Under such circumstances, the competitive disadvantage of the European Union is no mere chance.

Despite the fact that the Hungarian administration has adopted a negative attitude towards the production of genetically engineered plants, basic research is intensively pursued in the country, as demonstrated by the presented list of publications (Chapter 19). Several cultivated crops have been involved in the development and adaptation of gene introduction techniques. Among early initiatives, the development of alfalfa carrying the kanamycin resistance gene can be mentioned, which was an internationally recognized novel result in 1986. Development of gene transfer methods has often been conducted in collaboration between molecular biologists and plant breeders. Successful breeding of a maize genotype capable of embryogenesis and plant regeneration from somatic cells (Sándor Mórocz) enabled the development of herbicide resistant maize in cooperation with the company Hoechst (Figure 7.3.)

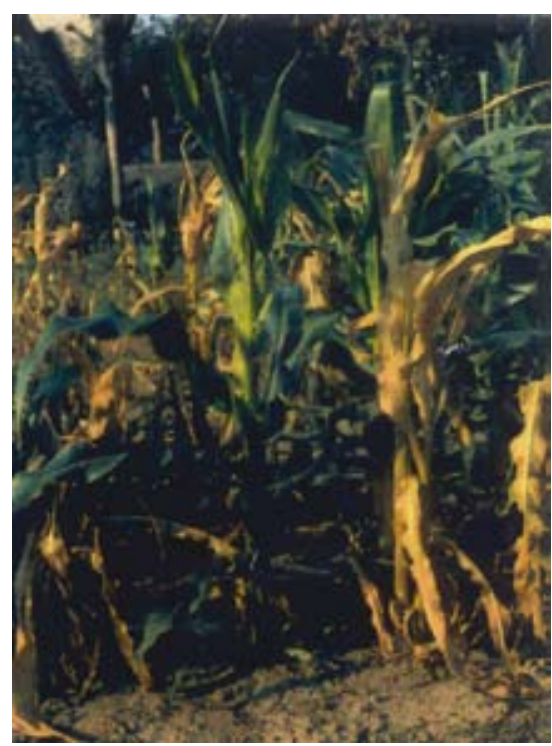

Figure 7.3. Mendelian segregation for phosphinotricin resistant (left) and sensitive (right) plants as progenies of transgenic maize plants, developed from protoplasts (Omirulleh et al., 1993, Plant Molecular Biology, 21: 415) 
This technology was marketed internationally as well. The symptom-free state of the virus resistant tobacco lines generated by Ervin Balázs and his team is illustrated in Figure 7.4.

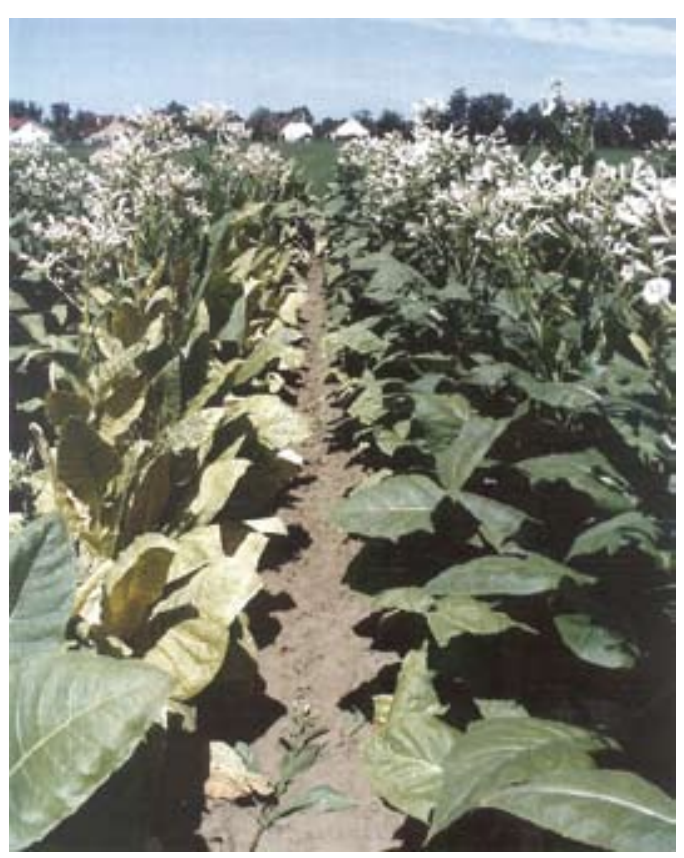

Figure 7.4. Expression of Y-virus coat protein genes provides virus resistance of tobacco plants (right) while the control plants are severely damaged by the virus infection (Balázs et al., Agricultural Biotechnology Center, Gödöllő, Hungary)

A key subject of Hungarian gene technology research is the improvement of the stress tolerance and pathogen resistance of plants. The research team of Zsófia Bánfalvi is involved in innovative gene transfer experiments with the purpose of increasing the stress tolerance of potato (Figure 7.5.). Plants resistant to bacteria and fungi have been created in cooperation with research institutes of the Hungarian Academy of Sciences as well as in the Agricultural Biotechnology Center.
WL
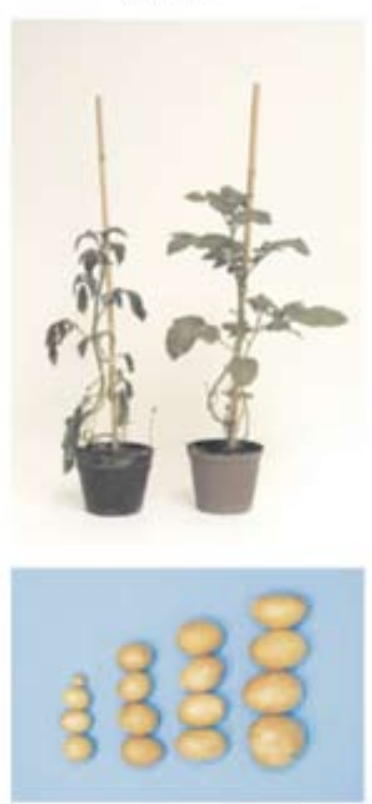

\section{WL-TPS}
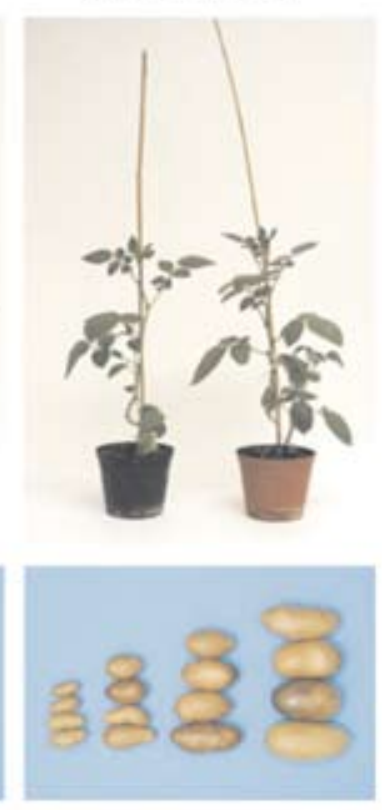

Figure 7.5. Improved drought tolerance of potato plants after expression of trehalose-phosphate-synthase gene under the control of drought inducible promoter. (WL) wild type, (WL-TPS) transgenic plants: reduced watering (left), well watered (right) (Stiller et al., 2008, Planta, 227: 299)

The success of Hungarian wheat breeders is well demonstrated by the fact that $70-80 \%$ of domestic wheat fields are sown with Hungarian varieties. Breeders at both Martonvásár and Szeged wish to be prepared for the development of the new generation varieties; therefore, intense research has been conducted in the field of molecular resistance breeding. The GM wheat plants shown in Figure 7.6. have been developed in Szeged and, due to the over-production of a detoxification enzyme, suffer less damage under conditions of water deficiency. The examples cited above convincingly demonstrate the success of gene technology research conducted in Hungary. In order to enable the use of breeding material generated in this way in current breeding programmes, administrative obstacles hindering the expansion of field trials would have to be eliminated. The plant breeding and gene technology traditions in Hungary provide a firm basis to support our researchers and breeders, as active participants, in the introduction of the new generations of plant production technologies. In consideration of the new agricultural technologies arising out of worldwide 
research and development processes, it is unimaginable that the innovation disadvantage of Hungarian farmers be maintained for a long period of time. Also, to ensure the competitiveness of domestic small- and large-scale farms, a free choice of varieties is essential, particularly if the production of GM varieties will not be prohibited in the neighbouring countries.

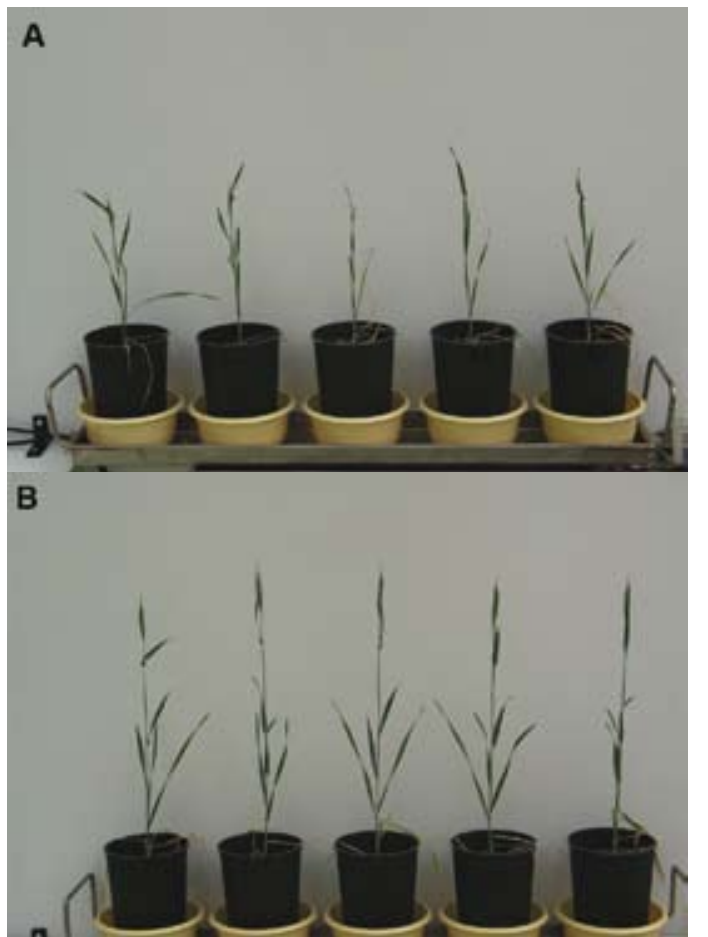

Figure 7.6. Expression of alfalfa detoxification gene caused drought tolerance in wheat plants. (A) control plants, (B) transgenic plants. (Pauk et al., Cereal Research Non-Profit Company, Szeged; Biological Research Centre, HAS, Szeged)

\section{The results and future prospects of genetically modified livestock animals}

\section{ZSUZSANNA BÓSZE, LÁSZLÓ HIRIPI}

At its 2009 World Food Summit the United Nations Food and Agricultural Organisation recognised that agricultural output will need to increase by $70 \%$ till 2050 in order to feed the world's population, which is expected to exceed 9 billion in this timeframe (FAO 2009, ftp.fao.org/docrep/fao/Meeting/ 018/k6050e. pdf). It will be necessary to achieve this rapid increase in production on the same amount of, or less agricultural land. The global demand for animal products is also substantially growing, driven by a combination of population growth, urbanization and rising incomes. There is little likelihood that vegan diets will be acceptable or prevalent in the medium term, therefore it would be unwise to build strategies for achieving food security upon assumptions of altruistic or government advised changes in eating behaviour. In the past half century major productivity gains in animal husbandry were realized along with reductions in the greenhouse gas emissions and global warming potential per ton of animal product. The dramatic improvements can be attributed in large part to the algorithms developed by quantitative geneticists to predict the potential genetic merit of offspring (Hume et al., Journal of Agricultural Sciences, 2010, in press).

Recent whole genome sequencing programs in livestock animals revolutionized genetic selection based on whole genome genotyping, genomic selection (GS). Although predicting genetic merit using DNA diagnostics may be less precise than directly testing the performance of every animal, the reduction in generation interval by far offsets it. However, even this approach has limitations, since it relies on existing genetic variation. If a trait such as disease resistance does not exist in the population, it is not possible to select for it.

Genes are not equal in their effects upon changes in phenotype. In general terms genetic modification is appropriate to add major effect genes, whereas genetic selection is applied to all genes including the lesser-effect genes. Figure 8.1. describes the effect of genetic selection and transgenesis on genome modification. Those facts, on the one hand, underline the importance of research projects like NEXTGEN (European $7^{\text {th }}$ Framework program), for preserving the existing biodiversity in livestock species, and on the other hand they point out the importance of transgenic technologies, which have the unique potential to introduce desirable traits through crossing species barriers. The pioneering PEGASUS project (European $7^{\text {th }}$ Framework program) aimed at exploring public perception of genetically modified animals in science, utility and societal perspectives (http://www.projectpegasus.eu). 


\section{Standard selection}

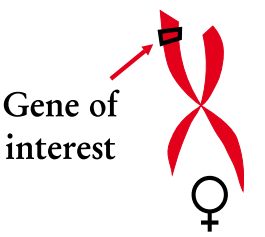

Chromosomes $\Upsilon^{7}$

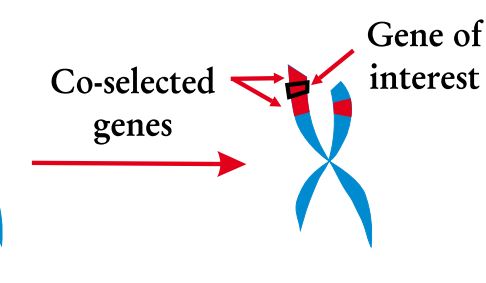

Transgenesis

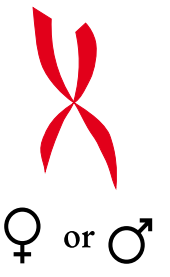

Gene of interest

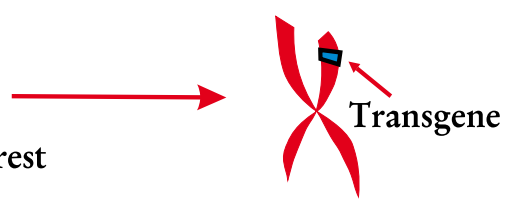

Figure 8.1. Impact of genomic selection and transgenesis on genome modification (adapted from Houdebine and Jolivet, 2011, Trends in Food Science \& Technolog, in press)

Classical genetic selection relies on the recombination of homologous chromosomes during gamete formation and the random distribution of parental genes to progeny. Transgenesis provides organisms in one generation with exogenous genes that have known and potentially useful properties.

Initially transgenic technology was developed as a research tool to study gene function in mice, but a group of scientists proposed that this technology might have immediate applications for both agricultural and biomedical purposes, when extended to livestock animals. Their vision started to become reality in the last few of years. However, it should be emphasized that large animal research is expensive. Over the past 20 years there has been systematic underinvestment by governments, especially in the EU. This is not a sector that can be left solely to industry investments.

The biomedical applications of transgenic livestock animals are in a more advanced stage due to greater economical incentive and public acceptance. The main biomedical applications of GM livestock are (1) biopharming (live bioreactors) for large-scale production of pharmaceutically important proteins, (2) xenotransplantation: organ donors for animal to human transplantation and (3) livestock animal models for human diseases.
The main areas of the agricultural applications of transgenic technology are (1) increased production efficiency, (2) improved animal welfare and health, (3) improved food safety and quality and (4) reduced environmental footprint.

\section{Biomedical applications}

Recombinant protein expression in the milk of GM animals has been extensively studied in the last twenty years and has recently undergone improvement both from a methodological point of view and in terms of reaching the market. The U.S. FDA issued its first approval for a biological product produced by genetically engineered animals in 2009, for ATryn (human antithrombin III) to treat a rare clotting disorder (www.fda.gov/NewsEvents/Newsroom/PressAnnouncements/2009/ucm109074.htm). Patients with hereditary antithrombin deficiency are at high risk of blood clots during medical interventions, such as surgery, and during and after childbirth. ATryn is a therapeutic protein derived from the milk of goats, and the manufacturer is GTC Biotherapeutics, Inc. (www.gtc-bio.com). The amount of ATyrn obtained per year from transgenic goats is equivalent that obtained from 90000 human blood samples. Other pharmaceutical proteins produced in bovine, goat or rabbit milk are in the pipeline, at different phases of clinical trials. The European Union harbours quite a few biotechnology companies, whose proprietary technology is based on genetic modification of animals. Notably, the Dutch biotech company Pharming Group received the Award for Best Biotech Company at the World Technology Summit in 2010. Pharming has been awarded for "its innovative work on production of life enhancing drugs derived from its transgenic platform." They received European approval for their lead product Ruconest for treatment of hereditary angioedema (www.pharming.com). Ruconest is produced in the milk of transgenic rabbits, and it is the second transgenic animal-derived drug on the market.

ImmunoGenes AG (www.immunogenes.com), a spin-off company of the Eötvös Lóránd University and the Agricultural Biotechnology Center, Hungary was founded in 2007 to develop a technology whose underlying mechanism is overexpression of a special receptor that is crucially involved in enhancing the humoral immune response. The genetically modified rabbits and mice have superior antibody producing system, therefore are more suitable for increased, high quality polyclonal and monoclonal antibody production, saving costs, time and animals. ImmunoGenes was Semi Final Winner at the Eurocan European Venture Contest 2009. The Laboratory of Chromosome Structure and Function of BRC in Szeged. Hungary has developed a methodology for the in vivo generation of mammalian satellite DNA based artificial chromosomes with defined genetic content, suitable for safe gene therapy and for production of therapeutic molecules in transgenic animals (Duncan and Hadlaczky, 2007, Current Opinion in Biotechnology, 18: 420)

Other viable alternatives as sources of recombinant proteins are birds' eggs. Poultry transgenesis has become efficient using 3 rd generation lentiviral systems 
with enhanced biosafety features. Recombinant protein can be cost efficiently produced in egg white with high purity. The cost of the purified recombinant protein is calculated to be $1-10 \%$ of that of mammalian origin. Production of antibodies and human interferon in egg white is at the research and development stage. Exotic domestic animals like domesticated silk moth are also going to enter the biopharming business. Transgenic silk moth larvae produce recombinant proteins in the cocoon made up of raw silk. Japanese researchers consider silk moth as a living bioreactor, a competitive alternative method.

Unfortunately even this approach, namely the production of recombinant proteins by livestock animals is developing more slowly than it potentially could. Pharmaceutical companies may be reluctant to adopt these techniques just because they are new, and because they suffer from the negative image of GMOs, but perhaps also because they presently make substantial profits with proteins prepared from cultured cells.

\section{Xenotransplantation}

The shortage of donated human organs for transplantation continues to be a life threatening problem for patients suffering from complete organ failure, and this gap is increasing in Western populations. Alternatives to the use of human organs for transplantations include stem cell therapy and organs from other species, i.e. xenografts. Pigs are currently thought to be the best candidates for organ donation. The risk of cross-species disease transmission is decreased because of their phylogenetic distance from humans. They are readily available, their organs are anatomically comparable in size, and new infectious agents are less likely since they have been in contact with humans through domestication for many generations. Current experiments in xenotransplantation most often use GM pigs as donors and baboons as human models. The barrier to pig-to-primate xenotransplantation most difficult to overcome is rejection of the grafted organ by a cascade of immune mechanisms commonly referred to as hyperacute rejection, acute humoral xenograft rejection, immune cell-mediated rejection, and chronic rejection. In Europe not more than a handful of experts work on xenotransplantation projects, among them H. Niemann and co-workers at the Institute of Farm Genetics in Mariensee, Germany. The protective efficacy of all strategies is strictly dependent on a sufficiently high expression level of the respective factors with the required spatial distribution. A future challenge will be to combine the most important genetic modifications in multi-transgenic pigs for clinical xenotransplantation. The development of these pigs is in experimental stage, but is expected to reach clinical trials in five years.

\section{Livestock animal models for human diseases}

The domesticated pig has emerged as an important disease model, and the object of preclinical testing of novel therapies in human brain disorders. The volume of available background data concerning pig brain anatomy and neurochemistry has increased in recent years. The pig's brain resembles the human brain more in anatomy and development than do the brains of small laboratory animals. The size of the pig brain permits the easy identification of cortical and subcortical structures. A Danish research group at the University of Aarhus created the first porcine model of Alzheimer's disease in transgenic minipigs (Kragh et al., 2009, Transgenic Research, 18: 545.).

Just like pigs, rabbits as experimental animals also represent a more appropriate model than rodents in different biomedical aspects. Especially early prenatal development, its long-lasting effects on health and complex diseases (diabetes mellitus, hypertension, atherosclerosis, hyperlipidemia and obesity) cannot be adequately mimicked in mice. GM rabbits expressing the green fluorescent gene provide a way for the visualisation of tissue anatomical structure and cell morphology through in vivo imaging (Figure 8.2.). In addition, transgenic rabbit models of human disorders connected to cardiac electrophysiology and cardiac hypertrophy have turned out to be extremely useful in disease prevention and pharmacogenomic studies (Senthil et al., 2005, Circulation Research, 97: 285; Bentzen et al., 2010, Journal of Cardiovascular Pharmacology, Nov. 26. Epub ahead of print).

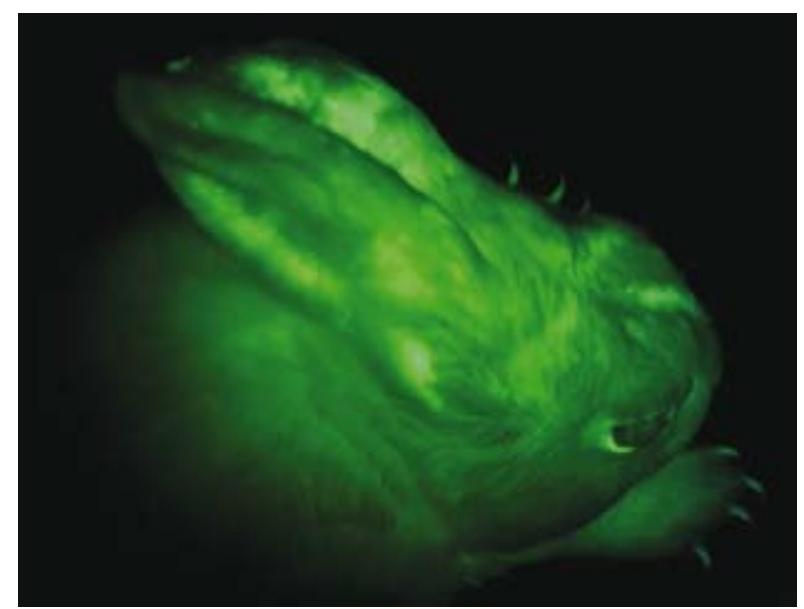

Figure 8.2. Transgenic rabbits expressing the green fluorescent protein (Hiripi et al., 2010, Transgenic Research, 19: 799)

\section{Agricultural applications}

\section{Increased production efficiency}

Given the genetic diversity that is available for selection and propagation, there are theoretical limitations to the production capacity of livestock. It is unlikely that existing genetic variation will continue to generate the rate of gain obtained 
in the past. It is very likely that genetically modified animals will be required and that they will be accepted. After nearly 15 years of research and development the transgenic Atlantic salmon expressing the chinook salmon growth hormone gene are already on the way to the table, albeit with considerable opposition from environmental groups. Environmental and bioethical problems are being considered and this project is close to an agreement by ad hoc committees (www.fda.gov/NewsEvents/PublicHealthFocus/ucm224089.htm)

\section{Improved animal welfare and health}

Transgenic cattle produced in the USA express the antibacterial protein lysostaphin in their milk, which dramatically enhanced the resistance of these cows to infection by Staphylococcus aureus, the most common cause of mastitis. This genetic improvement could improve the well-being of millions of dairy cattle and decrease the economical costs of mastitis (\$2 billion per year in the USA). Among the limitations of the agricultural approaches are (i) the slowness of the dissemination of the transgene in herds through artificial insemination as compared to plants, and (ii) the lack of public acceptance, which may shy away potential producers.

Beyond that, any GMO strategy would need to compete on a cost-benefit basis against vaccination and other disease prevention strategies to become the preferred disease prevention policy. Currently, there are no treatments for more than half of all diseases that affect animals. Even in the case of diseases for which treatment is available, some issues limit their effectiveness, e.g. virus variants against which the vaccine is not protective, or the global concerns about the extensive use of antibiotics (Wall et al., 2009, CAST Issue paper 43, Animal Agriculture's Future through Biotechnology, Part 8). Transgenesis offers novel disease prevention strategies, like RNA interference. This strategy is very promising to combat virus-caused diseases (foot and mouth disease, porcine reproductive and respiratory syndrome, avian influenza - Long et al., 2010, Reproduction, Fertility and Development, 22: 47). In January 2011 it was a breakthrough in animal biotechnology, when scientists from the Roslin Institute and the University of Cambridge reported the successful generation of transgenic chickens made with a lentivirus expressing a small RNA that interferes with the RNA polymerase from avian influenza virus, and, hence, with its replication. Transgenic chickens infected with avian flu virus $\mathrm{H} 5 \mathrm{~N} 1$ became sick but failed to transmit the infection to other chickens kept in the same pen with them (Lyall et al., 2011, Science, 333: 221). It is generally agreed that further studies are needed to ensure that GM chickens do not suffer from side effects and are safe for consumption.

\section{Improved food safety and quality}

Current production systems provide safe animal food products with good nutritional qualities, but there is room for improvement. Functional foods are increasingly fashionable in the industrialized world. Milk is an important foodstuff and therefore introduction of antimicrobial properties into milk could be beneficial for the consumer. GM goats that are producing the human antibacterial protein lysozyme in their milk were created in the USA (Maga et al., 2006, Journal of Dairy Science, 89: 518). Their milk was consumed by pigs as a human model of the gastrointestinal tract and showed beneficial effects on their intestinal microflora. This GM goat milk could be left at room temperature for at least two days, which is especially important in developing countries (Wall et al., 2009, CAST Issue paper 43, Animal Agriculture's Future through Biotechnology, Part 8).

Transgenic cattle for increased casein production were created in New Zealand. The cheese produced with this GM milk had increased levels of essential amino acids and bioactive peptides. Transgenic goat expressing a special enzyme in its milk, which converts long-chain saturated fatty acids to their monounsaturated forms, was created by scientists in California. Consumption of this milk that has higher proportions of monounsaturated fatty acids might have a beneficial effect on human cardiovascular health. A transgenic rabbit model was created in the Agricultural Biotechnology Center, Gödöllô, Hungary to illustrate the unique ability of transgenic technology to provide novel foods tailored for specific dietary requirements of sufferers of the genetic disorder phenylketonuria, who cannot metabolise the amino acid phenylalanine. In this study, a low-phenylalanine, mutant rabbit K-casein was expressed at high levels in milk and this modified K-casein could be purified by simple one-step purification (Laible, 2009, Comparative Immunology, Microbiology \& Infectious Diseases, 32: 123). Concerns for food safety initiated generation of transgenic cows and goats, which do not produce prion protein, the causative agent of bovine spongiform encephalopathy (BSE). These animals are resistant to BSE. In those cases transgenic founder animals were created by cloning, therefore public judgement is more dismissive.

\section{Reduced environmental footprint}

Livestock transgenesis could contribute to sustainable agriculture with the intention to reduce pollution and more effectively utilize both feed and animal resources. To address the problem of manure-based environmental pollution in the intensive pork industry, the phytase transgenic pig called Enviropig was developed by Canadian scientists at the University of Guelp. The saliva of these pigs contains the enzyme phytase, which allows the pigs to digest phytate, the most abundant plant-derived source of phosphorus in the pig diet. Without this enzyme, phytate passes undigested into manure to become the most important manure pollutant of pork production. This genetic modification reduces the excretion of undigested phosphorus in faeces by $30-60 \%$, which could ameliorate surface water eutrophication and the environmental footprint of phytase production as food supplement (Golovan, 2001, Nature Biotechnology, 19: 741). In 2010, Canada approved limited production of EnviroPigs ${ }^{\mathrm{TM}}$ in controlled research environments. It will be years before meat from genetically engineered pigs could be available for human consumption (Figure 8.3.). 


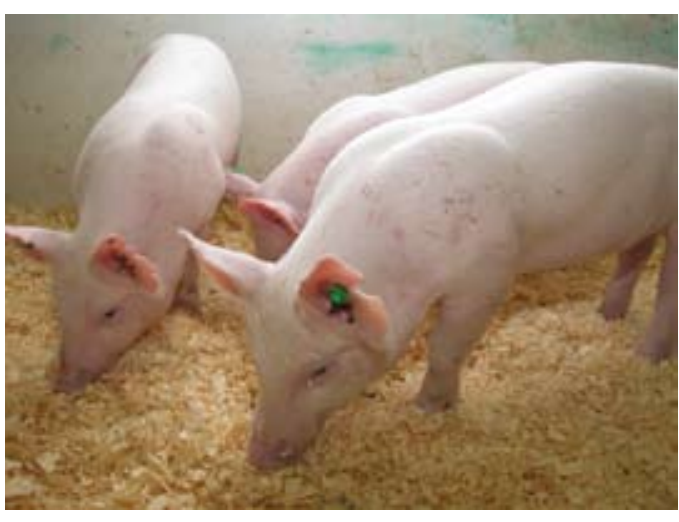

Figure 8.3. Female pigs from the transgenic Cassie line of EnviroPig ${ }^{\mathrm{TM}}$. Photo by Prof. Cecil Forsberg (University of Guelph, Canada)

Ecotoxicological studies resulted transgenic fish which produce special reporter proteins upon environmental stress. The protein produced is usually a fluorescent protein of jellyfish origin. Different variants have been developed, some responding to general environmental stress, others to heavy metal or aromatic compound pollution. Scientists at the Department of Aquaculture of SZIE, Gödöllô, Hungary adopted this technology.

\section{Controversial applications}

Like any novel approach, the transgenic technology also has the potential to be used for aims unacceptable for ethical or ideological reasons. Some of these applications arose from important basic research but resuscitate as pure business, which is decorous for the scientific community.

Green fluorescent protein and several other variants are currently being used in live-cell imaging. Transgenic zebrafish were developed which produce bright bioluminescence in the aquarium. Although originally these animals were not developed for the ornamental fish trade, they are among the first genetically modified animals to become publicly available as pets. Other transgenic pets (dogs, cats) with reduced allergen potential for their owners are also being sold. Industry has started to use some transgenic animal models queried by society. Transgenic goat lines were established, which produce spider silk in their milk. Spider silk is one of the strongest and most flexible protein fibres. It is planned to be used in the monocoque body of Formula-1 cars and in bullet-proof shirts. Another herd of transgenic goats was generated to produce, in the milk, a special enzyme which is able to detoxify organic phosphates. Originally the goats were produced for biomedical purposes, but later on military applications became dominant, to provide protection against nerve gases. This application generated extensive argument.

\section{Conclusions}

Recombinant proteins of pharmaceutical value produced by GM livestock animals have already reached the market, and the number of these products is expected to increase in the forthcoming years. The number of valuable large GM animals as models of human diseases or as sources of xenografts is also steadily increasing. On the other hand, particularly in the EU, significant politico-socioeconomical barriers are to be overcome before some of the biotechnological solutions in animal production are adopted. It is worth recalling that artificial insemination, which is now common practice both in agriculture and in human medicine, was initially also regarded as unacceptable. 


\section{GM plants for feeding livestocks}

\section{JÁNOS GUNDEL}

The scientific community has invested decades of research to study where and how biotechnology could be expediently applied, first of all to increase the feed base. Equally pertinent in deciding how to use available options is the question whether or not such intervention would result in a deterioration of quality of life, environmental damage or pollution or any other unforeseeable adverse consequences. While increasing the feed base, we should simultaneously improve feed quality, increase nutrient contents and improve chemical composition. Supplying the world's population with food of animal origin, together with the accompanying increase in demand for both quantity and quality represents one of today's most pressing problems. Therefore it is necessary to introduce and use technologies, which would ensure sustainability, taking into account the far-reaching impacts of using such methods on environmental protection and the production of various foods. Already as early as the 1980s, earlier possibilities for genetic modification such as cross-breeding, selection and mutation-breeding were complemented by methods of gene technology.

An interesting fact is that, while the number of Western European publications dealing with gene technology and basic and applied research in the past 30 years has been higher than that seen in North America, the actual production of GM feed crops in Europe is considerably less significant than across the Atlantic Ocean.

GM activities related to feed production may be separated into three major disciplines: microbiology, plant genetics and, more recently, animal genetics, which have set the goal of the production of transgenic lines (varieties) (e.g. through gene introduction). The resulting varieties are fundamentally influenced or, at times, even altered radically by certain feeding practices.

The scientific results related to GM plants utilized in food and feed production practices are also divided into three areas. The first of these is the new feed crops (especially soybean and corn, but also wheat, canola, sugar beet and potatoes), the second is feed additives (e.g. enzymes) and the third group is the group of medicines. The latter two are not addressed in this article, because they are regulated under completely different areas of authority. However, this last group includes, for example, the various growth hormones (BST, PST) and other products created by gene technology, including antibiotics (and other medicines), and other new products (developed in order to be able to replace the old ones), which play an important role in animal feeding and, consequently, in human food production (including food safety).

It is a fact that the perception and acceptance of GM crops are affected by the extent of biological and biotechnological knowledge of consumers and by how well they are actually informed about what they eat. Consumers may be either insufficiently informed about GM-related food issues or may have received information which was inadequately presented or not sufficiently clear. The development of science is much faster than the expansion of the knowledge of the average consumer, and this is equally true for some experts.
In the future, the competition in the production of animal-derived food and in the processing industry in Europe will depend on the inventiveness of plant genomics and biotechnology and, of course, their applications. The prevention or mitigation of the adverse consequences of climate change and the development of renewable energy sources should necessarily lead to an appreciation of the role of GM feed. In Hungary, the current legislation restricting the cultivation of GM varieties (ban) causes a serious competitive disadvantage for Hungarian farmers. The opinion of the vast majority of domestic experts is that Hungary should adopt the practice of the EU, by changing the legal framework on matters such as domestic GM legislation, since there is no sound scientific, environmental, and economic justification for depriving Hungarian farmers of the right to produce GM food and feed plants and to make use of the economic benefits from such activities

According to some arguments, already in 2007 the area of fields sown with GM crops showed a yearly increase of approx. $12 \%$, with totals in some locations reaching up to $90 \%$. This growth has been continuous in the last 10 years and there is no sign of reaching the plateau in the near future. The EU imports a great deal of GM feed, mainly soybean and corn. The approval process for GMOs in the United States takes about 15 months, whereas similar processes in the EU can take even years. This disparity places the competitive ability of food/feed production and the related agribusiness sectors in great jeopardy. If the licensing procedure does not change in the EU (the zero-tolerance rule remains valid), the consequences will be dramatic. Experts predict that $44 \%$ of the poultry sector and $35 \%$ of the pig sector could be permanently lost, because there is no practical, affordable alternative to the 35 million tons of soybean imports

The first generation of GM feeds comprises varieties and species of food and feed plants of global importance such as soybean, corn, canola and cotton. These are seed feeds produced by plants that may be grown either partially or completely free of pesticide and herbicide or, in some cases, insecticide use.

The second generation of GM feeds are expected to be important for livestock because of their improved nutrient content or ratio (e.g amino acids, fatty acids and vitamins), their ability to better utilize nutrients, or their reduced contents of harmful substances (lignin, phytin and allergens), a result of molecular breeding made to improve their genetic make-up.

Comparison of the first generation of GM crops to their isogenic lines or to non-GM feed crops showed insignificant differences in nutrient composition (Table 9.1.) 


\begin{tabular}{|c|c|c|c|c|c|c|}
\hline \multirow{2}{*}{ Composition } & \multicolumn{2}{|c|}{ Maize } & \multicolumn{2}{|c|}{ Maize } & \multicolumn{2}{|c|}{ Sugar beet } \\
\hline & Isogen & $\underset{\text { resistant }}{\text { Insect }}$ & Isogen & $\underset{\text { Herbicide }}{\text { tolerant }}$ & Isogen & $\underset{\text { tolerant }}{\text { Herbicide }}$ \\
\hline \multicolumn{7}{|c|}{ Nutrients, (g/kg DM) } \\
\hline Crude protein & 108 & 98 & 120 & 119 & 72 & 60 \\
\hline Crude fat & 54 & 56 & 31 & 35 & 3 & 4 \\
\hline Crude fiber & 23 & 25 & 34 & 30 & 56 & 46 \\
\hline Crude ash & 15 & 16 & 19 & 18 & 30 & 30 \\
\hline $\begin{array}{l}\text { N-free } \\
\text { extracts }\end{array}$ & 800 & 805 & 796 & 798 & 839 & 860 \\
\hline Starch & 710 & 708 & 692 & 701 & n.d. & n.d. \\
\hline Sugar & n.d. & n.d. & n.d. & n.d. & 736 & 744 \\
\hline \multicolumn{7}{|c|}{ Amino acids, (g/kg DM) } \\
\hline Lysine & 2,9 & 3,0 & 3,3 & 3,2 & n.d. & n.d. \\
\hline Methionine & 2,2 & 2,1 & 2,6 & 2,5 & n.d. & n.d. \\
\hline \multicolumn{7}{|c|}{ Fatty acids ( $\%$ of total fatty acids) } \\
\hline Palmitic acid & 12,4 & 12,5 & 11,5 & 11,8 & n.d. & n.d. \\
\hline Oleic acid & 31,1 & 28,6 & 27,7 & 27,4 & n.d. & n.d. \\
\hline Linoleic acid & 50,0 & 51,2 & 57,0 & 56,3 & n.d. & n.d. \\
\hline
\end{tabular}

n.d. $=$ no data

Table 9.1. Selected constituents of transgenic insect resistant (Bt) and herbicide tolerant maize grains and herbicide tolerant sugar beet (PAT) in comparison with isogenic lines (based on data from literature)

American researchers analyzed grain and corn silage from GM (Roundup Ready [herbicide resistant]) and traditional plants for two consecutive years, and found that the difference between the two years was greater than the difference between GM and non-GM variants. Namely, in the second year, irrespective of the GM treatment, the protein, $\mathrm{Ca}$ and $\mathrm{P}$ contents of the grain were higher than in the first year. These data, in the authors' view, represent clear evidence that there is no correlation between the gene modification and nutrient content.

In Bt corn, the risk of Fusarium infection can be decreased by the corn's resistance to the corn borer; therefore, this type of GM corn produces higher quality feed. Italian researchers used young piglets in feeding experiments to compare isogenic corn and Bt corn. The isogenic corn plants were more infected by Fusarium toxin (100\%) than the control plants (Fumonisin B1: 36\%; Deoxynivalenol: 86\%). The differences were also reflected in rearing outcomes (final weights were 22.0 and $22.6 \mathrm{~kg}$ and the average daily weight gains were 375 and $396 \mathrm{~g}$, respectively).

Feeding trials were carried out in different countries on poultry using Bt corn and soya, and again no significant differences were found between the effects of isogenic and transgenic feed.

In 2005, German researchers reported a feeding experiment in which 10 generations of Japanese quail were fed with $40-50 \%$ isogenic or transgenic (Bt176) maize in the feed without observing any significant differences in the animal weight gains or states of health. The meat and egg quality and hatchability of eggs were the same and the DNA fractions of organs and body fluids also revealed no detectable differences.

Experiments carried out on ruminants - cows, beef cattle and sheep - to investigate milk production or fattening found no differences, regardless of whether maize of isogenic or transgenic origin was fed.

American scientists reported feeding experiments on dairy cows using two types of silage made from Bt maize (one had a shorter vegetation period than the other). Regardless of the genotype or the length of the vegetation period, no effect was shown either on rumen fermentation or milk production. In another experiment Bt maize silage was compared to conventional corn silage; the daily portion contained $40 \%$ silage, $28 \%$ coarse cornmeal of the same origin, $10 \%$ alfalfa haylage and $22 \%$ concentrate (extracted soybean meal, feed-fat, minerals and vitamin mixture) with a $17.5 \%$ crude protein content. Silages made of GM or control maize were tested on rumen fistulated cows. No significant difference was found between cows that had consumed these maize variants either in milk production $(28.8 \mathrm{~kg} / \mathrm{day})$, or in the chemical composition of their milk, or in dry matter intake $(22.8 \mathrm{~kg} / \mathrm{day})$, or even in the indicators of rumen fermentation ( $\mathrm{pH}$, volatile fatty acid content and ratio, NDF digestion).

In metabolic and fattening experiments carried out on pigs, a ratio of $70 \%$ insect resistant corn (Bt) or herbicide-tolerant corn (RR) or a control of non-GM corn was given as feed. There was no difference either in nutrition value or in fattening indices. The foreign DNA was followed using a PCR technique and, in harmony with earlier findings, it was no longer detectable in the product destined for human consumption. In an experiment using similar methodology, feed containing transgenic soy was fed to pigs and identical results were obtained.

In the second generation of GM crops the chemical composition of the plants is altered in different ways. These changes can increase the protein content (including the amino acid composition) and fats (including the constituent fatty acids) and/or their relative proportions. Additionally, the starch content may also increase, but utilization of various minerals, vitamins and other compounds may be improved. There are attempts to reduce the proportion of indigestible parts in feed plants, for example, of lignin or of the cell wall components, although this 
alteration may increase the vulnerability of the cell wall, leading to a decrease in pest resistance. Increased production of certain enzymes (e.g. phytase) may result in reduced amounts of anti-nutritive materials, e.g. glucosinolates or alkaloids.

Phytic acid in cereals numbers among the most significant non-nutritional materials which, in addition to inhibiting P-digestibility in monogastric animals, greatly reduces $\mathrm{Ca}, \mathrm{Zn}$ and $\mathrm{Fe}$ as well as protein utilization. This, in turn, is of dual importance, both for meeting the phosphorus requirement of the animals and for mitigating the environmental impact by reducing the amount of nonutilized phosphorus excreted. The phytase gene from Aspergillus niger has been introduced into phytin phosphate containing cereals; the plants thus generated are also second generation GMOs. According to the literature, this enzyme is normally synthesized in some feeds, but phytase level can also be increased in further plant species rich in phytin phosphate through gene transfer (e.g. in cereals, rapeseed, soybean or even alfalfa). In wheat experiments, it was found that phytase is synthesized in the seed endosperm, namely during seed filling. In this manner, the phytase activity can increase up to four times the normal level and will remain largely stable. When using such a feed, the amount of phosphorus in the manure will be significantly reduced, i.e. the phytase enzyme supplement may become unnecessary in swine and poultry rations.

A summary of the work of German researchers on the potential feeding value of forage made of the first and second generation of GM plants is shown in Table 9.2.

\begin{tabular}{|c|c|c|}
\hline \multirow{2}{*}{ Parameters } & \multicolumn{2}{|c|}{ GMO generations } \\
\hline & 1. & 2. \\
\hline Determinations of important constituents & & \\
\hline $\begin{array}{l}\text { - crude nutrients } \\
\text { - genetically modified nutrients (e.g. amino acids, fatty acids, } \\
\text { vitamins, enzymes, etc.) } \\
\text { - genetically modified undesirable substances (e.g. plant } \\
\text { constituents such as lignin, inhibitors, glycosides, etc., or } \\
\text { secondary substances, such as mycotoxins, pesticides, etc.) }\end{array}$ & $\begin{array}{l}+ \\
- \\
+ \\
+\end{array}$ & $\begin{array}{l}++ \\
++\$ \\
++\$\end{array}$ \\
\hline $\begin{array}{l}\text { Digestibility, nutrient transformations, availability of modified } \\
\text { nutrients in the target animal species }\end{array}$ & $(+)$ & ++ \\
\hline In vitro studies on the assessment of nutritional value & $(+)$ & $(+)$ \\
\hline $\begin{array}{l}\text { Feeding experiments with special/categories of target animal } \\
\text { - performance of animals and quality of foods of animal origin } \\
\text { - animal health }\end{array}$ & $\begin{array}{l}(+) \\
(+)\end{array}$ & $\begin{array}{l}++ \\
(+)\end{array}$ \\
\hline
\end{tabular}

Meaning of symbols: -- not necessary; $(+)$ may be advantageous; + recommended; ++ necessary;

$\S$ for modified components

Table 9.2. Proposal for the nutritional assessment of GM feeds

(on the basis of various literary sources)
As concerns the first generation of GMOs, a number of animal experiments and investigations have revealed that, from a nutritional point of view, there is no significant difference between transgenic and isogenic plants. This topic has been discussed in a very large number of communications, and in none of these can one find an instance in which a researcher refers to, even in long-term experiments, any disability or other negative outcome when compared to isogenic feed. In 2006, German scientists published an opinion stating that they felt free to argue that the GMO content in animal feed does not affect either product quality or nutritional value (the difference is always possibly due to other causes, such as the degree of mycotoxin contamination or that animals fell ill for other reasons during an experiment).

In the future, a wider spread of the second generation of GMOs is expected, especially in those cases where the amount of beneficial (desirable) components is increased and that of the disadvantageous ingredients - antinutrient materials - is reduced. In Table 9.3., various possibilities for genetic modification are presented.

\begin{tabular}{|c|c|}
\hline Crop & Variable features \\
\hline Alfalfa & + phytase; + resveratrol; lignin $\uparrow$ \\
\hline $\begin{array}{l}\text { Canola } \\
\text { (rape) }\end{array}$ & $\begin{array}{l}\text { Vitamin } E \uparrow ; \text { lauric acid } \uparrow ; \gamma \text {-linoleic } \uparrow ;+\omega-3 \text { fatty acid; }+\beta \text {-carotene; } \\
\text { 8:0 and 10:0 fatty acids } \downarrow ; \text { medium chain fatty acids } \uparrow\end{array}$ \\
\hline $\begin{array}{l}\text { Manioc } \\
\text { (cassava) }\end{array}$ & cyanogenic glycosides $\downarrow$ \\
\hline Lupine & methionine $\uparrow$ \\
\hline Maize & $\begin{array}{l}\text { methionine } \uparrow \text {; fumonisin } \downarrow \text {; insect resistance; Vitamin } C \uparrow ; \text { protein } \\
\text { with favorable amino acid profile } \uparrow \text {; sulphur-containing amino acids } \uparrow\end{array}$ \\
\hline Potato & $\begin{array}{l}\text { starch } \uparrow ; \text { very-high-amylose starch } \uparrow ; \text { inuline molecules } \uparrow ; \\
\text { sulphur-rich protein } \uparrow ; \text { solanine }\end{array}$ \\
\hline Rice & $+\beta$-carotene; iron $\uparrow$; allergenic protein $\downarrow$ \\
\hline Sorghum & improved digestibility of nutrients \\
\hline Soybean & $\begin{array}{l}\text { improved amino acid composition; increased sulphur-containing } \\
\text { amino acids; oleic acids } \uparrow \text {; immunodominant allergens } \downarrow\end{array}$ \\
\hline Sweet potato & protein content $\uparrow$ \\
\hline Wheat & glutenins $\uparrow$ \\
\hline
\end{tabular}

Table 9.3. Examples of GM crops with nutritionally improved traits intended to provide benefits to consumers and domestic animals 
The presented literature survey shows that there is a large interest in breeding of new GM crops (feeds), in developing new features and in their exploitation. There are a large number of publications, none of which refers to any post-consumption health deterioration due to GM plants. Although there are concerns about and fear of GM crops, the vast majority of the publications take the view that feeds containing GM plants do not unfavourably affect the quality of animal products or their value in human nutrition.

\section{The possible role of GM technology in the breeding, propagation and processing of future biomass crops}

LÁSZLÓ MÁRTON, MIKLÓS GÁBOR FÁRI

\section{Introduction}

Interest towards biomass crops (after the beginning of the oil era) dates back to the 1970s, when the first oil crisis hit the oil and carbon energy-dependent modern world.

The recent interest is mainly due to environmental considerations in addition to the increase of oil prices and lessening resources. The role of $\mathrm{CO}_{2}$ emission accompanying the use of fossil fuels in increasing atmospheric $\mathrm{CO}_{2}$ concentration is obvious, and its environmental consequences in the climate are also clearly detectable (diminishing ice caps and glaciers, increase in the temperature of oceans, dying coral reefs, increasing sea level etc.).

In geological terms, there is no time for introducing positive changes aimed at stopping or reversing the negative trend. One generation from now, $40-50 \%$ more people should be fed and provided with energy, whereas (if we do not make every effort) the average surface temperature could increase by $2-6 \mathrm{C}^{\circ}$, which could have dramatic effects such as desertification and a significant reduction and relocation/realignment of the currently used agricultural lands. By maximum use of green energy sources (wind, water, flux and reflux of rivers, solar cells or even nuclear energy) $\mathrm{CO}_{2}$ emission could be significantly reduced; however, that would not be enough to stop the current trends, and increased fixation and fossilization of $\mathrm{CO}_{2}$ are also necessary. The most straightforward method for this is by photosynthesis of plants. Field crop production is based mainly on annual plants, which does not reduce the $\mathrm{CO}_{2}$ level, as the intensive soil cultivation mobilizes more $\mathrm{CO}_{2}$ than is fixed. Unfortunately, in order to avoid food shortage the current range of agricultural crops cannot be changed much. However, if this greenest energy transformation, namely photosynthesis is used, and it is done with perennial plants where the above-surface parts can serve as energy sources, while the below-surface parts significantly contribute to organic matter (carbon) accumulation in the soil, thereby providing a negative carbon balance, significant atmospheric carbon depletion can be achieved. Plant photosynthesis is the most efficient process for $\mathrm{CO}_{2}$ removal, which is fuelled by 150000 TW solar energy hitting the surface of the Earth every year. Presently, we use 15 TW energy yearly, which may reach 20 TW by 2030 .

The series of energy conversions in photosynthesis are intensively studied not only for $\mathrm{CO}_{2}$ fixation and biomass production, which is a central area, but also for more direct utilizations such as direct electricity and $\mathrm{H}_{2}$ production (natural fuel cells) in artificial and semi-artificial systems as well as for direct production of liquid fuels. 
In the present transitional period, all opportunities must be seized to start positive trends and to avoid an environmental catastrophe. The use of GM technologies cannot be given up for some sentimental principles, since via its use the sustainable approach of the "green" era can be made more diverse and more effective, and giving it up would mean that we give up the most effective, scientifically-proven and rapidly applicable tool for food production and energy production based on green biomass

\section{The ratio of available marginal lands and the summary of current} technologies

In 2010, environmental and agricultural experts determined that there are about 320-702 million hectares of so-called marginal areas in Europe, the USA, Africa, India and South-America, where agricultural production was abandoned or whose fertility is so low that they are unsuitable for profitable agricultural production in their present state. If the steppes, savannas and natural bushy areas (so-called LIHD areas) are added to the marginal lands, then they total 1.107-1.411 million hectares. In theory, agricultural fuel production based on biomass in these lands (using traditional crops and agro-techniques) could produce $26-55 \%$ of the world's liquid fuel demand. Responsible professionals and non-professionals agree that the natural resources accumulated during millions of years cannot be further destroyed, not even in the present intensive era of energy demand.

As a result of the above theoretical calculations, agro-economists drew the attention of the scientific world to the fact that the further demolition of natural vegetation can only be avoided if the world's energy requirement is produced on only the 320-702 million hectares of marginal agro-ecosystems. For this purpose, as stated by American scientists, the most effective biological, genetic and other breeding methods should be used and new and adequate agricultural cultivation and biomass processing methods should be developed. The prediction is that breeding, propagation and growing of the future biomass and energy crops in the next fifty years will be done by applying a combination of the most modern techniques, among which the GM method should also be used.

\section{What should be the future biomass and/or energy crops like?}

There is a severe technical problem in biomass crop production. Plant breeding has a history of 10.000 years, but so far the most frequent target has been the fruit or other specific plant parts, and the aim has been to obtain the highest amount of the utilized part from the lowest possible amount of biomass. With respect to energy, these parts represent a negligible amount, as plants store the chemical energy from $\mathrm{CO}_{2}$ fixation, reduction in the form of carbon chains, mainly in the form of cellulose-lignin-hemicellulose in the cell walls. Therefore, if the objective is energy transformation ("energy gain"), we should focus on the biomass, where energy is stored, which means mainly the transformation, i.e. release of the energy of the C-C bonds in the cell walls. In most of the current crops, the ligno-cellulose cell wall material is only a by-product (straw, stem, seed coat, processing waste), which is usually expensive due to the costs of collection and transport.

The contribution of biomass to the energy use in Hungary could be increased up to $80 \%$ from the present $10 \%$ if the suitable lands were used for biomass crop production.

The so-called third generation biomass crops were selected and bred for the purpose of intensive and environmentally-benign biomass crop production. As it cannot be avoided that biomass production should be limited to lands of marginal quality and should not compete with food production, drought tolerance or its opposite, tolerance of inundation and waterlogging can be an important aspect. Crops should be resistant to other extreme growing site conditions such as high salt content, extreme $\mathrm{pH}$, chemical residues, heavy metal pollution, which do not allow for food crop production, or the crops grown there are not suitable for consumption. Only perennial crops can be taken into consideration, because the annual soil cultivation reduces $\mathrm{CO}_{2}$ fixation, and the perennial herbaceous crops show considerable, continuous sustainability, can be of remediative/ameliorative nature (especially grasses) and can improve the lands of marginal quality.

\section{How can GM techniques assist the sustainability of biomass industry?}

The significance of GM technology and its contribution to the breeding of optimum biomass crops and to their optimization for energy production can be enormous, because an extremely quick and effective breeding work is necessary due to the shortage of time. This justifies the use of new genetic-epigenetic modification/controlling GM and the somaclonal breeding techniques. The advanced state of recombinant DNA technologies enables an increasingly precise transformation of the genome and the fine tuning and adjustment of gene functions, with which the lack of century-long breeding work can be compensated

\section{Major R\&D fields}

For the use of GM technology, not only the easily and spectacularly changeable parameters should be considered such as the augmentation of fermentable sugar and starch content, the modification of starch structure and metabolism optimal for fermentation, where otherwise great advances have been made during the effort towards producing seed alcohol more efficiently.

Revolutionary successes have been achieved, when complex metabolic changes with a dramatic effect on cell wall composition and structure were created in GM biomass crops. On the one hand, a higher energy level was achieved by increasing e.g. the lignin content, which results in surplus energy on direct burning and pyrolysis. On the other hand, significant results were achieved in increasing the proportion of cellulose and hemi-cellulose, which are the substrates of fermentable sugar-release (chemical or enzymatic decomposition). These can 
be used for the production of ethanol by yeast fermentation, or of alcohols with longer carbon chains and various other products by other microbes. The significance of these longer carbon chained products is that they can be separated from water without the energy-consuming distillation, they can be easily purified and directly used as liquid fuel. At present the highest efficacy of ethanol extraction is achieved in steam-combustion systems producing $1 \mathrm{t}$ alcohol from 4 tons of dry plant matter, i.e. $10 \mathrm{t}$ alcohol per hectare for a local giant reed (Arundo donax L.) plantation (Figure 10.1.), which can reach up to 25-35 tons of ethanol under tropical and subtropical conditions. This is several times higher than the efficacy of seed alcohol or even sugarcane alcohol production. These data refer to the so-called "basic type" plants; it is not difficult to calculate how dramatic the impact of the introduction of new-generation GM plants on the ethanol market will be.

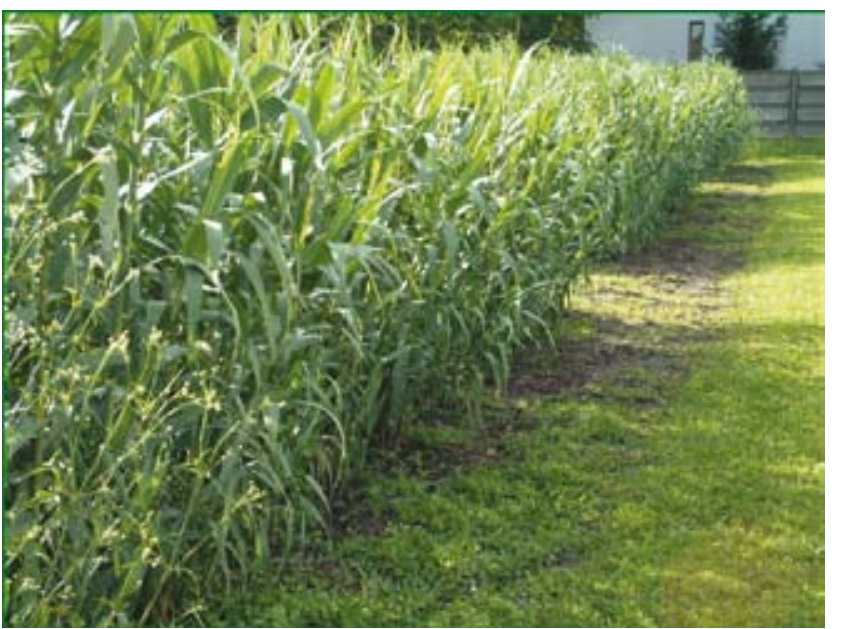

Figure 10.1. One of the most promising area of the modern plant biotechnology is understanding the complex physiological and molecular regulation processes of somatic embryogenesis. Four-monthold giant reed (Arundo donax L.) syn-plant biomass plantation propagated from somatic embryos (Plants for the Future Experimental Garden, University of Debrecen, 2010)

Also very promising is the creation of GM plants, in which not only the accessibility and composition of the cell wall are changed, but the cellulosedecomposing enzyme is also produced by the plant, an enzyme which, under the appropriate conditions, can decompose the plant material to sugars. In other cases, the expensive decomposing enzymes were synthesized by plants and were added to the decomposing reaction. The excess "syngas" derived from pyrolysis of GM crops with higher lignin content and of lignin by-products, which is used directly for heating and electricity production, can be polymerized and converted into liquid fuel by modern variants of the old Fischer-Tropsch method.
Another field where the GM technology has made great contributions is the increase in crop size (since size and growth rate are of primary importance in biomass crop production). Giant sized plants were grown by modifying the regulation of the genes controlling hormone production and effect (e.g. ARGOS) and genes controlling cell division. The shift in the cell wall composition in GM crops resulted not only in more or less products (shift in the lignin/cellulose ratio), but also e.g. in better digestibility, which indicates a better availability of cellulose and hemicellulose for degradation to sugars.

The application of the so-called siRNA technology in GMOs started a revolution in the field of biomass energy. The significance of this technology in the regulation of metabolic processes and their dramatic transformation is comparable to that of the introduction of restriction endonucleases in recombinant DNA technology. In this way, multigenic functions can be regulated, blocked or adjusted as needed.

There has not yet been a breakthrough, but intensive research is carried out on the modification of photosynthesis mechanism in $\mathrm{C} 3$ and $\mathrm{C} 4$ plants, so that optimum water utilization could be reached in biomass energy crops. This is an extremely complex process, and there are still a lot of questions to be clarified at the level of basic research. The siRNA technology will definitely result in a revolution in this field, not the least because there are several multigenic functions in these processes.

As most of the biomass crops (GM or non-GM) are not suitable for further classical plant breeding (e.g. they are sterile, have no seeds or they are so aneuploid that they cannot be handled by sexual propagation), the only options are somaclonal breeding and clonal propagation. Prolonged clonal propagation can be frequently accompanied by clonal deterioration, which can be explained on the one hand by the accumulation of epigenetic changes and, on the other hand, by virus accumulation. However, in continuously maintained embryogenic cell cultures and plants regenerated therefrom, great homogeneity and stability can be observed, therefore if such cultures are used for producing GM plants and then mass micropropagation procedures are developed, the above disadvantages can be prevented. Clonal (epigenetic) ageing can be avoided and continuously rejuvenated plants can be planted.

Another alternative is classical vegetative propagation, which can be done only expensively or with difficulty in the case of large plants with rhizomes by chopping up of rhizomes or stem cuttings, and very frequently it does not yield viable propagation material (e.g. there is no viable bud on it, or it is virus-infected that can be transferred to other plants).

The road to producing future biomass crops will probably lead through programming the genes responsible for embryogenesis by using GM techniques, as it has been successfully done for certain model species. In such cases, the GM technique is an option but not always an essential method (Figure 10.2). 


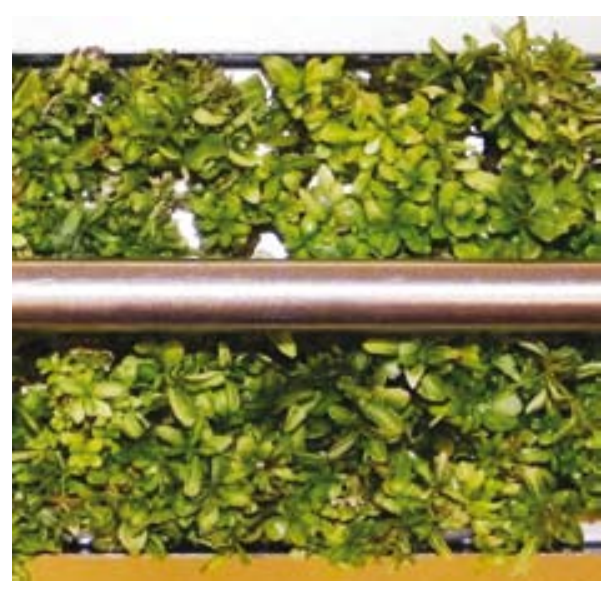

Figure 10.2. The Artificial Plant Ovary (APO) developed by the University of Debrecen will generate promising technological break-throw in the large-scale clonal propagation practice of some GM-based industrial biomass crops (Populus sp, Eucalyptus sp., sugarcane, etc.)

A new propagation technology developed in recent years based on international co-operation and on an instrument, APO (Artificial Plant Ovary) developed by the Plant Biotechnology Department of the University of Debrecen enabled the mass propagation of Arundo donax, one of the most promising biomass crops, which produces record amounts of biomass under almost any climate condition. One of the special biological keys of the process was the controlled induction and maintenance of the continuous embryogenic state, whereas the other was the synchronized, homogeneous regeneration of such cultures into syn-plants. These new biotechnological tools resulted in a production system which meets all the requirements of large-scale production, the testing of which has started worldwide. This procedure can also be applied to other biomass crops; the elaboration of similar procedures is in progress e.g. for Malvaceae at the University of Debrecen.

\section{Biopesticides and biofertilizers}

\section{LÁSZLÓ HORNOK, KATALIN POSTA}

Biopesticides are products containing a microorganism, i.e. a bacterium, fungus, protozoan or virus as active ingredient. These compounds can be used to control a variety of plant pathogens or pests, but a particular biocontrol organism itself is in most cases specific to the target organism. Biofertilizers are preparations that utilize microorganisms to enrich soil fertility; the activity of microbes present in a biofertilizer may also result in increased levels of nutrients for plants.

There are several advantages to using such products. Biopesticides (i) are, by nature, less harmful than chemical pesticides, (ii) have a narrow range of action compared to chemical pesticides that may affect non-target organisms, (iii) are non-persistent, (iv) help to avoid environmental problems associated with chemical pesticides, and (v) can be safely used in IPM (integrated pest management) programs. Biofertilizers (i) are low-cost products, (ii) pose no danger to the environment, (iii) enrich the soil and (iv) support long-term sustainability. However, the widespread use of biopesticides and biofertilizers is limited as they do not yield rapid, spectacular results. Furthermore, these products are highly sensitive to environmental factors and/or the application technology.

\section{Major biocontrol and biofertilizer agents}

The most widely used biopesticides utilize the entomopathogenic bacterium Bacillus thüringiensis. Various strains of this bacterium produce different protein mixtures and specifically kill larvae of one or a few related insect species. Some B. thüringiensis strains control larvae of herbivorous moth species, while others are specific for larvae of beetles, again others for mosquitoes, etc. The target insect species are determined by whether the protein produced by a particular B. thüringiensis strain can bind to a gut receptor of larvae and is activated by enzymes of the digestive tract. Biocontrol fungi are also used against insects, but on a much smaller scale and mostly in the glasshouse. Verticillium lecanii is commercially used in Europe against aphids and white flies. Metarhizium anisopliae and Beauveria bassiana are utilized to control larvae of beetles, including Colorado beetle and codling moths. Commercial products, like Tick-EX G, Tick-EX EC based on M. anispoliae spores as active ingredient are available in the US. The use of baculoviruses as insect specific control tools has also been attempted. These viruses are ingested by the insect larvae, leading to their infection and death in 5-8 days

Agrobacterium tumefaciens, the causal agent of crown gall disease of a wide range of ornamental, fruit and vegetable plants is successfully controlled by A. radiobacter strain K84, producer of the bacteriocin, agrocin 84 .

Hyperparasitic fungi, natural antagonists of plant pathogenic fungi can also be used in IPM technologies. Biopreparations containing Ampelomyces quisqualis, Coniothyrium minitans, Pythium oligandrum, as well as several species of 
Gliocladium and Trichoderma are commercialized in North-America, Europe, Israel and the Far-East. The hypovirus-infected hypovirulent strains of Cryphonectria parasitica are also widely used against aggressive isolates of the fungus causing chestnut blight in Europe and North-America.

Biological control of weeds by fungal pathogens is a promising way of combating unwanted, mainly exotic or alien plant species invading arable lands. The basic requirement for a successful mycoherbicide is target-specificity of the biocontrol agent without risk of extension of host range. Among the best known examples are Phytophthora palmivora and Colletotrichum gloeosporioides used against milky weed in Citrus plantations and round-leaved mallow (Malva pusilla), respectively. Commercial mycoherbicides are available both in Europe and North America, but their use is restricted to orchard crops in specific distances from annual crops to avoid extension of the infection.

As regards biofertilizers, nitrogen biofertilizers (containing N-fixing bacteria, like Azotobacter, Azospirillum, Rhizobium and Shinorhizobium) help increase the nitrogen level of the soil; phosphorus biofertilizers (like mycorrhizal fungi, mainly Glomus spp. or bacteria, like Bacillus spp.) enrich the P-content of the soil, whereas plant growth promoting rhizobacteria (PGPR) improve the growth of plants through mobilizing various micronutrients and out-competing plant pathogens. Azotobacter and Azospirillum spp. are free-living and associatively living nitrogen fixing organisms, respectively, and are used in a wide range of crops including cereals, vegetables and ornamental plants. The bacterial strains used in commercial biofertilizer products are improved strains obtained by selection or induced mutation and have increased competitive capability or resistance to chemical pesticides, or a combination of these desired traits. Rhizobium (Azorhizobium, Bradyrhizobium, Shinorhizobium) species form a stable symbiotic interaction with legume plants and fix nitrogen while living in root nodules developed by the host plant as a result of the nodulation activity of the bacterial partner.

\section{Use of GEMs (genetically engineered microorganisms) for biocontrol} and biofertilization

Biological preparations are generally regarded as being safer than their chemical counterparts. However, they often tend to be less efficient and more unpredictable in action, which limits their widespread use. One of the measures by which the efficiency and manageability of these products can be improved is the use of genetically engineered microorganisms (GEMs) as active ingredients. GEMs are microorganisms modified by introducing a gene or genes using recombinant DNA technology. GEMs can have traits and functions that their natural ascendants can never produce.

There are many examples for the successful experimental use of GEMs as biopesticides and biofertilizers; their application on a commercial scale is, however, limited to a few cases.

The killing process by wild-type baculoviruses is very low, preventing the widespread use of these organisms. However, genetically engineered viruses that carry toxin gene(s) whose product(s) are specific to the target insect have a much higher killing efficiency. Such transgenic preparations ( $H z$ NPV, Se NPV) have been commercialized in the US and used against Heliothis and Spodoptera spp. in cotton, grapes, ornamentals and vegetables. Scorpion genes were used to create a hypervirulent fungus that can kill specific insect pests, including beetles that destroy coffee crops without the need to release this biocontrol agent into the natural environment. Several B. thüringiensis strains modified by recombinant DNA techniques have been registered in the US (CRYMAX ${ }^{\mathrm{TM}}$, Lepinox $^{\mathrm{TM}}$, Raven ${ }^{\mathrm{TM}}$ ): these GEM strains contain either a specific $c r y$ (insecticidal protein encoding) gene combination or modified/chimeric cry gene constructs, resulting in superior toxicity to the target organisms. Furthermore, a sitespecific recombination approach has been used to delete all foreign DNA elements from the recombinant cry plasmids after introducing them into the recipient $B$. thüringiensis strains, making these GEMs practically non-transgenic. Another way of utilizing the superb activity of $B$. thüringiensis was the transfer of the BT-toxin gene into Clavibacter xyli to control corn ear worm.

Agrocin 84 produced by $A$. radiobacter K84 is highly efficient against $A$. tumefaciens. However, the bacteriocin that confers the activity of the biocontrol agent is encoded on a plasmid along with resistance genes to agrocin 84 . The plasmid could be transferred to pathogenic $A$. tumefaciens strains under natural conditions, causing loss of efficiency of this biocontrol method. The problem was overcome by deletion of the tra region: the GEM biocontrol agent, available commercially under the marketing name "No Gall" became incapable of conjugative transfer of agrocin resistance to pathogenic strains. Gliocladium and Trichoderma strains, improved in cell-wall degrading activity and/or pesticide tolerance have also been engineered and a chitinase encoding gene from Serratia marcescens was transferred into Pseudomonas fluorescens to control fungal diseases.

Bacillus mucilaginosus, the silicate bacterium utilized as a multifunctional biofertilizer dissolves insoluble potassium, phosphorus and other beneficial elements. NKTS-3, the transgenic strain of this bacterium containing a phytase expression cassette has increased extracellular phytase activity, liberates inorganic phosphorous in the soils and improves phosphorus acquisition of roots. Genetically modified Rhizobium strains fix more nitrogen owing to the increased levels of expression of the nitrogenase gene cluster, nifHDK. Such strains have been approved to be tested in field experiments in Mexico. The nitrogen fixing activity of nodules induced by genetically engineered $R$. leguminosarum with high catalase activity was increased by 1.7 to 2.3 times.

\section{Concerns about the release of GEMs}

Due to the high reproduction capacity of microorganisms and the particular ways of genetic exchange in microbe populations, public concerns about GEMs are much stronger than those about GMO plants or animals. Due to the limited 
market acceptance of GEM, large biotechnology companies are tending to withdraw from the field. Indeed, horizontal gene transfer is a commonplace in bacterial genera containing both human and animal pathogens, and such transfer may also occur between bacteria and fungi. GEMs with new fitness traits may be more resistant against the competitive organisms of their wild-type parents, and therefore their release may cause perturbations in the natural microflora. Furthermore, using gene constructs bearing antibiotic resistance genes may result in unexpected and undesirable gene escape. On the other hand, un-controlled gene transfer can be prevented by genetic engineering (an example was mentioned above for $A$. radiobacter), and sequences coding for antibiotic resistance can be destroyed in transgenic organisms (like in GE B. thüringiensis). GEMs engineered to have improved anti-microbial activity may exert non-target effects on natural microbial communities, but other measures, like crop monoculture, ploughing or pesticide treatments may certainly cause much stronger adverse effects.

Despite concerns, countries with rapidly growing economies like China, India or the US have less aversion to the introduction of GEM-based biopesticides and biofertilizers. Irrespective of practical applications, research on such organisms is carried on in other parts of the world, as methods, gene constructs, products and technologies developed while working with GEMs are utilized very efficiently in industrial microbiology and molecular plant breeding.

\section{Further reading}

- Cummings, J. (2003): GM microbes invade North America. Science in Society, 19: 39 .

- Garay-Munoz, C. et al. (2010): Mode of action of Bacillus thüringiensisgenetically modified Cry1AbMod and Cry1AcMod toxins: role of alkaline pH in toxin oligomerization. Southwestern Entomologist, 35: 383-386.

- Hirsch, P. E. (2005): Release of transgenic bacterial inoculants - rhizobia as a case study. Plant and Soil, 266: 1-10.

- Hunter, P. (2009): Fight fire with fire. Can biopesticides fill the void left by banning chemical pesticides and herbicides. EMBO Reports, 10: 433-436.

- Thomas, M. B., Read, A. F. (2007): Fungal bioinsecticide with a sting. Nature Biotechnology, 25: 1367-1368.

\section{The role of GM microbes in the fermentation technology}

\section{KORNÉL KOVÁCS}

Biomass stores the energy captured from sunlight during photosynthesis in the form of chemical bonds holding the molecules together. In the various ways of producing renewable biofuels, this chemical energy is released and converted into a form that is easy to utilize in everyday energy consuming devices, e.g. transportation vehicles, light bulbs etc. The most commonly used fractions of biomass are the ones prepared for energy storage purposes by the photosynthetic organisms themselves. These are sugars or their polymers, such as starch or cellulose.

The transformation of starch into sugar is an important branch of the starch industry and one of the most important applications of biotechnology. Countless foods contain ingredients produced by the breakdown of starch. Enzymes are the key to these chemical reactions - enzymes that are predominantly produced with the help of genetically modified microorganisms. Starches are chemically bound clusters of sugar molecules found in plants. Under the right conditions, starch molecules can be broken down into sugar. Sugar is the most preferred carbon and energy source for almost all microbes used in biotechnological processes. This process makes it possible to obtain sugar from the starch of many different plants, rather than just sugar beets or sugar cane. This is now being done by industrial-scale starch saccharification. The most important sources of starch are maize, potatoes, and wheat.

Strong acids were once used to break apart starch molecules and release sugar. Now, enzymes do the job offering many advantages: with enzymes, the process targets the proper chemical bonds much more precisely. Different enzymes can be used to produce syrups with different levels of sweetness and different technical characteristics. The end products are not only used as custom tailored ingredients in countless foods and drinks, they can also be further processed into glucose, artificial sweeteners, or fat substitutes.

For a long time, enzymatic breakdown of starch (saccharification) did not make economic sense. Things changed, however, as soon as the enzymes responsible for this process became available at low cost, high quality, and in practically unlimited quantities. Now, almost all of the enzymes used to break down starch are produced with the help of genetically modified microorganisms.

Plants are used as a starch source. A certain portion of the raw material may be genetically modified. Cultivars of maize and potato have been produced, in which the structural properties of the starch molecules are altered so that saccharification takes place more efficiently. These starch sources are then used in bioenergy production, making subsequent fermentation of the sugar component more efficient and the overall process economically more viable. Next generation renewable energy carriers will utilize lignocellulosic raw material. Lignocellulose 
is the material produced in the largest amount on Earth. However, due to its structural complexity direct utilization of this biomass by microbes is extremely low. Ongoing research aims at the production of efficient enzymes in large quantities that would make the utilization of lignocelluloses for energy production purposes economically feasible. These so called third generation energy carriers made from lignocelluloses, like ethanol, butanol or biomethane, biohydrogen, will make possible to stop the mass utilization of fossil fuels, which poison our environment and are rapidly depleted.

The production of energy is achieved via direct utilization of solar energy by phototrophic organisms or through dark fermentative conversion of biomass to valuable energy carriers. Photosynthetic microbes such as algae, cyanobacteria and phototrophic bacteria can be genetically modified so that their energy and/ or metabolic pathways are directed towards the production of useful molecules. A recent example for this strategy is the production of isobutyraldehyde by a genetically modified cyanobacterium. Cyanobacteria and algae carry out photosynthesis and using this energy source they build up their molecules from carbon dioxide, the major contributor to global warming and associated environmental problems. The genes of four enzymes, obtained from other bacteria, were incorporated into the genome of Synechococcus elongatus, a thoroughly studied cyanobacterium. As a result, a new biosynthetic pathway was engineered in the host cyanobacterium, which produces isobutyraldehyde from the $\mathrm{CO}_{2}$ fixed. The product is volatile, thus its removal from the cyanobacterial culture is fairly easy. This compound is the precursor of several useful chemicals, including isobutanol, which has a great potential as fuel alternative to gasoline.

Among the alternative energy carriers hydrogen appears to be the most promising, because it burns to environmentally friendly water when utilized, and may be transported and stored rather easily. Hydrogen can be produced in biological processes: in algae and cyanobacteria solar energy captured by the photosynthetic apparatus is converted into chemical energy through water splitting, the reaction that yields oxygen and can also produce hydrogen. Upon utilization, these components are combined to form water and energy is released in a cycle driven by the Sun, a practically unlimited and safe energy source.

The understanding of molecular fundamentals of hydrogen production and utilization in microbes is a goal of supreme importance both for basic and applied research applications. The key enzyme in biological hydrogen metabolism is hydrogenase, which catalyses the formation or decomposition of the simplest molecule occurring in biology: molecular hydrogen. The simple-looking task is solved by a sophisticated molecular mechanism. Hydrogenase is a metalloenzyme, harbouring $\mathrm{Fe}$ or sometimes $\mathrm{Ni}$ and $\mathrm{Fe}$ atoms. Like most metalloenzymes, hydrogenases are extremely sensitive to inactivation by oxygen, high temperature and other environmental factors. These properties are not favourable for several potential biotechnological applications. In metal-containing biological catalysts it is the protein matrix surrounding the metal centres that provides the unique environment for the $\mathrm{Fe}$ and $\mathrm{Ni}$ atoms which allows hydrogenases to function properly, selectively and effectively. Therefore, a major goal of hydrogenase ba- sic research is to understand the protein-metal interaction. The problem is not simple to address, as some of the methods for scientific investigation provide information on the metal atoms themselves without directly observing the protein matrix around them. Other modern techniques at our disposal reveal details of the protein core, but do not display the metal centres within. A combination of the various molecular approaches is expected to uncover the fine molecular details of the catalytic action of metalloenzymes. Engineering the protein matrix by random and site-directed mutagenesis, expression of the enzymes in various hosts lead to genetically modified stable hydrogen producers. In addition, through metabolic engineering, i.e. switching genes the protein products of which take part in various energy production or consumption pathways, allow the guidance of energy towards hydrogen production (Figure 12.1)

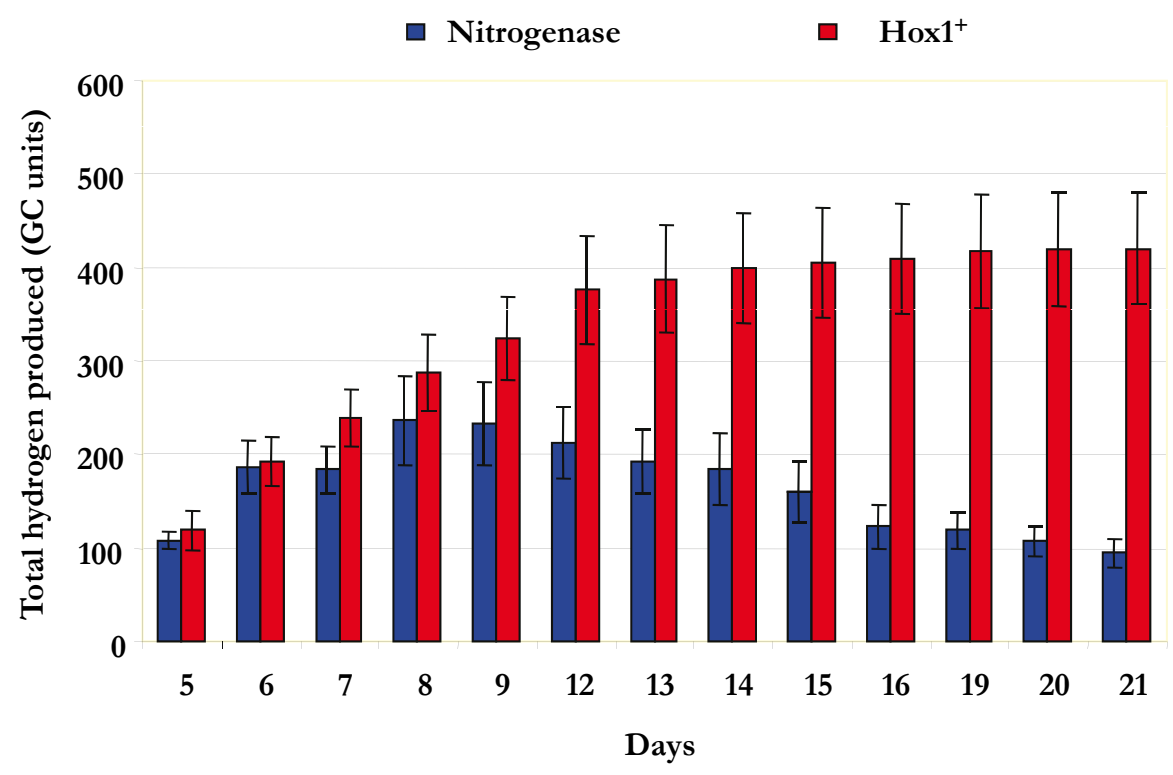

Figure 12.1. Hydrogen production by a phototrophic bacterium (Thiocapsa roseopersicina). The blue columns indicate the daily hydrogen production of the wild-type strain. A GM mutant (red columns) is capable to produce more hydrogen for a longer period of time using an altered metabolic route

Dark fermentation of biomass usually involves anaerobic biotechnology due to its potential to produce value-added products from low-value feedstock such as waste streams. In addition, it provides an opportunity for the removal of pollutants from liquid and solid waste more economically than other processes. Genetically altered metabolic routes give improved yields in the production of 
biohydrogen, biomethane, biobutanol, bioethanol and biodiesel in anaerobic conversion of biomass to useful energy carriers. A rapidly developing area is butanol production. Certain Clostridia are capable of butanol synthesis during the so-called solventogenesis stage of their growth. During solventogenesis these bacteria predominantly produce a mixture of butanol, ethanol, acetone and some organic acids. In order to make the bacteria focus on butanol production, the numerous alternative pathways present in Clostridia should be blocked by knocking out the genes coding for some key enzymes in those pathways.

In the field of food production and preservation another group of anaerobic bacteria, the lactic acid bacteria (LAB) are used widely. Among other foodstuffs, cheese is made by them. Cheese is made from the protein-rich fraction of the milk. The main protein component in milk is casein, a hydrophobic protein stabilized in the aqueous environment by another protein called Kappa-casein. In order to recover casein from the milk, Kappa-casein should be removed, and this job is done by an enzyme named rennet or chymosin. Rennet has been traditionally obtained from the stomach of calves: it degrades proteins and thus upon addition to the milk it decomposes Kappa-casein leaving the hydrophobic casein behind, which precipitates and forms the curd. As large scale cheese production emerged in the 1960s, there was a shortage of rennet. Microorganisms have been modified genetically to yield chymosin that is identical to the enzyme obtained from animals. This can be used to produce better quality cheese than the original rennet. The first scientists to make chymosin in this way in 1981 used bacteria; chymosin is now obtained from yeasts. In 1988, chymosin was the first enzyme from a genetically-modified source to gain approval for use in food industry. These proteins behave in exactly the same way as calf chymosin, but their activity is more predictable and they have fewer impurities. Today about $90 \%$ of the cheese industry products are made using chymosin from genetically-modified microbes. It was relatively easy to accept the GM enzyme for cheese production, because the producing GM microbe is not present in the food production system, only the excreted chymosin, which is indistinguishable from the calf stomach version. In addition, the enzyme itself is also broken down during the cheese maturation process.

LAB have a long history of use by man, they are naturally present in raw food material and in the human gastro-intestinal tract. LAB are widely used as starter cultures for fermentation in the dairy, meat and other food industries. These food-grade bacteria can also improve the safety, shelf life, nutritional value, flavour and quality of the product. Moreover, LAB can be used as cell factories for the production of food additives and aroma compounds. It is further assumed that LAB may function as probiotics and contribute to the general health of the consumer. The uncontrolled genetic alterations of LAB that occur during random mutagenesis may lead to strains with altered properties. The level of such mutations depends on several environmental factors, e.g., radiation, mutagenic compounds and growth conditions. Selection of strains that have been subjected to uncontrolled genetic modifications is used to improve certain characteristics of the fermented end product, like flavour, structure, nutritional value. For example, a random mutant Lactobacillus strain was isolated that was defective in lactate production, but had an increased level of the butter flavour compound diacetyl. LAB can also be selected for removal of undesirable compounds from raw food materials. In traditional yoghurt fermentation lactose is degraded only partially. One of the products is galactose, which may be harmful for people suffering from galactosemia. With laborious screening a galactose-fermenting spontaneous mutant Streptococcus strain was isolated, which solved this problem.

An alternative to random mutagenesis is targeted modification of the DNA. Genetic engineering offers a range of manipulations from single base pair substitutions, mutations, insertions of genes into the chromosome to deletion of portions DNA resulting in inactivation of specific enzymes. Food fermentation at large scale may suffer from bacteriophage infections resulting in lysis of the starter culture. The transformation of industrially important strains with phage resistance genes for other LAB could generate phage resistant strains. Another important feature of food products is texture. Gene clusters encoding exopolysaccharide producing enzymes have been transferred from one LAB strain to the other. The genetically modified strains improved the viscosity and texture of the fermented product.

As far as risk is concerned, it should be noted that despite the fact that the strains obtained via random mutagenesis are not considered GMOs, the occurrence of predictable unintended genetic alterations is very likely to take place within such mutant strains as well. The potential event of horizontal gene transfer following expression of foreign genes in LAB used in food fermentations is one of the major concerns that affect the safety assessment for consumer and environment. When the applied genetic elements originate from LAB that has a long and safe history of use in food, essentially no new risks are introduced. 


\section{Genetically modified plants as the basis of food quality improvement and biopharmaceutical production}

\section{LÁSZLÓ TAMÁS, MÁRIA OSZVALD}

Plant breeding has been practiced for thousands of years to improve the quality of crops. It was based on selection using the try and error approach. Due to the increasing demand of a stable supply of high-quality food crops, research stations have been founded in many countries, like in Hungary since the middle of the $19^{\text {th }}$ century. Breeding was carried out using advanced scientific methods and equipment to ensure the best possible results. Apart from the classical crossing, a new methodical approach in plant breeding has been introduced, based on gene technology. By this method tailor-made modifications of certain characteristics of the crop have become feasible. During this process a gene or a group of genes are inserted into the genetic material of a high-quality crop that lacks the particular properties the new DNA fragment codes for. The introduced DNA fragment may come from the genetic material of the same species but from a different variety (wheat-wheat), from a closely related species (wheat-rice), from another plant family (wheat-amaranth), or from a completely different organism (wheat-bacteria).

The first genetically modified plants carried new features (e.g. resistance to herbicide, pathogens or insects) to stabilise the yield by decreasing the damage on crops. In the last two decades gene technology has also focused on other aspects of breeding, such as improving the quality of food crops. It has become possible to reach this goal, because a wide range of information has been gathered on the biochemical pathways and on the properties of different types of molecules capable of influencing the quality of processed food. Applying this quality-based knowledge as well as the results of gene/protein expression and regulation studies, quality-driven modification of plants and also of food products is now a realistic issue.

The new species produced by the gene technology approach are advantageous for consumers/society by producing

1. healthier food products for maintaining health or preventing diseases

2. suitable products for patients suffering from allergies

3. nutritionally improved food products providing more balanced/healthy

4. products with a more appealing and marketable appearance that require less energy during the producing process

5. fruits/vegetables with improved/longer shelf life resulting in less waste
The first promising result in quality improvement research was the transgenic tomato that could be stored for long periods. This transgenic tomato called Flavr Savr was declared by the responsible US authority, the Food and Drug Administration "as safe as the traditionally produced tomato" after a thorough evaluation in 1994. It was therefore permitted to be sold on the market. The taste of the tomato was improved by traditional breeding, but it did not become popular, because it still lacked certain features. One of the advantages of this tomato species was the reduced energy requirement during the production process. Due to the lower cost of processing, the puree from this type of tomato was $20 \%$ cheaper compared to that made of other tomatoes. It was also available in Europe, for instance in the UK, between 1996 and 1999, but later it was called back from the market.

Bread is one of the most important wheat flour products in Hungary and in other European countries. Its quality is characterised by e.g. loaf volume, elasticity, firmness, shelf life etc. and to a large extent determined by the quality of flour milled from the starchy storage organ, called endosperm tissue. The quality of wheat grain and the flour is characterised in many ways. For millers, seed hardness is an important feature, whereas bakers are more concerned with the functional properties (water absorption capacity, bread making parameters) of the flour. Bakers can alter the functional properties of the flour by various additives/improvers; however, due to a change in the attitude of society around the turn of the millennium, supplementation has lost a lot of its desirability.

Good quality flour, which capable of satisfying the needs and requirements of the consumer without additives can be also milled from genetically modified wheat grains. The factors affecting the mixing properties of the dough have been studied in detail, hence most of them are well-known. Proteins required for the production of good quality (e.g. high loaf volume) bread were determined and characterised. Studies on proteins in the flour showed that increasing the proportion of a particular one, called HMW (High Molecular Weight) glutenin subunit in wheat flour resulted in stronger dough and better quality bread. The results derived from non-transgenic wheat flours were confirmed by experiments on flours milled from genetically modified wheat lines. GM wheat lines were developed in the beginning of nineties and have been studied extensively in the last 15 years. Hungarian scientists also took part in this international effort. They proved, among others, that blending poor quality flour with GM flour resulted in a blend suitable for premium quality bread production. According to the carefully designed studies carried out in the last decade, it was possible to produce transgenic wheat lines which were practically equivalent with non-GM wheat at the level of analysis provided by modern laboratory technologies (Figure 13.1.). 
L88-31

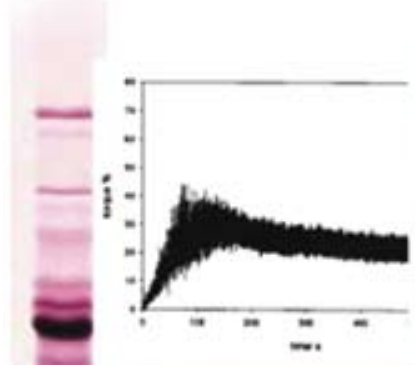

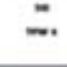

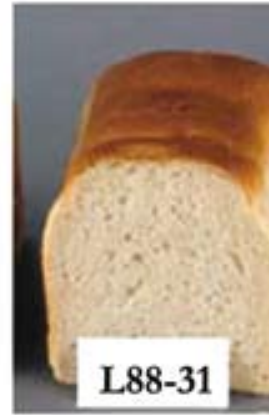

$3118 \mathrm{ml}$
B102-1-2

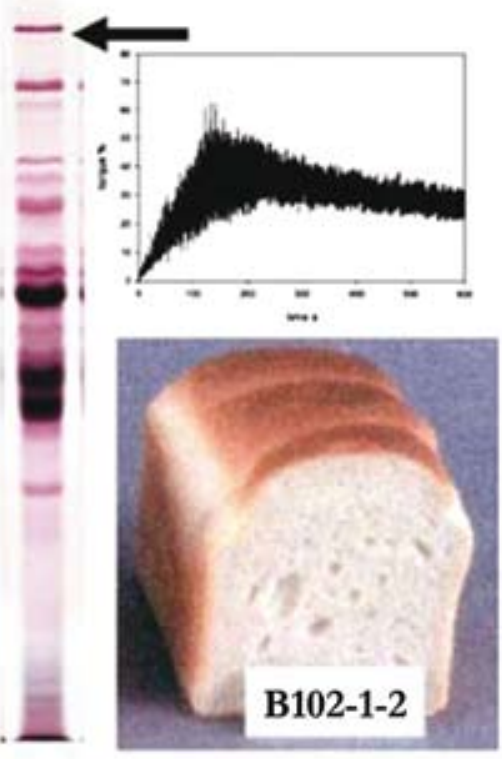

$3183 \mathrm{ml}$
Figure 13.1. Increased amount of the wheat Ax1 HMW glutenin subunit has positive effects on bread making quality of the wheat flour. Loaves baked from transgenic (102-1-2) line had higher volume than wild type (L88-31) flour. Protein expressed in the transgenic line indicated by arrow in the electrophoretogram of the proteins. Mixograph curves (in frame) registered during mixing are different, indicating stronger (more elastic) dough produced from transgenic wheat. (Shewry, P. R. et al., 2008, Plant Biotechnology: Transgenic Crops. Food Biotechnology Book Series: Advances in Biochemical Engineering/ Biotechnology, 111: 149)

Although wheat is considered to be a staple food, its consumption may be risky for a large number of people sensitive to it. It has turned out that more and more people suffer from different types of allergies caused by wheat flour. One of the most widely known allergies is the celiac disease. Another group of wheat allergies is called wheat sensitivity, which affects around $10-15 \%$ of the global population. Good records of scientific evidence proved that wheat allergies are caused by the protein in the flour. It has to be noted that not all of the proteins are allergenic, and different patients show sensitivity to different groups of allergenic proteins. People sensitive to these proteins should avoid eating wheat products. However, this is a significant challenge, because not too many alternative wheat-free products are available on the market and, importantly, all of these are too expensive.
Rice and maize seed proteins are less allergenic, if at all, for most people and are considered to be safe for those suffering in wheat allergies. Unfortunately, it is difficult or almost impossible to produce even poor quality bread or similar baking products using rice or maize flour. It is due to the lack of the special proteins capable of forming the appropriate structure in wheat dough. Several options have already been studied to serve the problems of patients with wheat allergy and one of them is through genetic modification. As a result of modification, these cereals are able to synthesize one or more carefully chosen wheat proteins in the storage organ of the grain. This new flour is suitable for dough formation, although with low quality. Products made of GM rice flour should provide a solution for a group of sufferers not sensitive to the particular proteins introduced into this GM rice. More GM rice lines would mean more groups of sensitive people whose quality of life could be improved.

The goal of the studies that have been carried out in Hungary was to characterise the GM rice lines developed by the introduction of a wheat HMW glutenin subunit gene. The functional quality of the flour has been tested and dough mixing experiments were also performed. Beyond these tasks, the allergenic effects of the modified flours are going to be investigated.

One sixth of the world's population suffers from hunger, which equates to more than one billion people. Managing to feed this population is therefore a major challenge of our time. In addition, according to the FAO, over half of the global population are affected by different forms of food deficiencies. This so called "hidden hunger" is due to the quality rather than the quantity of the food consumed by poor people. In developing countries people living on staple crops of low nutritional quality have unbalanced diets that supply neither the energy, nor the protein, nor the micronutrients required for a healthy life. Sufficient and balanced diets can be achieved if not only the protein quantity but also its amino acid content is improved and provides the appropriate amount of vitamins, lipids, antioxidants and edible fibres. This remains, however, out of reach for a large proportion of the world's population due to economic factors and the lack of suitable food crop varieties. One possible solution could be to supplement staple food with industrial products. An even better solution, however, would be if the excess amount of essential micronutrients were synthesized by the crops. This is a uniquely sustainable solution, as crops could be grown locally. This process, aimed at increasing the nutritional quality of staple food is called biofortification. Biofortification can be done either through conventional, or gene technology assisted breeding. Biofortification offers great promise for not only those billions of undernourished people living in remote areas that are unable to afford nutritious food, but also for those who live in developed countries. A few examples are shortly reviewed below regarding the use of transgenic crops with improved nutritional quality.

Nearly two billion people are deficient in zinc. In children this condition causes infection and diarrhoea, and as a consequence around 800,000 children are lost worldwide per year. According to the WHO, iron deficiency is also a common nutritional disorder, causing many pregnant women and preschool children 
to suffer from anaemia causing increased risk of morbidity and reduced work productivity. Studies carried out on transgenic plants carrying a special enzyme to hydrolyse the microelement binding protein in the seed have proved the advantage of this approach: a positive correlation was observed between lower protein content and the bioavailability of minerals. Growing GM crops could therefore substantially reduce the number of deaths around the world.

Although the protein content of the food consumed appears to be adequate in many cases, the amino acid composition is inappropriate. This may well be due to the fact that a single cereal contributes the major part of people's protein intake. Protein nutritional quality is determined by the ratio of essential amino acids, which cannot be synthesized by humans and must be supplied by the food. Because cereal grains contain low levels of essential amino acids, their nutritional quality is poor. Genes coding for high-quality proteins used for biofortification of cereals usually come from seed proteins of dicots, as they have higher proportions of essential amino acids. Studies aimed at improving the nutritional quality of wheat flour have also been carried out in Hungary. The gene that was introduced into the wheat genome codes for a protein recommended by the WHO as a highly nutritional protein. This protein, called AmA1, is derived from amaranth (Amaranthus hypochondriacus) and is expressed only in the starchy storage organ of the grain in GM wheat. It was concluded that not only the essential amino acid content of the flour but also the functional quality of the flour can be improved, if the introduced protein is carefully chosen.

Vitamin A deficiency is estimated to affect one third of children under the age of five around the world. It annually claims the lives of around 600,000 children under the age of five, and 250,000-500,000 children become blind each year owing to vitamin A deficiency. One solution to improve the health of those affected is to supplement their diet with synthetic products. Plants, however, are also able to synthesize and deposit vitamins in edible storage organs in large quantities. There are several advantages to using gene technology to improve the vitamin content of crops. Firstly, the active stereoisomer is produced only in the plant, secondly the energy requirement in the plant bioreactor is provided by natural resources. It has been demonstrated that the biosynthetic pathway for beta-carotene production can be established exclusively in the storage organ of the rice grain through gene technology. The transgenic line "Golden Rice" is named after the colour of the polished rice seeds, provided by the high concentration of the yellow pigment. A new line with improved quality, named "Golden Rice 2" has also been developed, producing 23 times more provitamin A. Although both were produced as a humanitarian tool and could be used as a biofortified food in locations where there is a shortage of dietary vitamin A, none of them are available as a food crop (Figure 13.2.)

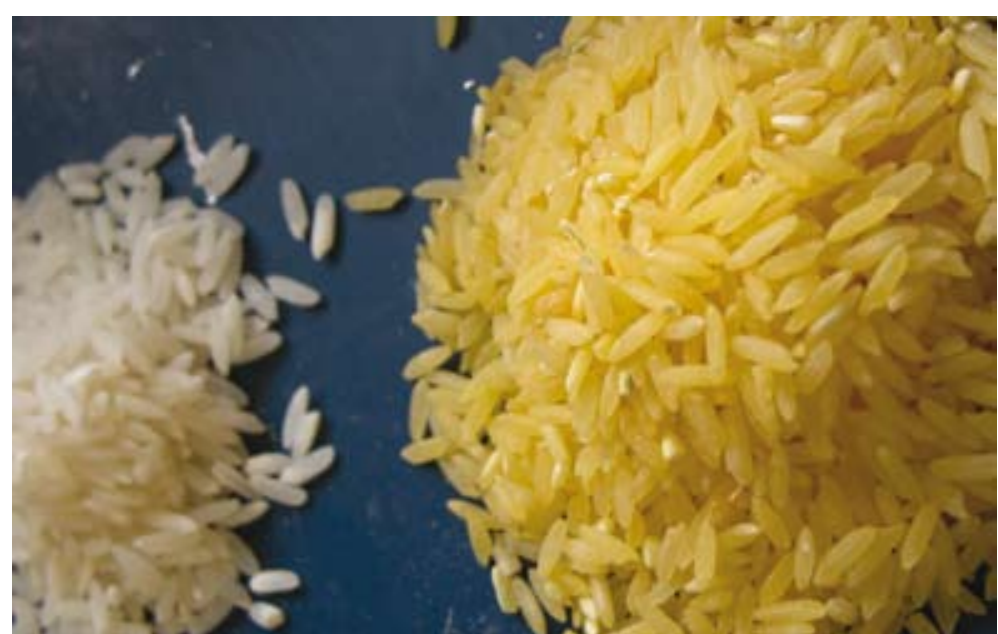

Figure 13.2. Wild type (white) and transgenic (yellow) rice seeds

Plants are not only a source of food and industrial material but some can also be used as medicines to treat different illnesses. Apart from native species, genetically modified plant lines could also become good resources of high-value compounds in the near future to be used against diseases or to maintain health. The pharmaceutical industry uses specially designed bacteria, fungi and animal cell cultures in large containers (fermenters) under controlled environments for long periods of time to produce useful molecules. Plants, as ideal bioreactors, are also able to synthesize molecules required by the industry. Through genetic engineering it is possible to introduce specific genes into the genetic material of plants' nucleus or chloroplast, enabling us to specify exactly what molecules the plant should synthesize. Because chloroplasts are large in number within a plant cell, a substantially higher amount of the foreign protein is produced if the gene of interest is integrated into the chloroplast rather than the nuclear genome. The molecules produced by these GM plants have many applications, examples of which include: research/diagnostic reagents, high grade proteins (trypsin), monoclonal antibodies (immunoglobulin) and edible vaccines (providing immunity to particular diseases). GM plants developed for the biopharmaceutical industry would have several advantages. For example, they have a lower negative impact on the environment compared to fermenters, since they utilise renewable resources (e.g. solar energy, rain and soil) and do not produce waste water. Recombinant protein synthesized in the seed crop would remain stable for long periods at ambient temperature without loss of activity. Furthermore, vaccine expressed in edible tissue needs no extraction or purification, and has an extremely low risk of contamination by human pathogens. The needle-free delivery of the edible vaccine further reduces the risk of contamination.

The very first plant to be used for biopharmaceutical production was the tobacco plant, as genetic modification was simple and the plant was easily 
grown in the greenhouse. However, due to the problems related to tobacco plants, other species are considered instead, such as bananas, potatoes, sugarcane, corn, peas etc. It is desirable that the foreign high-value compounds be synthesized in sufficiently large quantities in the storage organ, and that the plant material be easily harvested and stored. The first plant-based biopharmaceutical product was human growth hormone reported in 1986. Since then several thousand articles have been published on more than one hundred therapeutic molecules produced by various plant species. These high quality, high value molecules can be classified as hormones, immunoglobulins, interferons, interleukins, human and animal growth factors, human autoantigens, vaccines against bacteria and viruses, and more. Several plant-made biopharmaceuticals produced either in the chloroplast or in the cytoplasm of the plant cell have been involved in clinical trials, some of which are advancing beyond the first phase.

Experiments aimed to produce edible vaccines in cereals have been carried out in Hungary. Small fragments of different bacterial proteins considered to be non-toxic were synthesized in transgenic rice lines. It has been proved that the recombinant protein antigens are produced exclusively in the starchy storage organ of the rice grain. The level of protein expression is sufficient to generate appropriate amounts of antibodies against $E$. coli or cholera after the consumption of only a few grams of transgenic seed. In summary, the GM rice lines developed could be used as a rice seed based edible vaccine.

\section{Summary}

Transgenic crops used for biofortification are no magic bullet, but they may prove to be one of the most cost-effective ways to reduce hidden hunger by using food that people already grow locally and eat.

\section{An overview of the economic impacts of GM crops}

\section{JÓZSEF POPP, NORBERT POTORI}

In the long term, the world's rapidly growing food requirement can only be met through the sustainable use of resources, the availability of which is limited. Agriculture has to manage cultivatable land and use the scarce water and fossil energy resources much more carefully and efficiently in the future, and, in many areas, discharge significantly less artificial fertilisers and chemicals to the environment than at present. Clearly, a return to archaic farming methods and farm structures is not an option but, according to the views of the authors of a joint study published in January 2010 by the French National Institute for Agricultural Research (INRA) and the International Agricultural Research Centre for Development (CIRAD), there is need for the wide-ranging application of innovative technologies, and for social changes.

From among the innovative technologies available the cultivation of genetically modified (GM) plants is of particular significance. The number of transgenic events (vector constructions) in commercialised GM crops worldwide has grown rapidly in recent years, and is expected to triple in the first half of this decade to around 120 in 2015. Amongst industrial crops, new hybrids with new qualitative characteristics such as improved frost and cold tolerance, salt and drought tolerance, virus resistance, resistance to fungal pathogens, delayed ripening, as well as the production of human or animal proteins or enzymes etc. will come onto the market in the near future.

\section{Global status}

The produce of GM plants and its derivatives (whether or not mixed with non-GM stocks) account for, year on year, a greater volume of international trade and constitute an increasing share of the world's feed and food chains. The International Service for the Acquisition of Agri-biotech Applications (ISAAA) estimated that in 2009 in 25 countries worldwide some 14 million farmers planted commercialised GM varieties and that 57 countries granted regulatory approvals for the use of biotech crops. The global area planted to GM crop varieties amounted to 134 million hectares, which represented $9 \%$ of global arable land. GM plants covered 61.5 million hectares in the developing countries, amongst which the most important producers of biotech crops were Argentina, Brazil, India, China, Paraguay and South Africa (Figure 14.1.) 


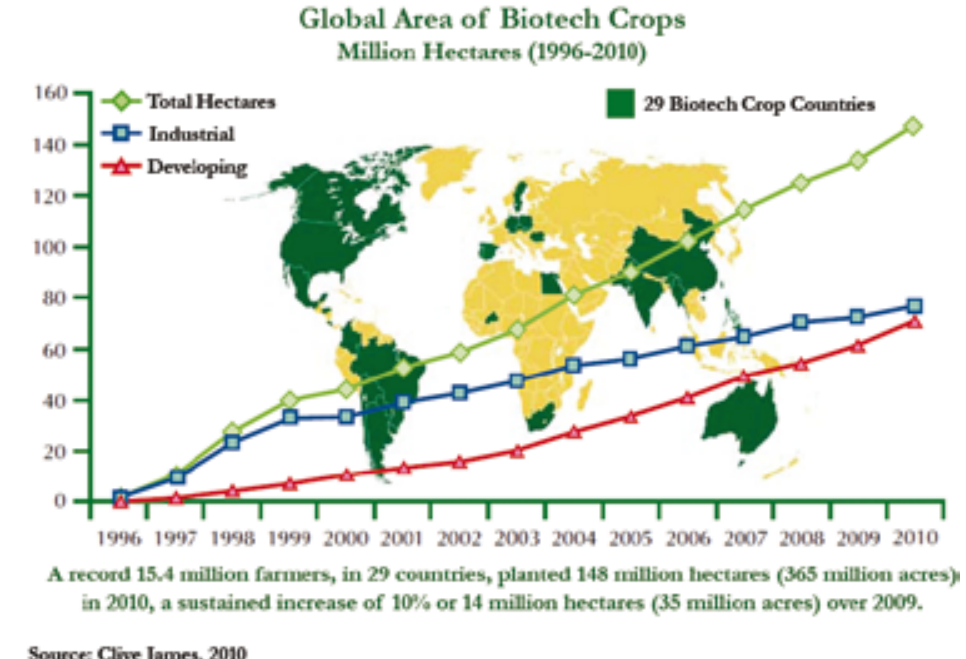

Figure 14.1. World trends of GM crop production show continuous increase

Amongst biotech crops in 2009, GM soybeans occupied the largest area with 69 million hectares, equal to $77 \%$ of the global soybean plantings. In the second place, with 41 million hectares, stood GM maize which had a $26 \%$ share of the total maize plantings. This was followed by GM cotton and GM canola with 16 million and 7 million hectares, respectively, representing 49 and $21 \%$ shares of global plantings. Herbicide tolerant and insect resistant biotech crops covered 83.6 and 21.7 million hectares, respectively, whereas double or triple stacked hybrids were already planted on 28.7 million hectares. In the United States and Argentina GM varieties were grown on 91 and $98 \%$, respectively, of the growing area in 2009. Even though the cultivation of GM varieties was officially approved only in $2005,71 \%$ of the land used for soybean production in Brazil was sown with GM seeds. $85 \%$ of the maize area in the United States and 50\% in Argentina was sown with GM varieties in 2009. Although the Brazilian government did not permit the cultivation of MON810 maize before February 2008, the area sown with GM varieties reached 30\% in the case of summer maize and 53\% in the case of winter maize (safrinha) in 2009. Even more noteworthy is that in the world's leading canola exporter country, Canada, GM canola in the same year accounted for $93 \%$ of the sowing area.

The only biotech crop grown in the European Union is the MON810 insect resistant (Bt) maize. Although the production of the herbicide tolerant T25 maize is also permitted, it has not been introduced onto market. MON810 occupied 94,850 hectares $(1.1 \%$ of the entire maize area) in 2009 , representing a $12 \%$ decrease on 2008 and a $14 \%$ decrease compared to 2007 . The Member States growing GM maize in 2008 excluded France, where the MON810 maize, planted on 21,147 hectares in 2007, was banned. Germany, where the GM variety was grown on 3,173 hectares in 2008, announced a moratorium in 2009. The largest area of MON810 maize (76,057 hectares) in 2009 was in Spain. The Czech Republic (6,480 hectares) was in second place followed by Portugal $(5,094$ hectares), Romania (3,344 hectares), Poland (3,000 hectares) and Slovakia (875 hectares). Romania, in the years before EU accession, cultivated largely GM (RR) soybeans on some 90-110 thousand hectares. By comparison, the acreage of soybeans has been only around 45 thousand hectares over the past two years, since the cultivation of the herbicide tolerant RR soybean is not permitted in the European Union.

\section{Economic benefits}

In 2010, the United States National Academy of Sciences (NAS) published a comprehensive study on the environmental, economic and social impacts of GM crops. The authors concluded that the application of biotechnology and the cultivation of GM plants had important environmental and economic benefits in comparison with conventional varieties used in traditional farming, even if they are not always universal, and may diminish over time.

The study concluded that biotech crops have generally less impact on the environment than conventional crops. The spread of herbicide-tolerant GM plants can facilitate the transition to conservation tillage methods, which reduce soil erosion. The spreading of Bt crops is accompanied by a decline in the use of pesticides, which benefits the environment and wildlife. The economic and agronomic consequences of the resistance developed by certain insects to $\mathrm{Bt}$ crops can be considered modest at present. However, there is no doubt that the strict tolerance levels of GMO contamination represent a serious problem for those preferring the traditional varieties, producers, processors and consumers alike.

The economic benefits resulting from the application of biotech seeds and the associated production technologies usually outweigh the additional costs. In the case of GM plants weed control is more flexible and cost-effective, and pest-related yield loss is usually lower compared to traditional varieties, thus the production cost per tonne of harvested crop is lower.

Brookes and Barfoot (PG Economics) estimated the economic gains from higher yields and lower production costs at USD 51.9 billion in the period 19962008. Pesticide use was reduced by 356 million tonnes of active ingredients, a saving of $8.4 \%$. Without biotech crops, to reach the same level of global crop production would have required the cultivation of an additional 62.6 million hectares of land during this period.

The NAS researchers underline that the impact of GM crops on the markets of conventional crops and animal products is not yet clear, and that further studies are therefore needed in this direction. In addition, they note that the social effects of agricultural biotechnology have not yet been researched carefully. 


\section{Disadvantages deriving from the delays in the approval process}

While the major grain exporting countries give a green light to the introduction and commercialisation of new GM crop varieties fairly quickly, the European Union's authorisation procedure is significantly slower and more cumbersome (months or even years longer), which has led to the prohibition, in the EU, of a number of GM varieties which are authorised for cultivation, commercialisation and use in several other countries around the world.

The adventitious presence of non-authorised GMOs in the European Union is not tolerated. In recent years, this has more and more frequently caused import stoppages, mainly of rice for human consumption, and of maize and soybeans and the derivatives of these crops (soybean meal, corn gluten feed, distillers' grain etc.) which are important feed components. Indeed, for traders it is very difficult (and expensive) to guarantee the $0.0 \%$ limit of non-authorised GMOs.

Let us take as an example soybeans, the most important and overwhelmingly imported protein source of European livestock farming. The relative weight of the European Union in the global trade of soybeans has declined steadily over the years: in the 2007/08-2009/10 marketing years, soybean imports from third countries averaged nearly 13.8 million tonnes per season, representing $16.8 \%$ of international sales, whereas a decade earlier, in the 1997/98-1999/00 marketing years, the EU-15 was the biggest buyer with a $32.8 \%$ share of global trade. By contrast, China's rapidly growing soybean imports averaged 43.1 million tonnes in the 2007/08-2009/10 marketing years already equalling 52.8\% of the international trade. Owing to the steadily growing demand in Asia, the EU market and EU requirements are becoming less important for the major exporter countries. As regards the sources of imports, there are no alternatives to the United States, Brazil, Argentina and Paraguay, since the market share of these countries is around $95 \%$

A clear illustration of the situation is that, whereas the patentees of two of the three GM soybean varieties authorised by Brazil's National Biosafety Authority (CTNBio) for cultivation in 2010 (Bayer with its herbicide-tolerant LibertyLink A5547-127 and Monsanto with its double stacked herbicide tolerant and insect-resistant Bt/RR2Y variety) will not introduce their new varieties onto the market until approval has been granted by the European Food Safety Authority (EFSA), BASF and Empraba, the joint developers of the third one (the CV 127 herbicide-tolerant variety), will not wait for EFSA approval.

The growing dominance of the GM soybean area logically impacts the market for soybeans, soybean meal and soybean oil which are non-GM or not subject to labelling in the European Union. In the case of products which are not subject to labelling, production and delivery costs are substantially higher, since more stringent requirements apply to growing, processing, storage and transport to avoid cross-contamination. Of course, exporters will reflect the additional costs in their prices. The premium for non-labelled or GMO-free produce has risen sharply in recent years: while it was only USD 5 per tonne in 2004, and USD 10 in 2005 and in 2006, since the commodity price boom in the 2007/2008 marketing year, due to a rapid contraction in the supply a USD 50-80 premium has been demanded for the high-protein by-product of soybean processing.

Feed experts agree that if the EU does not change its approval process, i.e. if the zero-tolerance remains in effect for non-authorised GMOs while these are produced in the exporting countries, livestock farmers in the Community shall on occasions (but with increasing frequency) lose their cheap protein base. As a consequence, livestock production, particularly poultry and pork, may decrease further. Today, livestock accounts for $40 \%$ of the value of the EU's agricultural production. Weakening the international competitiveness of the sector will lead to a decline in agricultural incomes, increasing unemployment and an increase in meat prices. However, in countries such as Brazil or Argentina, from where the European Union imports progressively more products manufactured from animals which have been raised on feed containing GMOs not yet authorised for use in the Community, the livestock sector may grow even faster.

While the European Commission is well aware of this threat, the authorisation procedure of GM events will not be changed in accordance with a much debated legislation proposal published in July 2010; however, the restricting or banning of biotech crops would be placed into the competence of the Member States. The Commission would draft guidelines for the development of national co-existence measures and the establishment of GMO-free areas, and would amend the existing regulations so that Member States may impose limits on or entirely prohibit all or particular GMOs in all or part of their territory if this is "public interest", without having to prove any adverse health or environmental effects. According to the United States, this autonomy of the Member States in deciding on the cultivation of GM crops conflicts with the European Union's WTO commitments.

Under another draft legislation published by the European Commission in October 2010, the lowest presence of GMOs authorised in third countries and already being in the process of authorisation in the Community would be increased to $0.1 \%$ for feed, whereas it would remain at $0.0 \%$ for food. Not only the United States, but also Canada, Argentina and Brazil have protested against this, claiming that the separation of logistics chains would impose significant additional costs on farmers, traders, food processors and feed producers equally, and that the European Union should not cause such disruptions to international trade. The majority of Member States would extend the $0.1 \%$ limit to foodstuffs too. Pragmatic experts consider that even a $0.5 \%$ limit could be accepted.

\section{What Hungary would miss...}

If there were no moratorium in Hungary, the motivation for farmers to plant biotech crops would arise from the expectation of increasing revenues and/or from certain convenience considerations. Sales revenues could increase due to a reduction in plant protection costs, lower machinery and labour expenses, and/ or the eventual improvements in terms of produce quantity and quality.

An example is corn root worm (Diabrotica virgifera virgifera), or CRW in short, which is one of the most important pests in Hungary. CRW was first 
reported on the European continent (in Serbia) in 1992. Since then, it has spread across most parts of Eastern Europe, and has maintained a permanent population in 11 countries, from Serbia to the south of Poland and from Austria to Ukraine. In Hungary, CRW was first identified in 1995 in the vicinity of Mórahalom.

Today, the imago of CRW occurs practically all over Hungary, but the most severe CRW damage occurs usually on the border of the larval spread. The extent of CRW damage is substantially influenced by weather: crop losses tend to be the greatest in seasons when June is dry, because the maize plants cannot replace the roots destroyed by the larvae.

The best protection against CRW is crop rotation; however, in areas where farmers earn the most from planting maize and therefore grow maize continuously, chemical control is quite often more common. (In the case of maize monocultures, the chemical protection of CRW-infected areas is mandatory by law.) In Hungary, soil disinfection is applied on about $15 \%$ of the area where maize is grown, whereas treated seeds are used on $14 \%$ of the planted maize area. According to the results of field trials by Gabonakutato Ltd, with soil disinfection and treated maize seeds $9-22 \%$ and 5-21\% yield increases, respectively, could be achieved in 2007-2009. The cost of soil disinfection ranges between HUF 16-21 thousand per hectare, and the extra cost of treated seeds is about HUF 9-10 thousand per hectare. With the application of these protection techniques an estimated 2.8 thousand tonnes of chemicals are released into the environment annually.

Furthermore, these methods are less efficient than the solution offered by biotechnology: according to the conservative estimates of Dillen, van Looy and Tollens of the Catholic University in Leuven, the absence of CRW resistant Bt maize in the market causes - notwithstanding the application of soil disinfection and treated seeds - a loss of EUR 15 million a year on average for maize producers in Hungary.

For Hungary, being a seed exporting country, the genetic purity of seeds is certainly important, and GM maize may pose a threat to the production of hybrid maize seeds. Although the official isolation requirements for seed production in theory prevent cross-pollination, compliance with the zero tolerance in practice is very difficult. For years, EFSA has been suggesting to the European Commission that the acceptable level of GM contamination in the case of F1 hybrid seeds be raised to $0.5 \%$.

As regards Hungarian feed imports, apart from maize (of which the country is an exporter) the situation is generally the same as in other parts of the European Union: Hungary imported 654 thousand tonnes of soybean meal and 74 thousand tonnes of other oilseed meals in 2009 , which accounted for nearly $20 \%$ of all the components used by the domestic compound feed industry. More than $90 \%$ of the imported soybean meal contains GMOs, and such feed ingredients have been used in the Hungarian livestock sector for more than a decade.

The reason for farmers not planting more soybeans in Hungary is the relatively cold weather in the north and the lack of moisture in the south during the flowering period. This cannot be compensated for by irrigation. In areas where maize yields are high (typically these are the areas best suited for soybeans too), farmers can buy more soybeans from their maize sales than they could possibly produce.

General and undue opposition to new technologies has become more or less a routine. However, in order to avoid lagging behind the competition on the international market, biotechnological research and development must be taken seriously. Plant breeding satisfies social, economic and market demands and interests. Society demands its food needs to be met in compliance with certain quality requirements changing in space and time, and with less negative impact on the environment. To increase and stabilise yields is an economic interest and, ultimately, the market is looking for competitive products. With additional (and reasonable) expenses, coexistence can be realised, and the problems in plant breeding, seed and commodity production, processing, trade and consumption arising from the unwanted biological and physical transfers of genes can be prevented by adequate regulations.

\section{Summary}

Biotech crops can deliver substantial environmental and economic benefits compared to traditional plant varieties. The importance of the EU market and EU requirements for the major soybean and maize exporter countries is declining over time. In Hungary, if $\mathrm{Bt}$ maize were allowed to be grown, maize producers could gain EUR 15 million surplus a year.

Note added to the proof: During the printing process of this book, ISAAA published the latest data on the GM crop cultivation in 2010 Worldwide, which exceeded 148 million hectares (www.isaaa.org). 


\section{GMOs and environmental safety}

\section{ERVIN BALÁZS, LÁSZLÓ SÁGI}

A frequent remark on the environmental release of genetically modified (GM) living organisms is that even scientists have different opinions on the question. Beside the inherently democratic nature of science, this is due to the fact that experts studying ecosystems are reluctant to accept a human intervention that changes or may ruin natural habitats in any way, no matter if it is a highway, a residential community area, a football field, a factory, a shopping centreor agricultural activity. Their efforts to protect habitats are noble and must be appreciated, which should be respected by any responsible person. However, the conflict between the interests of a farmer and an ecologist is obvious, considering that agronomists alter natural habitats by removing all the plants and replacing them by only one or a few plant species, which in turn have to be protected by various mechanical, chemical or biological means from plants, pathogens and pests of natural ecosystems being harmful to the cultivated crops. This conflict cannot be resolved unless both parties are committed to come to a compromise or even an agreement. Independently of the rate of increase in the population of the world in the last thousands of years and particularly the last century, in our age it would be highly obsolete anyway to gather our food like prehistoric people.

The increase in agricultural productivity has made it possible to supply the world population with food of suitable quantity and quality, notwithstanding that millions still die of malnutrition or starvation even these days. During the thousands of years of its history, agricultural production has generally been performed in harmony with nature, although it occurred quite frequently that certain trends were needed to be subsequently modified in the light of experiences. The progress in chemical industry and the intensive applications in agriculture leading to excessive use of chemicals as well as the resulting harmful environmental effects called for the reconsideration of plant protection and nutrient supply issues. The book 'Silent Spring' by Rachel Carson that received considerable publicity in the 1960's brought to the attention of society that an agricultural production scheme based on biological plant protection and ecological approach has to be developed instead of employing extreme chemicalisation. Now that we entered the century of biosciences, and the achievements of molecular biology are applied by modern plant breeding, the agricultural utilization of plants furnished with new traits using gene technology methods is protested against by opponents referring exactly to adverse environmental effects attributable to chemicalisation. With regard to the major views expressed in opposing opinions, the following are worth to be considered.
Although GM plants have been cultivated for over one and a half decades and on an ever-increasing area (as much as 148 million hectares in 2010), we basically still do not have enough experience gathered over a sufficient period to ensure reliability.

The opponents should specify what length of time they consider as sufficient and why: ten years, fifty years, a hundred years or even more? The answer depends, of course, on the given species, since bacteria divide every 20 minutes, thale cress (Arabidopsis) that has a short life cycle sets seeds in 6 weeks, whereas other plant species have only one generation a year. How many generations should be taken into account? - one might ask. In accordance with the plant breeders' experience of several decades, excellent plant varieties are usually in cultivation for 7-10 years, whereas this period is, for obvious reasons, significantly longer for woody plants. This also implies that while the dispute on the length of period regarded as sufficient has not been settled yet, the first generation of GM plants are already being gradually replaced by new and better GM varieties.

On the other hand, it has to be remembered that over $95 \%$ of the plant species that currently determine Europe's agriculture originates from other continents, considerably changing the landscape of vegetation in the course of time, which was facilitated by the great exploring expeditions and above all by the discovery of America. The plants of the New World including bean (Mexico, Guatemala), potato (Peru, Chile), tomato (Peru and Mexico), chili pepper (Bolivia and Mexico), maize (Mexico), just to mention the most important ones, joined just a few centuries ago the group comprising stone fruit species of Asian origin, wheat brought from Asia Minor and cowpea originally from Africa. So the question could be raised: Actually, how long is it since we have 'reliable' knowledge in connection with our field crops with regard to cultivation and environmental effects? Analysing the issue of time paradigm, in consideration of these centuries, which can be regarded as a single moment in terms of geohistory, a Hungarian example can also be mentioned: red pepper, which is now claimed to be a Hungarian speciality, began to spread in Europe only about 200 years ago during the Napoleonic wars, when the black pepper shipments from India got stuck due to the blockade of European harbours, and black pepper could be substituted through the production of hot red pepper. In Hungary, it was only in the late $19^{\text {th }}$ century that red pepper was brought into cultivation, partly due to the immigration of market gardeners and partly by the crop's quick adaptation to the excellent climatic and environmental conditions of the Carpathian basin and by the establishment of a secondary gene centre here. Another example is the cultivation of kiwifruit in Hungary dating back to a few decades. Has anybody ever complained about the introduction of the gene pool of this species, several thousand genes completely alien to the country's flora, not to mention the symbionts, pathogens and pests arriving together with the plant, whereas fierce anger is triggered if a plant species cultivated for centuries is furnished by a few exogenous genes with traits that are more favourable from the aspect of production technology? Well, no one has ever protested, and kiwifruit is grown 
without any problems - just as all the other crops that were introduced into Europe without any knowledge in food safety and ecology.

The maintenance of biodiversity is in danger by the cultivation of GM plants, say the opponents of modern biotechnology.

As was stated also in the introduction, it is an incontrovertible fact that the alteration or destruction of any natural habitat is accompanied by a significant decrease in or loss of the biological diversity of the given area, irrespective of whether GM plants or traditional varieties are grown or organic farming is carried out in that area. The species richness of a natural habitat greatly exceeds that of agroecosystems, under any circumstances. This is attributable to the fact that the life of that habitat is disturbed by various human interventions, regardless of whether it is a mechanical intervention (ploughing, soil loosening, etc.) or chemical treatment (supplying nutrients, weed control, plant protection). Here, we would like to refer to a comprehensive experiment of several years implemented in major potato production regions in Germany, studying the changes in the soil ecosystem in terms of both the number of species and the number of individuals per species during the cultivation of various potato varieties and a GM variant of one of these varieties. These thorough experiments led to the conclusion that the difference between the biodiversities of soil ecosystems was greater among the different varieties than between the GM and non-GM variant of the same variety: in the case of the latter two, no statistically significant difference was found, unlike between the soils of different varieties. Experiments conducted with non-GM plants revealed that crop rotation caused considerable variation in the microbe composition and species richness of soil. Climatic effects such as drought, frost and inland water also lead to substantial fluctuation in soil ecosystems. Thus, the production of GM plants by no means causes such great and irreversible changes in the biological diversity of agricultural and neighbouring environment as it is claimed and desired by the opponents, especially when compared to pesticides and intensive tillage. In order to maintain biodiversity, it is in fact the most reasonable conception to produce food in the ever-decreasing arable lands by technologies as effective as possible or (save the mark) through the cultivation of GM plants, by which certain habitats, for instance those less suitable for farming could even be withdrawn from cultivation and given back to nature. GM plants may thereby indirectly contribute to the improvement of biological diversity.

The accusation of reducing biodiversity may also be interpreted to mean that successful GM plants would narrow the range and genetic background of cultivated varieties. This process has in fact continued since the beginning of traditional plant breeding via the gradual disappearance of landrace cultivars up to the present days. In the United Kingdom as many as 10 bird species suitable for breeding have disappeared, perhaps forever, in the last 20 years. In contrast, if the increasing number of GM plant varieties cultivated worldwide is considered, including those that have already undergone the procedure of authorization and are only awaiting approval, one can in no way conclude that GM plants reduce the choice of varieties. Finally, it is worth emphasizing again that most of the present GM products are only the modified variants of successful cultivars developed earlier, therefore they do not increase or reduce the genetic variability of the complete range of varieties at all, but are indifferent in this respect.

It is a repeated accusation that if a GM organism is released, it cannot be withdrawn from nature any more.

No reasonable assumptions are contained in this concern either, as varieties are routinely withdrawn from field production year by year. This claim can be refuted simply by referring to a less glorious chapter in the history of Hungarian agriculture, when the production of cotton plants, started in the 1950's under political pressure failed within a few years. Since that time, no cotton plants can be found in the flora of the country, and neither has the survival of its genes been reported in spite of the fact that it was grown on several thousands of hectares 50 years ago.

At this point, it is worth noting that field crops may actually assist the preservation of wild variants, a good example of which is the conservation of teosinte, one of the ancestors of maize, in a practically unchanged form. Teosinte is a common weed in some parts of Mexico, whereas it is still grown as a fodder crop at high altitudes on some hillsides. Maize is able to pollinate teosinte without substantially modifying its genome (something that couldn't be done anyway, since the difference between maize and teosinte e.g. in the genes responsible for ear morphology is only 5 genes), thereby ensuring the survival of this ancient species on the verge of extinction.

A related objection is that the introduced GM trait is transferred to the natural ecosystems, leading to unfavourable outcomes.

First, it must be clearly understood that the pollen's ability to fertilize a related species is not modified by GM techniques, at least not by those applied so far. As this is a natural ability of plants, any other (non-GM) feature of cultivated crops such as herbicide resistance developed via induced or spontaneous mutations may in the same way be transferred into natural habitats. In addition, the ways of reproduction in higher plants also has to be considered: there are windpollinated, insect-pollinated and self-pollinating species. In the latter case, fertilization takes place in a closed system, hence the chance for a GM trait to escape or enter is negligible or zero. Regarding wind-pollinated plants, both the time of flowering and the viability of pollen have to be taken into account. For example, the viability of the pollen grains of grasses and cereals in the wild is highly limited and rarely exceeds 2 hours. What's more, gene transfer via pollen is restricted by several well-defined mechanisms in nature. It also has to be emphasized that plants are only able to fertilize their own species and a few other related species; consequently, if a crop species has no wild relatives in a 
particular continent or region, biodiversity is not at risk at all. Moreover, as mentioned above in the case of maize and teosinte, cultivated crops might even have positive effects on their ancestors.

The case of rice is different, because a major restricting factor of its efficient production is the spreading of wild rice, a weed. Wild rice, which at present can only be controlled by herbicides, is freely pollinated by cultivated rice; therefore, if herbicide resistant GM rice is grown, this trait is likely to be transferred into wild rice, thus reducing the efficacy of weed control. The extent of the transfer is influenced by many factors. On the basis of observations accumulated in several centuries, traits from cultivated crops are transferred into related wild species at a very limited rate due to the simple reason that they either flower at different times, or - in case fertilization does occur - natural defence mechanisms prevent the exchange of traits, or the progenies generated this way might be infertile or might have reduced viability causing early decay. Thus, traits transferred into wild species are not maintained in large quantities and for a long time in nature.

New virus variants harmful to nature can be created in virus-resistant GM plants, the opponents claim.

Virus resistance in GM plants can be developed by the introduction of a nucleic acid sequence derived from the genome of a given virus, for instance by the application of the gene encoding the viral coat protein. Viruses do employ gene exchange, recombination between virus strains during natural evolution. Even though the locations of recombination, the so-called hotspots in virus genomes are known, no new virus variants have been found in GM plants in the past nearly 20 years. This has several reasons: on the one hand, the copies of the inserted viral nucleic acid sequence are located elsewhere (in the nucleus) than the location where viruses multiply (the cytoplasm). Accordingly, the two nucleic acid fragments assumed to recombine are physically separated from each other. On the other hand, new virus strains can only be generated during the evolution of viruses if the virus is able to multiply efficiently, which requires susceptible plants. But the level of viral multiplication in resistant GM plants is so low (if any) that it is hardly detectable even with the highly sensitive present-day molecular methods, as demonstrated in our investigations performed in the frame of an international co-operation (Dietrich et al, 2007, Environmental Biosafety Research, 6: 207). In accordance with the above, it is in fact the virus-resistant GM plants where the conditions of recombination required for the creation of new virus variants are not available. At the same time, this also means that the development of new virus variants through recombination between different virus strains is indeed more likely in non-GM plants.

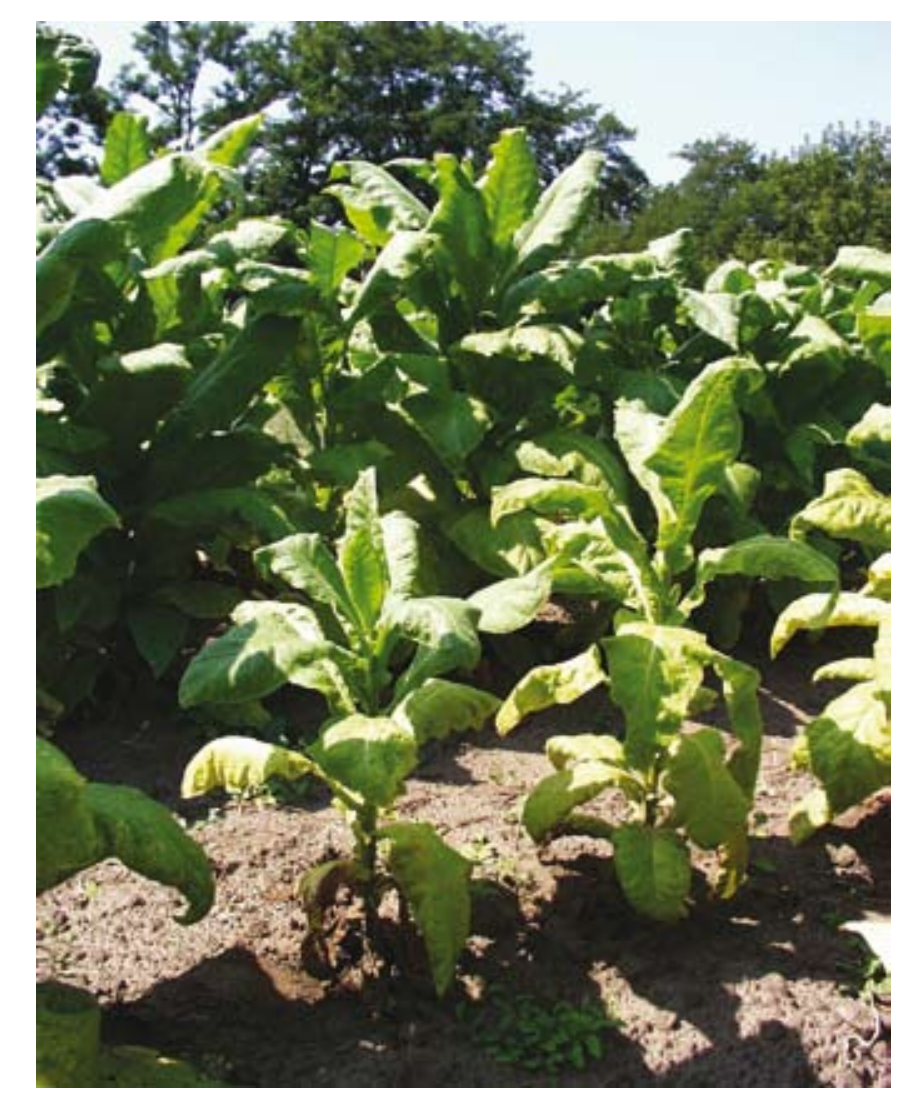

Figure 15.1. Transgenic virus resistant tobaccos (back row), in the front row infected non-transformed parental lines. (Photo taken by Ervin Balázs)

By the spreading of herbicide-resistant GM plants, the use of herbicides has not been decreasing but is in fact increasing, and resistant weeds appear in ever-increasing numbers, and what is more, the company holding the licence for a herbicide controls the market, the opponents presume.

This issue requires detailed consideration, because, on the one hand, the chemical load of arable lands has been decreased significantly by the application of modern herbicides, which are used in very small (micro) amounts, as compared to traditional herbicides. On the other hand, by growing herbicide-resistant GM plants on a rapidly increasing area, the total amount of herbicides used has indeed been increasing. However, in this case the crucial aspect is that the overall herbicide use is significantly lower in countries growing tolerant GM plants (for instance the annual reduction in Canada is 
6000 tons, which exceeds the total annual use in Hungary), because obsolete herbicides applied in large quantities could be replaced by others that are more environmentally friendly and more efficient. A good example is glyphosate, which was registered 35 years ago and has been on the market ever since. Its popularity can be attributed to its favourable characteristics: it is a nonselective herbicide with a broad spectrum and is translocated in the plant in every direction (systemically). Its active agent is practically non-toxic to mammals, birds, insects and to the majority of bacteria. It does not accumulate in animal tissues and is proved to be non-carcinogenic. Glyphosate does not have long-term effects in the soil, as the active agent is strongly bound to soil particles, preventing it to be washed into groundwater. For this reason, it does not damage the field crops emerging after the treatment

Glyphosate is decomposed by soil microorganisms to its components, which are natural compounds (ammonia, phosphate, carbon-dioxide and glyoxalates). The average half-life (the period of time it takes for the amount of a substance undergoing decay to decrease by half) of the active ingredient of glyphosate in the soil is 45 days. Due to the relatively rapid decomposition, it does not accumulate in the soil, and $90 \%$ of the active ingredient will be transformed into natural substances within 6 months. Bacteria performing this biotransformation are not damaged by the agent; on the contrary, they are supplied with phosphorous. The media became interested in glyphosate use only after the first glyphosate-resistant GM plants had been created; through their involvement in field cultivation, glyphosate became a part of professional agriculture, particularly in the case of the most important crops with high economic value such as soybean, maize and canola. It is no mere coincidence that rapeseed production in Canada is based almost exclusively on herbicide-resistant GM plants: the reason is their environmentally friendly nature, not to mention their economic profitability.

As a result of the widespread application of glyphosate - just as it could be observed with numerous other pesticides - weeds resistant to the agent appear after a while, which must be controlled using alternative chemicals, in accordance with the general practice of plant protection. This course of events is not restricted to GM plants; it is also unavoidable in the case of non-GM crops. This is exactly why new GM plants resistant to two different herbicides have been developed, enabling the alternate application of the most widely used chemicals and significantly reducing the chance of the development of (double) resistance.

Conservation tillage, the interest in which increased worldwide owing to the energy crisis in the 1990's, became feasible due to the growing of herbicideresistant GM plants. The technology has spread mainly in countries with advanced technical background. It is explained by the fact that conservation tillage requires machinery that is different from traditional machines and that the investment is returned earlier if farming is carried out on larger area. The advantages of minimum tillage include the prevention of soil erosion attributable to extreme weather conditions, the reduction of weather-dependence in plant production, the preservation of favourable soil structure and the protection of organisms living in the soil. The reduction in the number of tillage operations and in diesel oil usage also results in a significant decrease in carbon-dioxide emission and greenhouse effect.

Other objections against the increased use of GM plants resistant to a herbicide, in this case glyphosate originate in the fact that the proprietary rights of glyphosate are owned by a multinational company, which means a monopoly situation in the market; however, after the expiry of the patent in 2000 this concern is not so steady any more.

Each cell of insect-resistant GM plants produces a bacterial toxin that is harmful to the environment, to the animals consuming these plants and even human beings, sounds the criticism.

Let us start with the facts. The crystal protein produced and stored in the soil-born bacterium, Bacillus thüringiensis $(\mathrm{Bt})$ is called $\mathrm{Bt}$ toxin by researchers, because it is harmful to certain insect families, or can even be lethal to some insect species. The method of biological plant protection using the bacterium itself or the toxin preparation derived therefrom is not disapproved but in fact supported by the opponents of gene technology. However, in the event the substance toxic to insects is produced by the plant cell, it is immediately declared as poison. This can be called nothing else but double standards. Apart from the above, there are two basic facts that have to be known regardless of whether it is about biological plant protection or GM plants. Bt toxin is specific to certain insect families and is not toxic to other insects or living organisms, because they do not have the appropriate receptor on the surface of endothelial cells to which the toxic protein has to bind in order to exert its effect. As humans lack the appropriate receptors in their guts, they cannot be affected by Bt toxin at all. In such cases, the toxin can be considered as a common harmless protein, which is simply decomposed in these organisms, for instance in the gastric acid of humans. In nature, various Bt proteins are known, the genes of which have been introduced into several plants of economic importance such as maize. The temporary and limited moratorium announced in Hungary on the GM maize resistant to the European corn borer (MON810) is of symbolic value, because substantial corn borer infestation occurs only in some years in Hungary and can in fact be prevented by chemical plant protection. At the same time, it has to be mentioned that the consequence of corn borer infestation is more remarkable than the infestation itself: the fungus Fusarium can more easily enter the plant tissues through the lesions caused by the chewing of corn borer, and the mycotoxins produced by these fungi lead to enormous economic losses through the contamination of forages, since part of the forage produced becomes useless (those who feed animals with such forage only enhance damages). The case is different with another Bt protein, which would/could provide protection against a pest causing by far greater losses, i.e. corn rootworm, resulting in inestimable economic and environmental benefits. It is a well-known fact that by growing GM plants producing Bt toxin, significant savings in chemicals (and costs!) can be 
achieved worldwide due to the omission of chemical sprayings. One expressive piece of information: the yield of Bt cotton plants in China increased by $10 \%$ in 2009 (we are speaking here about 'only' 7 million farmers!), while the amount of pesticides used decreased by $60 \%$.

In connection with the application of Bt toxin for plant protection, it is reasonable to raise the question of resistance developing in insects. Resistant individuals and populations can be selected over time for almost any of the plant protection chemicals, as demonstrated under laboratory circumstances. The resistance of insects to Bt toxin is delayed or prevented by growing insectresistant GM plants and susceptible plants side by side, creating refuges where insect populations susceptible to Bt toxin can survive. This can be done by using the seed of a susceptible plant variety at a ratio of about 20 percent in the area, or by sowing rows of non-GM varieties at the edges of the fields. Similarly, the application of crop rotation also hinders the development of resistant insect populations, as it is now done in the frame of traditional plant protection technologies, since the development of Bt-resistant insect populations is attributable to the Bt toxin used as protective agent and not to GM plants. Thus, the case is the same with Bt-preparations applied in organic farming.

Another frequently cited concern is the effect of GM plants producing Bt toxin on non-targeted living organisms, in this case other insects present in the agroecosystems and having important roles (such as ladybirds, lacewinged flies, etc.). Here the toxins produced by the plant have to be considered, as each toxin only affects insects that are closely related to the pest. For instance, the Bt toxin in MON810 GM maize (banned in Hungary) has no effect either on $10 \%$ of the 60 species examined from the order of Lepidoptera or on any other insects. In the case of the European corn borer, it is obviously the butterflies and moths in the maize fields that are at risk, but not all of them. The peacock butterfly, a protected species in the Pannon ecoregion feeding on common nettle, a weed found in maize plantations and their environs, is mentioned from time to time. In the event the pollen from a maize plant producing Bt toxin gets onto the leaves of nettle in large quantities, the caterpillar of the peacock butterfly consuming such leaves may indeed be killed. This was shown under artificial laboratory circumstances; in the fields, however, it is very rare that pollen in sufficient amounts to harm the caterpillars (at least 300 per square centimetre) accumulates and is retained on nettle leaves. In actual fact, the average pollen number per square centimetre is below 100, which can be reduced a hundred-fold by wind, but even a single precipitation event of $10 \mathrm{~mm}$ decreases it by $70-80 \%$ as was demonstrated in field trials. Let us finally clarify this issue by pointing out that agricultural and household insecticides and even the Bt preparations applied in organic farming are far more harmful to protected butterfly and moth species and to a broader range of beneficial insects than GM pollen. Insecticides may totally kill insects in a given field, but nature is able to recover even from such destruction: after some time new individuals simply migrate there. Likewise, climatic and frequent weather changes pose a great threat to insects and insect populations with vast numbers of individuals killed - without being noticed. Not to mention that millions of beneficial and protected insects and migrating butterflies and moths end up killed on windscreens and engine hoods of cars speeding along the roads (Figure 15.2.). Why is it that environmentalists worrying about butterflies and moths only protest against GM plants (unjustifiably, as we see), when they could do it with good reason against e.g. the automotive industry expanding worldwide?

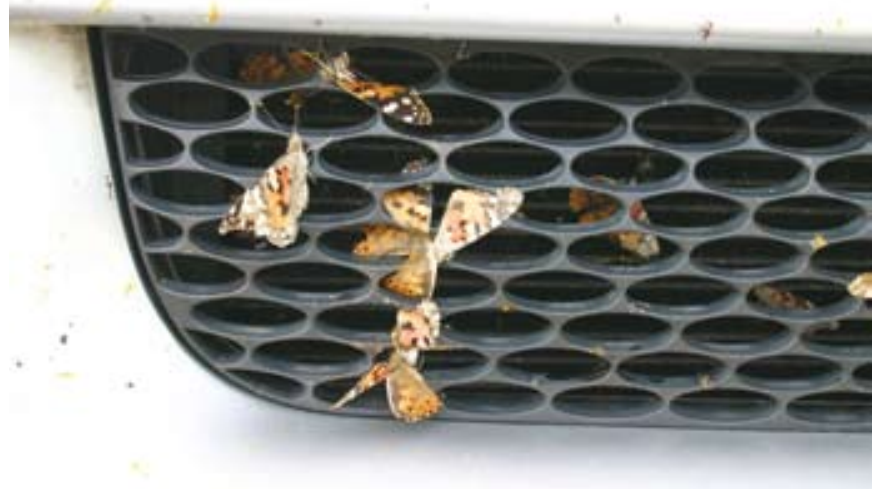

Figure 15.2. Smashed painted ladies on cars front (Photo taken by Ervin Balázs)

Another repeated argument is the effect of toxin protein remaining and slowly decomposing in plant parts in the stubble after the harvesting of $\mathrm{Bt}$ toxin-producing GM plants on organisms living in the soil. Here it has to be stressed again that the Bt toxin may only exert its biological and, in certain cases, toxic effect on organisms that are in the same insect family as the target pest. However, this should not be over-emphasized, as pest-related species are uncommon in the soil. The Bt protein may remain in the soil extendedly, since it binds to clay minerals, which reduces the speed of its decomposition, but - for the exact same reason of being bound - it will be biologically inactive, hence non-toxic. In contrast, the Bt protein will decompose much faster in sandy soils. No reliable experimental data (obtained in trials performed under real-life circumstances and in adequate number of repetitions using proper controls) has been presented up till now that could demonstrate any factual negative effects of Bt protein residue on soil biology.

Finally, the risk that the introduced genes are transferred into other living organisms is a common concern, with special regard to soildwelling microbes and the phenomenon of horizontal gene transfer.

The process of gene transfer between microbes has become a well-described phenomenon with the expansion of scientific knowledge. Since it is an integral 
part of evolution, once a GM microbe is released to the environment, the transgene may be transferred into other microbes. However, the shifting of a gene from GM plants into microorganisms is restricted for several reasons, for instance due to the lack of competence (receptivity) essential for DNA uptake by bacteria. The subject of antibiotic resistance genes is not explicated, because they are not used in the course of genetic modification any more, or are removed from GM plants. Here we only refer to the fact that for the generation of GM plants only those antibiotic resistance genes were employed for which the corresponding antibiotic agent had been withdrawn almost completely from veterinary and human therapy, therefore the pharmaceutical application of antibiotics was not at risk at all. It is a well-known and unfortunate fact that antibiotic resistance quickly develops in nature, causing real problems in human health care these days, but - to emphasize again - this cannot be associated with antibiotics used in plant biotechnology. By the way, it is also known that about 50 percent of microbes in human intestinal flora are resistant to some kind of antibiotics. Accordingly, the negligible chance that GM plants might modify the antibiotic resistance pattern of intestinal microbe populations is insignificant in comparison with the biological importance of GM plants. It is worth noting that, as revealed by a French study, over 50 percent of microbes in soils that had never been cultivated (prairies) had some sort of antibiotic resistance, whereas the same value in regularly cultivated soils only slightly exceeded 2 percent.

There are of course more arguments that the opponents of GM-technology might raise; here we only commented on the most common general concerns, revealing their inadequacy and the fact that they hardly ever contain justifiable reasoning that would prove the negative environmental effects of GM plants.

Almost each of the objections in connection with environmental effects calls attention to possibilities that cannot be excluded and have therefore to be correctly studied, in spite of the fact that they are very unlikely to occur and their effect is therefore insignificant. What is more important is that alternative gene technology solutions pose much less threat than do industrial farming technologies; moreover, it can be stated that they are much safer from the aspect of animal and human health

\section{Recommended reading}

Park, J. L., McFarlane, I., Phipps, R. H., Ceddia, G. (2011): The role of transgenic crops in sustainable development. Plant Biotechnology Journal, 9: 2-21.

\section{The process of gene technology regulation}

ERVIN BALÁZS

In Central Eastern Europe, the legislative regulation of gene technology was first developed in Hungary in 1998 to enable the practical utilisation of the results of biotechnological research. The Act on Gene Technology Activity is in conformity with the corresponding law of the European Union and its strict rules do not justify the maintenance of the Hungarian Moratorium.

The necessity of the regulation of gene technology was pointed out by Hungarian top scientists at the very beginning: Professor János Szentágothai, who was the President of the Hungarian Academy of Sciences at the time, initiated a statement in this issue to be made by the scientific body in the mid 1970s, at the same time as the American biologists declared their opinion in 1975 in a document known as the "Letter of Asilomar". In the period when biological weapons were banned, the American scientists who had worked out the basics of genetic engineering referred to their moral responsibility and asked their government to regulate the field of gene technology even at the sacrifice of suspending their experiments until such regulation is elaborated. The government of the United States of America, recognising the results and exceptional potential in molecular biology for the future of mankind, urgently started the process of legislation, the result of which was the NIH Guidelines for Research Involving Recombinant DNA Molecules issued by the American National Institutes of Health in 1975. These Guidelines were very strict at the beginning, but due to their regular and controlled revision the experiments which previously could only be carried out under limiting conditions can now be performed under simpler circumstances. The American example was followed by the OECD, the organization of economically developed countries, which published its recommendations on the safety considerations of recombinant DNA in 1986. These suggestions serve as guidelines for the regulating activity of the individual countries and define the frames that are recommended to be taken into consideration. These considerations were taken as a basis by the European Union as well when issuing in 1990 the directives on the contained use of genetically modified microorganisms (EU Directive No. 219/1990) and on the deliberate release of genetically modified organisms into the environment (EU Directive No. 220/1990), which are still determining.

In consideration of the importance of the subject, almost each of the international organizations, including the World Health Organization (WHO), the Food and Agriculture Organization (FAO), and of course the United Nations Environment Programme (UNEP) has set down its recommendations for its own special field. The document published by the latter, the International Technical Guidelines for Safety in Biotechnology (1995) is the one that focuses principally on the possible environmental effects. Later the countries that wished to develop or use the technology because of its advantages in terms of economy, 
environmental protection and human health enthusiastically started one after another to make national rules, decrees and even acts.

The changes in the political regimes in the Central and Eastern European countries in the 1990s lead, in the hope of accession to the European Union, to the effort to be in conformity with the rules in force in the then member states concerning the regulation of the application of genetic engineering. This was manifested in a regional activity, which was supported by the experts from the Netherlands, Germany, the United Kingdom and Austria as well, aimed at establishing controlled conditions for the application of gene technology in our region with as little delay as possible. Scientific conferences were organized annually with the participation of the specialists of the region in order to develop the required regulations and significant professional assistance was provided to ensure that this economic region catches up with other countries of the European Union.

Hungary did pioneering work in this field not only by being the host and the organizer of these regional meetings (1995 Keszthely, 1996 Budapest, 1997 Smolenice, 1998 Bled, 1999 Pruhonice), but also by being the first country in the region to prepare and issue a governmental decree on the regulation of the activity of genetic engineering, which was accepted by the Parliament in March 1998. We are very proud to mention that the experts of both the Slovak Republic and Slovenia took the text of the Hungarian governmental decree into account when developing the regulations of their countries. The framework law of Hungary also used the approach that the regulation should be developed in accordance with the developmental process instead of the new features of the end product, just as it is regulated in the European Union. The substantial merits of the Act included the provision of complete transparency, the publication of the authorization system of genetic engineering activity and the establishment of the Gene Technology Committee. The members of the Committee at the time were assigned by the Hungarian Academy of Sciences, the competent ministries and a large number of social or civilian organizations. Interestingly enough, the representatives of the users were left out with reference to interestedness; however, the representatives of certain civilian movements who in fact had adverse interest in the application of gene technology were involved. Later, the executive instruction was temporarily modified and the bio-industry could also represent itself in the Committee. This situation soon ended, when the Hungarian rules that are considered to have been essentially good were started to be rewritten at the time of the accession to the European Union, in view of the legislative revision initiated in the Union and of the harmonization of laws. The process was further complicated by the demand for the regulation of coexistence (the production of GM and non-GM plants side by side), the concept of which arose meanwhile. In Hungary just like in the international scene, the experts were pushed into the background in this process and their roles were taken over by the representatives of voluntary green movements. The management of the Hungarian Gene Technology Committee also changed in this manner, as a consequence of which the continuation of field experiments that had been authorized at the beginning and the acceptance of new applications failed in large numbers. On the basis of the Committee's recommendations, the authorities specified requirements for the experiments that were not justified professionally and could not even be interpreted, proving that the members of the Committee were not aware of the basics of plant breeding. To make things even more tangled, the orders issued to control coexistence were scientifically unfounded, such as the unreasonable increase in the genetic isolation distances that eliminate the possibility of an open field release. Another similar obstacle is that the approvals of all the owners and growers of the neighbouring fields are required for the production of GM plants. Due to the confusion in the possession of land in Hungary, such experiments - and not to mention field production are made impossible by this rule. It cannot be concealed that a Member of the Parliament said at the time of the amendment of the Act that even though they did not oppose GM technology, they would specify conditions that make its application impossible. Unfortunately, that is exactly what happened. The number of applications for and implementations of open field trials has been reduced to a minimum and Hungarian researchers set up their experiments in nearby countries. The political changes that occurred in the last few months and restructured the governmental organization have put the regulation of gene technology into an "ex lex" state, since the ministries assigning the members of the Gene Technology Committee have ceased to exist, and thus certain delegates have become illegitimate. Moreover, the international opinion on Hungarian science has turned extremely negative because of the fact that our country - giving in to green political pressure - announced a moratorium on the production of a genetically modified maize (MON810) and even though its importance is not really significant in Hungarian production, the politicalsocial influence is all the more important as it endangers the social support of the widespread usage of an economically profitable GM plant in the future. It is also reasonable to ask why Hungarian farmers are prohibited from this economically competitive technology when GM products, in compliance with commercial treaties, are allowed to be imported for foraging.

In terms of nature protection, the adoption of the Protocol on Biosafety in the year 2000 was a very considerable event. The demand reasonably expressed in the frame of the Convention on Biological Diversity (CBD) is that the spreading/ distribution of GM organisms among countries, regions and continents has to be regulated in order to protect biological diversity. This regulation known as the Cartagena Protocol is, by the way, the only legally accepted international document in the subject that was ratified by Hungary (2004). It must be noted that this regulation was also initiated by the scientific community aware of their responsibility, and they participated in its formulation at the beginning of the negotiations. Regrettably, scientists were increasingly relegated to the background, meanwhile the participation of legislators and politicians increased and the influence of civilian movements gained ground (not concealing the positive sides of it). Today, the CBD expert list (to be found at http://www.cbd. int/countries/?country=hu) includes almost nobody from the scientists who 
initiated and participated in the elaboration of the Cartagena Protocol. This situation basically determines the social criticism on the professional complaints in connection with the implementation of the Cartagena Protocol. The convention was adopted in the spirit of the concept of precautionary approach; however, it was later arbitrarily altered in the legislation of the Union to the precautionary principle, which - also because of significant differences in interpretation - induces opposing attitudes in the New World and in Europe. Not underestimating the need for regulation, it can be established that the decrees of some countries and especially those of the European Union are so bureaucratic and over-regulated that, as a result, the authorization procedure of a GM plant in the Union may take as many as 3 years, whereas the same process in the United States of America or Canada takes not more than 6-8 months. The cost of a procedure in the States is about 5 million dollars, whereas in the Union it costs roughly 8 million euros. Because of these costs university research units or research institutes are unable to apply for the authorization of GM plants, making way for well-capitalized large multinational companies. The application of genetic engineering technology is thus limited mainly to hybrid plants that offer significant economic benefit and can be protected under patents.

The importance of food safety has been recognized by the European Union, with special regard to the fact that considerable amounts of products and raw materials are imported for food industry use from other continents, where chemicals already banned in the countries of the Union are still used in agricultural production. Furthermore, these goods may be contaminated with microorganisms that pose a threat both to the health of European people and to the environment. To eliminate the risks, the European Food Safety Authority was established in 2002 with a scope of activity that includes all issues related to food safety. Its operation and professional competence can be considered as equivalent to the corresponding governmental bodies of the United States of America, Canada and Australia. One of the important tasks of this authority is to develop a professional position in relation to GM organisms. Regrettably and in spite of that the body was organized in the Union as a result of a political decision, the politicians of the Union are sceptical and they often accuse the expert positions of being biased, whereas the overseas societies not only trust the professional standpoints of the government but also accept and support them. The professional activity of the organization of economically developed countries, the OECD may help a lot in promoting the acceptance and understanding gene technology, since they assist the regulation of genetic engineering in the individual countries by preparing so-called consensus documents (http://www.oecd.org/ document/60/0,3746,en 2649_34387_46720508_1_1_1_1,00.html).

During the commercial production of GM plants, which has now been practiced for more than 15 years without any negative effects on the environment or on animal and human health, and which is now performed on over 137 million hectares, a considerable amount of knowledge has been gathered that would justify the simplification of strict rules, and in certain cases the regulation could be reduced to the liability of notification. In the United States there are some indications for this trend, as shown by the deregulation of virus resistant GM plants. However, the most important notion for the future is that regulations should not be made for the sake of regulating. 


\section{GM plants - the media - the public}

\section{JÚLIA GIMES}

According to the results of the latest survey based on representative samples from 32 European countries (Europeans and Biotechnology in 2010, Winds of change? Publications Office of the European Union, Luxembourg, 2010, ISBN 978-92-79-16878-9 doi 10.2777/23393; http://ec.europa.eu/public_opinion/ archives /ebs/ebs_341_winds_en.pdf), the majority - 53 per cent - of Europeans are optimistic about biotechnology and gene technology, whereas 20 per cent are unable to form an opinion. On the other hand, genetically modified (GM) agricultural products have much less public support: opponents outnumber supporters in each country by two or three to one and even this not-so-vigorous support shows a declining trend. In addition to the fact that safety is paramount for Europeans, they do not see that the application of gene technology techniques would have any benefit in agriculture. While gene therapy (which involves the modification of the human genome and where healing is the aim and, if everything turns out well, the result as well) is approved by 63 per cent and rejected by only 37 per cent of the respondents, these rates are just about the opposite in the case of GM food.

So far GM plants have been produced in six European countries (Spain, Portugal, the Czech Republic, Slovakia, Romania and Poland) on a total area of around 95 thousand hectares, whereas their production is banned in six countries (France, Germany, Greece, Luxemburg, Austria and Hungary). The opinion on GM food in Hungary is similar to or slightly more positive than the European average, since it is approved by 32 per cent and rejected by 68 per cent of the respondents (the rate in Europe is 27:73). In the countries where production is banned, the level of approval is also low (10-23\%) among the inhabitants, lower than the European average, with the exception of Hungary (32\%).

The survey also reveals that European citizens are quite familiar with the topic of GM food. Approximately 50 per cent of the respondents not only have heard of these technologies but they had also engaged in active discussion or information search on the subject. Only 18 per cent is completely unaware of GM food. This level of familiarity is obviously attributable to considerable media attention which is typical in Hungary as well.

The intensive interest is well characterised by the almost 30 thousand search results published in the last year in Hungarian language or from the location of Hungary are given by an internet search engine for searches when the acronym GMO is combined with the Hungarian word for food, plant or maize. There are at least 50-100 appearances on the subject in the national media, the majority of which are - in conformity with the public opinion and the official position supported by all of the political parties - negative.

Those working in the information services sector are in a fairly difficult situation if they want to discuss this delicate topic influenced by diverse interests and viewpoints. A part of the problems is connected to the condition/state of the media:
The acceleration of information flow and keen competition do not facilitate the thorough exploration of any subject that requires a lot of time and effort. The evaluation of the results of gene technology is primarily a scientific issue. The identification of the expected consequences and risks of the practical utilization of these results would also be a scientific issue, although it belongs to another specific field. However, scientific results are sometimes hard to understand not only for the readers of news but also for the 'producers' thereof. It takes much time to adapt scientific results to a form that is comprehensible for the public and is still of the required standard, but much time is rarely available. Threeday-old news is considered outdated; even the news about scientific discoveries goes round the world within a day, and for the next day another one is needed. Under such circumstances, it is not surprising that the published information is often unfounded, false and misleading. It requires much more endeavour to work from the original source and much more time to gather information and consult with the experts of the subject. Besides forced haste, the pressure to be catchy enough is another outcome of sharp competition, which also reduces the quality of information. Scientific findings rarely hit the headlines. Science usually proceeds at a slow pace through lengthy and tiresome work, the reports of which will not be sensational either, so there is a strong impulse to exaggerate and make captivating announcements.

Another difficulty in informing about GM plants and food in the media is connected with the science itself (or more precisely, the scientists themselves). In the last decade, Hungarian scientists have been quarrelling violently, accompanied by lesser or greater publicity, about the benefits and risks of gene technologies as well as the necessity of their authorization or restriction, seemingly without any chance to come to a consensus. These articles published by the experts in public interest magazines, educational and scientific journals, sometimes making personal remarks as well, are - in spite of their small number of great importance in providing information to the public as far as their influence is concerned. Journalists or editors are not competent to judge on a professional basis these disputes between high-ranking eminent representatives of science. At the same time, if they are informed in the subject, they should know about these disputes and they should also know which scientist maintains which view. Thus in case of need they can find prestigious experts for any of the opposing positions. Accordingly, the information provided to the public will depend more on the personal belief of the member of the press than the scientifically proven results. It is possible that no duly established position can be taken yet with regard to the hazards and benefits of GM plants and food. But as long as the professional representatives of these specific areas, who in principle speak the same language, cannot come to at least roughly similar conclusions from the same data, they are somewhat unjustified in blaming media workers for their inadequate expertise and preparedness. Though this may be the point where the opinions of the arguing parties are the most comparable: 
“...success in public life is not necessarily a result of good preparedness. It can be achieved by becoming a spokesperson of a community; or by being referred to as an expert by the rhapsodic media. ... But the opinion of researchers who only appear before other scientists will not be heard. Their messages are shown as the last results in internet searches; their voices fade into the information flood. ... how could I write down how silly the things published in the press are. What makes an uninformed person write an article if he/she is not persevering enough to read the available documents?... And why do the editors give a dilettante remark on this unknown subject? So when can we expect specialist journalists to appear? The answer seems to be 'never' ” (Béla Darvas: Legyen meg az akaratuk - Let their will be done. Élet és Irodalom, Vol. L. No. 39, 29 September 2006)

"Outsiders, such as the major part of the media, thus tend to think that the question is scientifically undetermined; half of the researchers reveal hazards, while the other half do not. But it is a completely false statement, and it is as such due to the nature of the media. Because if anybody in the world announces that the consumption of GM food is hazardous, most of the world press will put it in the headlines immediately. On the contrary, the news on the hundreds of control experiments proving the safety of the technology has no value at all, or if, by any possibility, it is published in a printed matter, the determined opponents of gene engineering would at once accuse the researcher performing the examinations of being the paid agent of the multinational companies interested in the technology." (Pál Venetianer: Féljünk-e a génmanipulált élelmiszerektől? - Should we fear of GM food?, Part One, Természet Világa, Vol. 136, No. 10, October 2005)

Writing about GM issues is, therefore, one of the most difficult journalistic tasks. In spite of the greatest prudence and the most thorough preparation, there is a very good chance that the journalist will gain the disapproval of one of the scientists' groups

\section{Genetically modified crops: A beginning or the end?}

„Much ado about nothing”

BÉLA SOMFAI

The huge opportunities created by the swift development of genetic technology have truly eclipsed the achievements of the Green Revolution of the previous century in the creation of high yield hybrid crops. The mapping of the DNA chain and its components at the molecular level opened the door to identifying the genetic traits of living organisms and to using the tools of genetic engineering to copy genetical information encoding the desired traits into other, naturally not cross-compatible organisms. The hybrids or transgenic organisms express the new traits and pass them on to their progeny. Thus, a fast track of targeted, artificially introduced changes is so opened next to the slow track of random changes and natural selection. The new organisms are playing a significant role in medicine already, and their spread in agriculture will hopefully contribute to the economic development of poor countries and alleviate the food shortages forecast to sharply increase by the middle of this century. The significance of this new technology for humankind is comparable to the impact of computers and the internet.

However, these bright expectations are tainted by dark concerns. Since we are unable to identify either the direction or the goals of natural development, we can not accurately forecast the long-term ramifications of the results achieved. Furthermore, history teaches us that scientific knowledge and technology is a double edged sword. This essay, however, is not intended to deal with the dangers of wilful damage. Rather, it aims to distinguish the concerns and unjustified objections accompanying the agricultural and food processing applications of genetically modified organisms from the rules imposed by moral obligations, and to identify or at least outline the limits of the latter.

As a first step, I will explore the principled standpoints of some the major world religions and movements, and will point out that their objections are based not only on the ideological or moral foundations of these organizations, but also to a large extent on their (mis)understanding of the procedures of gene technology, and their scientifically sound or mistaken interpretation. These communities evaluate genetic engineering and even more its agricultural application by its compatibility with their view of the world, humanity and God, and with their moral values. Usually, a link exists between the sources of faith or ideological convictions and their practical application on one hand, and a community's religious or ideological stance in the more mundane matters of politics and the economy, and so in that of genetic engineering on the other. Therefore religious or ideological convictions, and the resulting notions and prohibition of "unnatural practices" can not be fully separated. For many of these communities and schools of thought the artificial and therefore "unnatural" transmission of 
genetic information between living organisms poses an ethical problem either absolutely, or at least when such transmission takes place among animals or plants used as food. This problem becomes especially acute when genetic information is copied from humans.

Muslims, Hindus and Sikhs believe that it is the human or animal origin of the copied genes which determines whether the products so obtained are ethical or not. Accordingly, the consumption of food containing genetic information from humans or proscribed animals invariably violates both the laws of nature and the dietary laws of these religions, even if the genes were transplanted into plants. Therefore Muslims or Hindus and Sikhs must not even eat food made from plants that contain genetic information from pigs or cattle respectively. Namely, pork is forbidden for Muslims, and beef is forbidden for Hindus and Sikhs. Since Hindus and Sikhs are also required to respect other forms of life, the copying of any genetic information from animals in general into plants renders such plants out of bounds for consumption.

Buddhists maintain that humans must respect and protect all forms of life. Accordingly, they condemn all damage to or extinction of all life forms, even in the name of scientific research or for the purpose of curing illnesses and alleviating suffering. For example, Buddhists disapprove of the sacrificing of embryos for research. They believe all medical or scientific attempts to eliminate suffering are futile, because suffering can only be eliminated by spiritual means. If, and only if, the copying of genetic information into other life forms can be achieved without doing damage, the results eliminate or reduce human suffering and its benefits are equally available to everybody, do Buddhists see no ethical reason to oppose it (Béla Somfai, 2006, "Religious traditions and stem cell research". In: Judit Sándor (ed.) Society and Genetic Information, Codes and Laws in the Genetic Era. CEU Press, Budapest, p. 85.).

The Jewish point of view, although not premised on a uniform consensus, is based on four principles. First, our physical health is a gift from God we must protect, since we are not the owners but only the users and stewards of our body. Second, in our healing activities we partner with God and must use all available natural and artificial means. Third, since God created man in his own image, all men are equal in healing. And fourth, since we can not always foresee the possible consequences of our actions, we must do everything within our power to prevent doing damage. According to Jewish opinion, the ethical significance of artificially copying genetic information is decided not by the source of the information, but by the external appearance of the recipient. As long as a cow's essential characteristics are recognizable (four hoofed feet and rumination, for example), it remains a cow even if it has become the carrier of genetic information derived from animals or plants. We must do everything, however, to eliminate the possibility of doing damage in the short or long term. If these criteria are fulfilled, the majority of Jewish opinion does not oppose the application of gene technology. Just distribution of the benefits and the creation of the right conditions for informed consent are also regarded as important by this religious tradition. Orthodox tradition, however, sets stricter ethical standards by proscribing the crossing of boundaries between species and varieties, and the mixing of their characteristic traits (Leviticus 19:19: "Ye shall keep my statutes. Thou shalt not let thy cattle gender with a diverse kind: thou shalt not sow thy field with mingled seed: neither shall a garment mingled of linen and woollen come upon thee."). This is because this would confuse public opinion and disturb the order of creation. As a result, this tradition accepts the copying of genetic information only in those cases where such copying is also possible in the course of natural selection. It makes an exception, however, for genetic modification (GM) procedures which aim to save lives or are required for the creation of life saving drugs (Béla Somfai, i. m. p. 86.). These or similar opinions are also found in smaller Christian or other religious groups and nature religions which regard the artificial transplantation of genetic information unnatural

These opinions, if not their bases in principle, are shared by some other ideological movements. Vegetarians are not unanimous on this issue. In general, they are also worried by the presence of genetic information of animal origin in their food. They reject the transplantation of human genes into animals or plants. However, they accept GM procedures they deem necessary, such as the use of recombinant rennet in cheese production instead of the natural rennet derived from the dried stomachs of young calves. They also oppose GM procedures performed on animals and the appearance of animal-based genetic information in their food. Animal rights organizations are concerned about the conditions under which GM animals used for drug production are kept. Namely, these animals are kept in a closed and sterile environment which causes them great suffering and prevents them from living a natural life. Therefore they strongly oppose the consumption of products made from GM animals, such as sheep, goats and cows, which were modified to produce drugs in their milk or blood. The Friends of the Earth network denounces the lack of legislated rights for GM animals undergoing untold suffering, such as mice modified to have cancer or pigs raised to huge size with human growth hormone genes. They admit, however, that suitably applied GM procedures do improve the quality of life. The Society Against Vivisection also opposes GM experiments, the consumption of food made from GM materials, and denies that GM procedures are basically identical to breeding based on natural selection. They object to the confidentiality of GM research, which they see as the root of the mistrust of and controversy around biotechnology. Green movements base their opposition on the potential loss of the ecological balance or the possibility that natural animals and plants could be displaced (Basma Ellahi, 1994, British Food Journal, 96: 16.).

The attitude of Christians closely resembles that of the general Jewish public, with no major differences among Roman Catholics, Orthodox Christians and Protestants. Pope John Paul II made repeated pronouncements about the issues surrounding the use of modern biotechnology, and his opinion mirrors the shared tradition. He declared that its use in agriculture, medicine and the protection of the environment created new hopes and worries, and set off far-ranging public debates about the important values involved. 
„The Christian vision of creation makes a positive judgment on the acceptability of human intervention in nature, which also includes other living beings, and at the same time makes a strong appeal for responsibility. ... In effect, nature is not a sacred or divine reality that man must leave alone. Rather, it is a gift offered by the Creator to the human must leave alone. Rather, it is a gift offered by the Creator to the human
community, entrusted to the intelligence and moral judgment of men and women."

The Pope also emphasized that modern biotechnologies have powerful social, economic and political impact locally, nationally and internationally. They need to be evaluated according to ethical criteria... "Above all the criteria of justice and solidarity must be taken into account." (Pontifical Council for Justice and Peace, 2009, Compendium of the Social Doctrine of the Church, par. 473-478.)

The acceptability of the use of biological and biogenetic techniques is only one part of the ethical problem - continued the Pope. As with every human behaviour, it is also necessary to evaluate accurately the real benefits as well as the possible consequences in terms of the risks of technological-scientific interventions that have forceful and widespread impact on living organisms, with the possibility of significant long-term repercussions. In any event, one must avoid falling into the error of believing that only the spreading of the benefits connected with the new techniques of biotechnology can solve the urgent problems of poverty, health risks, agricultural underdevelopment and hunger that still afflict so many countries on the planet. Solidarity requires the just distribution of the goods so produced, making the scientific knowledge and technical-medical means required for the appropriate use of these technologies available to poor countries, and the creation of the right conditions for equitable commercial exchange, without the burden of unjust stipulations. We must not forget - emphasized the Pope - that the knowledge on which these technologies are built, belongs to the patrimony of humanity and is destined also to future generations. For believers, it is a question of a gift received from the Creator, whose just ownership must be extended to developing countries. Entrepreneurs and directors of public agencies involved in the research, production and selling of products derived from new biotechnologies must take into account not only legitimate profit but also the common good. This principle also holds true for the leaders of developing countries. It becomes particularly important for activities that deal with the food supply, medicine, health care and the environment. With these thoughts the Pope emphasized the importance of prudence and precaution, as well as the actions required to safeguard them (i. m. par. 473,474 ).

As shown by this brief summary of relevant opinions, a number of aspects need to be balanced for the evaluation of the ethical issues surrounding the agricultural use of GM technologies. Some of these aspects are related to the visions of God, the world, and humans offered by religious traditions and philosophies, as well as their knowledge of GM technologies. Another group of aspects is related to the global risks and benefits of these technologies. A third, high priority group is comprised of the need to increase food supplies and to ensure the just distribution of the benefits.
Now that we have seen the views and attitudes of key stakeholders, let us examine their objections. According to their themes, these objections fall into four large categories: 1 . The theoretically assumed unnaturalness of gene technology and the religious and ethical objections based on its perceived unsoundness, 2 . potential harm caused to human health and the environment, 3. potential dangers to traditional forms of agriculture, and 4. restrictions imposed on use, to protect the profits of large companies. These objections need to be balanced against the economic and social benefits deriving from the agricultural use of GM technologies, especially in developing countries.

1. In our investigation of the issue of unnaturalness, we need to separate the importance of traits describing humans and human nature from the importance of traits characterizing the organic and inorganic world. Man has a set of basic common traits we can conceptionally grasp that make humans recognizably human. Changing them would make basic human values unachievable, obscure or make uncertain the purpose of human existence, and render humans unrecognizable as human or inhuman. This is one of the oldest tenets of critical thinking. Classical philosophy used these traits to create the concept of human nature. Christian philosophy says their modification would erase God's image in man. Since human development is an ongoing process, a full description of the nature of man is beyond our grasp. We can safely say, however, that our ability to achieve beauty and goodness, understand truth, practice self-determination, etc. does differentiate us from other creatures and that we would not be human without these abilities. This, however, does not mean that the existing and future tools of gene technology should never be used on humans. If anything, these tools can help us manifest our dormant potentials and act with a sense of greater responsibility.

Can we make similar statements for other creatures? Let us think about this for a moment. If we postulate that God has a master plan for his creation, the answer is yes. Meddling with this master plan without understanding it fully would be a reckless endeavour at best. If, however, we think of the world as a random but self-sustaining and evolving system - which our current knowledge does not rule out - then we face a harder challenge answering this question, because, for example, the direction of developments based on random change can not be known accurately. Based on common sense and a Christian world view we can state, however, that GM procedures serving mankind and forestalling the foreseeable dangers with high levels of probability should not be dismissed out of hand. We can even go one step further and make a stronger statement. Assuming that we are the responsible stewards of the Earth, the question we should ask is not whether we are allowed to exercise our options and take justified action, but rather what powerful reasons, if any, should compel us not to act? In evaluating these powerful reasons the foreseeable risks of our actions should be weighed against their potential benefits. Even our thirst for knowledge tells us that we should go and explore the unknown, at the price of taking risks. Humans, however, should not be the first to be exposed to these risks, even though our future depends largely on our ability to maintain the balance of the biosphere. 
Throughout history, mankind has never stopped moulding its environment, including plants and animals. The main difference between traditional selection and the opportunities afforded by modern gene technology lies in the latter's ability to introduce changes in a targeted manner, faster, more accurately and more efficiently. The argument of GM being "unnatural" can be countered with the fact that GM involves the selective transfer of genetic information defining desirable traits and processes, and not the wholesale transplantation of the entire genome of a plant or animal into a different species. In other words, genetic modification has no a priori limits: taking an exercisable option may be evil and irresponsible, but it can also be a responsible, even desirable step. The difference lies in the purpose of acting and the consequences.

2. To address the risk of environmental damage, we need to consider an underlying philosophical issue first. People can owe a debt to existing persons or groups only; we can not be indebted in the narrow sense of the word to people who have not been born yet. So then if debt is not a reliable foundation on which we can build the concept of responsibility for long term consequences, what is a more reliable basis? Classical philosophy gives us the answer in the word "piety" (pietas, in the sense of "mercy" or "dutiful conduct"). The richness of nature is the heritage of the family of mankind, a free gift we must keep in its entirety. Squandering and wasting it would be an irresponsibility in itself with which we would cause the greatest harm to our own quality of being human. This is the foundation upon which we can build a deeper understanding of the 2009 statement of the Pontifical Academy of Sciences using scientific arguments urging us to disseminate and deploy the achievements of GM technologies in agriculture and food processing, and giving use detailed guidelines on how to do it (Pontifical Academy of Sciences, PAS, Study Week, Vatican City, 15 to 19 May 2009).

More than 1 billion people are currently undernourished. The expected addition of 2-2.5 billion people to reach a total of approximately 9 billion people by 2050 adds urgency to this problem. The predicted consequences of climate change and associated decrease in the availability of water for agriculture will also affect our ability to feed the increased world population. Agriculture as currently practiced is unsustainable, as evidenced by the massive loss of topsoil and unacceptably high applications of pesticides throughout most of the world. The appropriate application of GE in agriculture can increase crop yields significantly. There is nothing intrinsic about the use of GE technologies for crop improvement that would cause the plants themselves or the resulting food products to be unsafe. Special efforts should be made to provide poor farmers in the developing world with access to high yield GE crop varieties adapted to their local conditions and technologies, at affordable prices.

3. Examining the impact of GM technologies on agriculture, the PAS established that these technologies have become much more reliable and safer than traditional farming methods. This is proven by the results of countries, such as the USA, China, Argentina, India, and many other developing countries, where pest and weed resistant GM crops (mainly soybean, corn, cotton and rape) are widely grown. Such crops, adapted to local conditions, require less energy, fertilizer and pesticide which is favourable for the ecological balance and human health. In addition, the use of new GM crops also reduces soil erosion, better preserving the balance of the biosphere. This shows that the risks and burdens of GM crops should not be examined in isolation, but rather in comparison with those of traditional varieties.

4. Ensuring the availability and affordability of GM crops is only the first step, however. To use and process them, we need to adapt and develop scientific, technological and business knowledge and tools, and create just distribution systems. At the same time we need to recognize that GM technologies are not able to live up to all of our expectations, and therefore we need to keep alive the traditional methods and tools of agriculture, and keep developing our economic infrastructure as well.

The statement of the PAS points out that according to Christian tradition, the goods of the earth are destined for all mankind. Private and public actors should recognize that the legitimate claims of their property rights should be subordinated to this universal principle and not allow the exploitation of the poor and vulnerable. To ensure the just distribution of food, private and public actors must work together. We see encouraging examples where proprietary technologies are made available for the benefit of the poor and profit motives are subordinated to the interests of the poor, even though most intellectual property rights are held by large international corporations. The cooperation of the public and private sectors creates "a composite reality which does not exclude profit but sees it as a means for attaining human and social ends". This "very plurality of institutional forms gives rise to a market which is not only more civilized but also more competitive." (Pope Benedict XVI, Encyclical Letter Charitas in Veritate, Vatican City, 29 June 2009, par. 46.)

The public sector should not only support research and development, but should also endeavour to make its achievements widely available in agricultural production and health services. At the same time, excessive regulation must be avoided, because this inflates the costs of research and development and hinders social and economic progress benefiting the poor. The excessive and unnecessary regulation of GM technologies makes them too expensive to apply to 'minor' local crops, creating two problems: first, these crops require a lot of investment and generate little profits, and so are squeezed out of international commerce. Second, developers can not achieve returns commensurate with the investment and risk of minor crops, and therefore they abandon these in favour of major commodity crops. Overly stringent regulation was developed by wealthy countries and was focused almost exclusively on the hypothetical risks of GM crops, discriminating against poor countries, as well as against smaller and poorer producers and retailers. The evaluation of new GM crop varieties should be based on the traits of plant varieties and not on the technologies used to produce them. This would facilitate the exploitation of the potentials of the technology for our common benefit by delivering novel varieties, and make their use in poor communities and in the developing world safe, acceptable and useful. We cannot become more risk averse about science and technology - and the consequent 
risks of food and farming - than what we see as acceptable in the rest of our daily lives.

The putative hazards associated with the genetic engineering of crop plants do not differ from those associated with other instances of the application of such genetic engineering to other organisms. Short-term risks arising from the presence of toxic or allergenic products can be studied and excluded from new crop varieties, a procedure that is more precautionary than is usually the case in the cultivation of crop varieties produced by conventional breeding. Our present understanding of molecular evolution clearly shows that genetic modifications engineered into a genome can only follow the well-studied natural strategies of biological evolution. Viable variations can only develop in small steps. This becomes understandable if we bear in mind that plant genomes are like large encyclopedias of several hundred books, while genetic modifications using modern genetic techniques affect only one or a few genes out of about 20 to 30 thousand in the average plant genome. Therefore, the possible evolutionary risks of genetic engineering events cannot be greater than the risks of the natural process of biological evolution or of the application of chemical mutagenesis, both responsible for generating extensive and poorly characterized degrees of genetic change. Statistical records show that the undesirable effects of such genetic change are extremely rare and are more precisely identifiable than in the case of conventional breeding. Activities that deal with the food supply, medicine and health care should enjoy the highest priority. Biotechnologies should be carefully evaluated both in terms of their potential profits and their risks, and the resulting information must be made accessible to everybody, in an objective and easy-to-understand form

In summarizing the relevant opinions, prudence must be employed, even though we know by now that the possible evolutionary risks of genetic engineering events are not greater than the risks of the natural process of biological evolution. Prudence is indicated, because this topic is still mired in controversy, both in scientific research and in the media and public opinion. In fact, we face a lot of high priority work in dispersing alarmist concerns created by sensationalist media. We need a complex evaluation method showing not only risks and the extent of possible damage, but also offering tools to calculate their probabilities and suggesting steps to avoid them. "We need initiatives which create order where scientific uncertainties prevail, and reconcile the differences of opinion and arguments concerning the weight of such uncertainties."(Anne Ingeborg Myhr, 2010, Journal of Agricultural Environment Ethics 23: 503.) As one UNESCO statement published in 2005 says:

When human activity causes morally unacceptable damage which is possible but uncertain, steps must be taken to avoid or reduce it. ... In being prudent, we must bear in mind two factors: 1) the symptoms of damage, and 2) the extent of uncertainty, scientifically calculated, concerning the occurrence and the impact of the damage." (Anne Ingeborg Myhr i. m. p. 505.)
For genetically modified plants and food items, proving safety is a legal obligation. This burden of proof is often a high hurdle to leap. Most countries require the step-by-step application of the regulatory process for each GM plant. This means a gradually extended evaluation of their environmental impact from glass house tests through field trials to market approval. A fully unanimous scientific consensus in the evaluation of test results is not likely to be achieved either. This is to be expected, since the opinion an evaluator forms about the impacts of the observed phenomena on human health or the environment depends on the value hierarchy of that evaluator. Similar differences may arise in judging the importance of the criteria by which the matter at hand is evaluated. Since unknown factors may also play a role (complex environmental systems are also governed by random events), long term monitoring is a necessity - but the imposition of limitations is not. To prove the objectivity of scientific knowledge, we need both to accurately assess the risks and uncertain factors and explore the limits of available knowledge. Disputes and controversies will most probably continue, and will continue to be the most effective means of clarifying outstanding issues.

\section{Further information:}

- Burachik, M. (2010): Experience from use of GMOs in Argentinean agriculture, economy and environment. New Biotechnology, 27: 588-592. doi:10.1016/j.nbt.2010.05.011

- Weale, A. (2010): Ethical arguments relevant to the use of GM crops. New Biotechnology 27:582-587. http://ww.ask-force.org/web/Vatican-PASStudyweek-Elsevier-publ-20101130/Weale-Albert-PAS-Ethical-ArgumentsRelevant-20101130-publ.pdf

- The statement of the Pontifical Academy of Sciences is available for download here: http://www.ask-force.org/web/Vatican-PAS-Statement-FPTPDF/PAS-Statement-English-FPT.pdf

- Gene technology information: http://www.ncbe.reading.ac.uk/ 


\section{Reference list of Hungarian research papers describing GMOs}

\section{6}

- Deák, M., Kiss, Gy. B., Koncz, Cs., Dudits, D. (1986): Transformation of Medicago by Agrobacterium-mediated gene transfer. Plant Cell Reports, 5: $97-100$

1991

- Fehér, A., Felföldi, K, Preiszner, J., Dudits, D. (1991): PEG-mediated transformation of leaf protoplasts of Solanum tuberosum L. cultivars. Plant Cell, Tissue and Organ Culture, 27: 105-114

1992

- Fehér, A., Skryabin, K.G., Balázs, E., Preiszner, J., Shulga, O.A., Zakharyev, V. M., Dudits, D. (1992): Expression of PVX coat protein gene under the control of extensin-gene promoter confers virus resistance on transgenic potato plants. Plant Cell Reports, 11: 48-52.

1993

- Dudits, D., Mórocz, S., Omirulleh, S. (1993): Transgenic maize plants from protoplasts: new products for plant breeding. Hungarian Agriculture Research, 2/1: 4-8.

- Golovkin, V. M., Ábrahám, M., Mórocz, S., Bottka, S., Fehér, A., Dudits, D. (1993): Production of transgenic maize plants by direct DNA uptake into embryogenic protoplasts. Plant Science, 90: 41-52.

- Kollár, Ä., Thole, V., Dalmay, T., Balázs, E. (1993): Efficient coat protein mediated cross protection induced by integrated potato virus $Y$ coat protein gene in tobacco. Biochemie, 75: 623-629.

- Omirulleh, S., Ábrahám, M., Golovkin, M., Stefanov, I., Karabaev, M., Mustárdy, L., Mórocz, S., Dudits, D. (1993): Activity of a chimeric promoter with the doubled CaMV $35 \mathrm{~S}$ enhancer element in protoplast-derived cells and transgenic plants in maize. Plant Molecular Biology, 21: 415-428.

$$
1994
$$

- Aszódi, A., Módis L., Páldi A., Altanchimeg R., Kiss I., Bôsze Zs. (1994): The zonal expression of chicken cartilage matrix protein gene in the developing skeleton of transgenic mice. Matrix Biology, 14: 181-190.

- Stefanov, I., Fekete, S., Bögre, L., Pauk, J., Dudits, D. (1994): Differential activity of the mannopine synthase and the CaMV 35 S promoters during development of transgenic rapeseed plants. Plant Science, 95: 175-186.

$$
1995
$$

- Fári, M., Nagy, I., Csányi, M., Mitykó, J., Andrásfalvy, A. (1995): Agrobacteriummediated genetic transformation and plant regeneration via organogenesis and somatic embryogenesis from cotyledon leaves in eggplant (Solanum melongena L. cv. Kecskeméti lila). Plant Cell Reports, 15: 82-86.

- Palkovics, L., Wittner, A., Balázs, E. (1995): Pathogen-derived resistance induced by integrated plum pox virus coat protein gene into plants of Nicotiana benthamiana. Acta Horticulturae, 386: 311-317.
- Pauk, J., Stefanov, I., Fekete, S., Bögre, L., Karsai, I., Fehér, A., Dudits, D. (1995): A study of different ( CaMV $35 S$ and mas) promoter activities and risk assessment of field use in transgenic rapeseed plants. Euphytica, 85: 411416.

\section{6}

- Baranyi, M., Aszódi, A., Devinoy, E., Fontaine, M-L., Houdebine, M-L., Bôsze, Zs. (1996): Cloning of rabbit kappa casein gene and in vivo expression in transgenic mice. Gene, 174: 27-34.

- Jenes, B., Bittencourt, P. A. L., Csányi, A., Pauk, J., Nagy, I., Toldi, O., Balázs, E. (1996): The GENEBOOSTER - a new microparticle bombardment device - for genetic transformation of plants. Plant Tissue Culture and Biotechnology, 2: 42-51.

- Szász, A., Mitykó, J., Andrásfalvy, A., Fári, M. (1996): Methodological and genetic aspects of in vitro plant regeneration and genetic transformation of the recalcitrant pepper (Capsicum annuum L.). Acta Horticulturae, 447: 365-366.

1998

- Giczey, G., Kerényi, Z., Dallmann, G., Hornok, L. (1998): Homologous transformation of Trichoderma hamatum with an endochitinase encoding gene, resulting in increased levels of chitinase activity. FEMS Microbiology Letters, 165: 247-252.

- Hornok, L., Giczey, G., Kerényi, Z. (1998): Strain improvement in biocontrol fungi. Phytoprotection, 79: 136-138.

- Katarova, Z., Mugniani, E., Sekerkova, G., Mann, J., Aszódi, A., Bősze, Zs., Greenspan, R., Szabó, G. (1998): Regulation of cell-type specific expression of lac $Z$ by the $5^{\prime}$-flanking region of mouse GAD67 gene in the central nervous system of transgenic mice. European Journal of Neuroscience, 10: 989-999.

- Szász, A., Szilassy, D., Salánki, K., Fári, M., Balázs, E. (1998): A simple and efficient method for the transformation of eggplant (Solanum melongena L.). Acta Agronomica Hungarica, 46: 201-207.

- Wittner, A., Palkovics, L., Balázs, E. (1998): Nicotiana benthamiana plants transformed with the plum pox virus helicase gene are resistant to virus infection. Virus Research, 53: 97-103.

\section{9}

- Aigner, B., Pambalk, K., Reichart, U., Besenfelder, U., Bôsze, Zs., Renner, M., Günzburg, W. H., Wolf, E., Müller, M., Brem, G. (1999): Species-specific alternative splicing of transgenic RNA in the mammary glands of pigs, rabbits and mice. Biochemical and Biophysical Research Communications, 257: 843-850

- Aszódi, A., Bateman, J., Hirsch, E., Baranyi, M., Hunziker, E. B., Bôsze, Zs., Fässler, R. (1999): Normal skeletal development of mice lacking matrilin1: redundant function of matrilins in cartilage. Molecular Cellular Biology, 19: $7841-7845$. 
- Bilgin, M., Dedeoglu, D., Omirulleh, S., Peres, A., Engler, G., Inzé, D., Dudits, D., Fehér, A. (1999): Meristem and cell division-dependent activity of wheat histone $\mathrm{H} 4$ promoter can be modified by growth regulators in transgenic maize plants. Plant Science, 143: 35-44.

- Bôsze, Zs., Baranyi, M., Hiripi, L., Fontaine, M-L., Devinoy, E. (1999): High level expression of rabbit kappa casein in the milk of transgenic mice. Transgenic Research, 8: 56.

- Gerencsér, Á., Hiripi, L., Makovics F., Tóth Sz., Szabó G., Bôsze Zs., Arányi P. (1999): Transgenic animals for in vivo testing of potential anti-amnesic therapeutic agents. Fundamental and Clinical Pharmacology, 13: Supplement 1., 327.

- Horváth, G. V., Oberschall, A., Deák, M., Sass, L., Vass, I., Barna, B., Király, Z., Hideg, E., Fehér, A., Dudits, D. (1999): Transgenic strategy to improve stress resistance of crop plants. Journal of Plant Biotechnology, 1: 61-68.

- Kiss, E., Veres, A., Varga, Á., Galli, Zs., Heszky, L. E., Tóth, E., Hrazdina, G. (1999): Genetic transformation of carnation to downregulate ethylene biosynthesis. In Vitro Cellular and Developmental Biology - Plant, 35: 174-175.

- Peres, A., Nikovics, K., de Almeida-Engler, J., Engler, G., Inzé, D., Fehér, A., Dudits, D. (1999): An Arabidopsis cyclin promoter region is active in transgenic maize plants. Cereal Research Communications, 27: 223-230.

- Pónya, Z., Finy, P., Fehér, A., Mitykó, J., Dudits, D., Barnabás, B. (1999): Optimisation of introduction foreign genes into eggs cells and zygotes of wheat (Triticum aestivum $\mathrm{L}$.) via microinjection. Protoplasma, 208: 163-172.

- Xue, B., Ling, K-S., Reid, C.L., Krastanova, S., Sekiya, M., Momol, E.A., Süle, S., Mozsár, J., Gonsalves, D., Burr, T. J. (1999): Transformation of five grape rootstocks with plant virus genes and a virE2 gene from Agrobacterium tumefaciens. In vitro Cellular and Developmental Biology - Plant, 35: 226231

\section{0}

- Hideg, É., Oberschall, A., Horváth, G.V., Vass, I., Dudits, D. (2000): Enhanced oxidative stress tolerance in transgenic tobacco. Plant Physiology and Biochemistry, 38: 234.

- Hiripi, L., Baranyi, M., Szabó, L., Tóth, Sz., Fontaine, M-L., Devinoy, E. M., Bôsze, Zs. (2000): Effect of rabbit kappa-casein expression on the properties of milk from transgenic mice. Journal of Dairy Research, 67: 541-550.

- Kiss, E., Veres, A., Galli, Z., Nagy, N., Tóth, E., Varga, Á., Hrazdina, G., Heszky, L. (2000): Production of transgenic carnation with antisense ACS (1aminocyclopopane-1-carboxylate synthase) gene. International Journal of Horticultural Science, 6: 103-107.

- Mihálka, V., Fári, M., Szász, A., Balázs, E., Nagy, I. (2000): Optimised protocol for efficient plant regeneration and gene transfer in pepper (Capsicum annuum L.). Journal of Plant Biotechnology, 2: 143-149.
- Oberschall, A., Deák, M., Török, K., Sass, L., Vass, I. Kovács, I., Fehér, A., Dudits, D., Horváth, V. G. (2000): A novel aldose/aldehyde reductase protects transgenic plants against lipid peroxidation under chemical and drought stresses. The Plant Journal, 24: 437-446.

- Szabó, Z., Bánfalvi, Zs. (2000): An Agrobacterium-mediated transformation system for the tomato cultivar "Kecskeméti 262". Acta Agronomia Hungarica, 48: 221-226.

\section{1}

- Kehm, R., Jakob, N. J., Welzel, T. M., Tobiasch, E., Viczián, O., Jock, S., Geider, K., Süle, S., Darai, G. (2001): Expression of immunogenic Puumala Virus nucleocapsid protein in transgenic tobacco and potato plants. Virus Genes, 22: 73-83.

- Molnár, A., Lovas, Á., Bánfalvi, Zs., Lakatos, L., Polgár, Z., Horváth, S. (2001): Tissue-specific signal(s) activate the promoter of a metallocarboxy peptidase inhibitor gene family in potato tuber and berry. Plant Molecular Biology, 46: 301-311.

- Rakszegi, M., Tamás, C., Szúcs, P., Tamás, L., Bedô, Z. (2001): Current status of wheat transformation. Journal of Plant Biotechnology, 3: 67-81.

- Sakai, K., Hiripi, L., Glumoff, V., Brandau, O., Vuorio, E., Bôsze, Zs., Fässler, R., Aszódi, A. (2001): Stage and tissue-specific expression of a Col2a1-Cre fusion gene in transgenic mice. Matrix Biology, 19: 761-767.

2002

- Dóczi, R., Csanaki, C., Bánfalvi,Zs. (2002): Expression and promoter activity of the desiccation-specific DS2 gene of potato (Solanum tuberosum L.). Plant, Cell \& Environment, 25: 1197-1203.

- Józsa, R., Stasevski, Z., Wolf, I., Horváth, S., Balázs, E. (2002): Potato virus $Y$ coat protein gene induced resistance in valuable potato cultivars. Acta Phytopathologica et Entomologica Hungarica, 37: 1-7.

- Józsa, R., Stasevski, Z., Balázs, E. (2002): High level of field resistance of transgenic tobaccos induced by integrated potato virus $\mathrm{Y}$ coat protein gene. Acta Phytopathologica et Entomologica Hungarica, 37: 311-316.

- Kelemen, Zs., Mai, A., Kapros, T., Fehér, A., Györgyey, J., Waterborg, J. H., Dudits, D. (2002): Transformation vector based on promoter and intron sequences of a replacement histone $\mathrm{H} 3$ gene. A tool for high, constitutive gene expression in plants. Transgenic Research, 11: 69-72.

- Khattak, S., Darai, G., Süle, S., Rosen-Wolff, A. (2002): Characterization of expression of Puumala viris nucleocapsid protein in transgenic plants. Intervirology, 45: 334-339.

- Mészáros, A., Pauk,J. (2002): Chlorate resistance as a tool to study the effect of nitrate reductase antisense gene in wheat. Cereal Research Communications, 30: 245-252.

- Silhavy, D., Molnár, A., Lucioli, A., Szittya, Gy., Hornyik, C., Tavazza, M., Burgyán, J. (2002): A viral protein suppresses RNA silencing and binds silencing generated 21-25 nt double-stranded RNAs. EMBO Journal, 21: 3070-3080. 
- Süle, S., Kiss, E., Kim, W.-S., Geider, K. (2002): Transformation of SR1 tobacco and JTE-H apple rootstock with the EPS-depolymerase gene from an Erwinia amylovora phage. Acta Horticulturae, 590: 407-409.

- Szabados, L., Kovács, I., Oberschall, A., Ábrahám, E., Kerekes, I., Zsigmond, L., Nagy, R., Alvarado, M., Krasovskaja, I., Gál, M., Berente, A., Rédei, G. P., Ben-Haim, A., Koncz, Cs. (2002): Distribution of 1000 sequenced T-DNA tags in the Arabidopsis genome. Plant Journal, 32: 233-242.

$$
2003
$$

- Ábrahám, E., Rigó, G., Székely, G., Nagy, R., Koncz, Cs., Szabados, L. (2003): Light-dependent induction of proline biosynthesis by abscisic acid and salt stress is inhibited by brassinosteroid in Arabidopsis. Plant Molecular Biology, 51: 363-372.

- Bôsze, Zs., Hiripi, L., Carnwath, J. W., Niemann, H. (2003): The transgenic rabbit as model for human diseases and as a source of biologically active recombinant proteins. Transgenic Research, 12: 541-553.

- Galli, Zs., Kiss, E., Hrazdina, G., Heszky, L. (2003): The effects of ACS (1aminocyclopropane-1-carboxylate synthase) gene down regulation on ethylene production and fruit softening in transgenic apple. International Journal of Horticultural Science, 9: 65-70.

- Hideg, É., Nagy, T., Oberschall, A., Dudits, D., Vass, I. (2003): Detoxification function of aldose/aldehyde reductase during drought and UV-B (280$320 \mathrm{~nm}$ ) stresses. Plant Cell and Environment, 26: 513-522.

- Hiripi, L., Makovics, F., Halter, R., Baranyi, M., Paul, D., Carnwath, J. W., Bôsze, Zs., Niemann, H. (2003): Expression of human blood clotting factor VIII in the mammary gland of transgenic rabbits. DNA and Cell Biology, 22: $41-45$.

- Lovas, A., Bimbó, A., Szabó, L., Bánfalvi, Zs. (2003): Antisense repression of StubGAL83 affects root and tuber development in potato. Plant Journal, 33: 139-147.

- Picoli, E. A. de T., Brommonschenkel, S. H., Cecon, P. R., da Silva, D. J. H, Fári, M., Otoni, W. C. (2003): Study of genetic transformation efficiency via organogenesis and embryogenesis in eggplant (Solanum melongena L. cv. Embú): effects of co-culture, temperature and canamycin and hygromycin-based selection procedures. International Journal of Horticultural Science, 8: 15-23.

- Szittya, G., Silhavy, D., Molnár, A., Havelda, Z., Lovas, A., Lakatos, L., Bánfalvi, Zs., Burgyán, J. (2003): Low temperature inhibits RNA silencing-mediated plant defense by the control of small interfering RNA generation. EMBO Journal, 22: 633-640.

2004

- Alvarado, M., Zsigmond, L., Kovács, I., Cséplô, Á., Koncz, Cs., Szabados, L. (2004): Luciferase gene trapping in Arabidopsis: tagging of stressresponsive genes. Plant Physiology, 134: 1-10.
- Ané, J. M., Kiss, G. B., Riely, B. K., Penmetsa, R. V., Oldroyd, G. E. D., Ayax, C., Lévy, J., Debellé, F., Baek, J. M., Kaló, P., Rosenberg, C., Roe, B. A., Long, S. R., Dénarié, J., Cook, D. R. (2004): Medicago truncatula DMI1 required for bacterial and fungal symbioses in legumes. Science, 303: 1364-1367.

- Bodó, Sz., Gócza, E., Révay, T., Hiripi, L., Carstea, B., Kovács, A., Bodrogi, L., Bôsze, Zs. (2004): Production of transgenic chimeric rabbits and transmission of the transgene through the germline. Molecular Reproduction and Development, 68: 435-440.

- Fodor, B. D., Kovács, Á. T., Csáki, R., Hunyadi-Gulyás, É., Klement, É., Maróti, G., Mészáros, L. S., Medzihradszky, K. F., Rákhely, G., Kovács K. L. (2004): Modular broad-host-range expression vectors for single protein and protein complex purification. Applied and Environmental Microbiology, 70: 712-721.

- Hegedús, A., Erdei, S., Janda, T., Tóth, E., Horváth, G., Dudits, D. (2004): Alfalfa aldose/aldehyde reductase overproducing transgenic tobacco plants show a higher tolerance to low temperature and cadmium stresses. Plant Science, 166: 1329-1333

- Karcagi I., Rauch T., Hiripi L., Rentsendorj O., Nagy A., Bốsze Zs., Kiss I. (2004): Functional analysis of the regulatory regions of the matrilin-1 gene in transgenic mice reveals modular arrangement of tissue-specific control elements. Matrix Biology, 22: 605-618.

- Oreifig, A. S., Kovács, G., Jenes, B., Kiss, E., P. Scott, Toldi, O. (2004): Development of a non-lethal selection system by using the aadA marker gene for efficient recovery of transgenic rice (Oryza sativa L.). Plant Cell Reports, 22: 490-496.

- Palkovics, L., Kryldakov, R., Nádudvari, J., Józsa, R., Balázs, E. (2004): Sequence variants of potato virus $\mathrm{Y}$ in field grown tobacco with different resistance background including transgenic ones. Acta Phytoptahologoica et Entomologica Hungarica, 39: 315-324.

- Pogány, M., Koehl, J., Heiser, I., Elstner, E.F., Barna, B. (2004): Juvenility of tobacco induced by cytokinin gene introduction decreases susceptibility to Tobacco necrosis virus and confers tolerance to oxidative stress. Physiological and Molecular Plant Pathology, 65: 39-47.

- Seregélyes, Cs., Igambergiev, A. U., Maassen, A., Hennig, J., Dudits, D., Hill, R. D. (2004): NO-degradation by alfalfa class 1 hemoglobin (Mhb1): a possible link to PR-1a gene expression in Mhb1-overproducing tobacco plants. FEBS Letters, 571: 61-66.

- Tóth, F., Árpás, K., Szekeres, D., Kádár, F., Szentkirályi, F., Szénási, A., Kiss, J. (2004): Spider web survey or whole plant visual sampling? Impact assessment of $\mathrm{Bt}$ corn on non-target predatory insects with two concurrent methods. Environmental Biosafety Research, 3: 225-31.

- Whitelaw, C. B. A., Hiripi, L., Farini, E., Kastanis, P., Edgar, M., Bốsze, Zs. (2004): On the use of post-transcriptional processing elements into transgenes. Transgenic Research, 13: 75-79. 


\section{5}

- Bittsánszky, A., Kômíves, T., Gullner, G., Gyulai, G., Kiss, J., Heszky, L., Radimszky, L., Rennenberg, H. (2005): Ability of transgenic poplars with elevated glutathione content to tolerate Zinc(2+) stress. Environment International, 31: 251-254

- Bôsze, Zs. (2005): The First International Conference on Transgenic Rabbits. Letter to the Editor. Transgenic Research, 14: 799.

- Devinoy, E., Montoliu, L., Baranyi, M., Thépot, D., Hiripi, L., Fontaine, M-L., Bodrogi, L., Bôsze, Zs. (2005): Analysis of the efficiency of the rabbit whey acidic protein gene 5' flanking region in controlling the expression of homologous and heterologous linked genes. Journal of Dairy Research, 72: 113-119.

- Gyulai, G., Humphreys, M., Bittsánszky, A., Skøt, K., Kiss, J., Skøt, L., Gullner, G., Heywood, S., Szabó, Z., Lovatt, A., Radimszky, L., Roderick, H., Abberton, M., Rennenberg, H., Kómíves, T., Heszky, L. (2005): AFLP analysis and improved phytoextraction capacity of transgenic gshI-poplar clones (Populus canescens L.) in vitro. Zeitschrift für Naturforschung, 60: 300-306.

- Kaló, P., Gleason, C., Edwards, A., Marsh, J., Mitra, R. M., Hirsch, S., Jakab, J., Sims, S., Long, S. R., Rogers, J., Kiss, G. B., Downie, J. A., Oldroyd, G. E. D. (2005): Nodulation signaling in legumes requires NSP2, a member of the GRAS family of transcriptional regulators. Science, 308: 1786-1789.

- Kondrák, M., Kutas, J., Szenthe, B., Patthy, A., Bánfalvi, Zs., Nádasy, M., Gráf, L., Asbóth, B. (2005): Inhibition of Colorado potato beetle larvae by a locust proteinase inhibitor peptide expressed in potato. Biotechnology Letters, 27: 829-834.

- Rakszegi, M. Békés, F., Láng, L., Szúcs, P., Tamás, L., Shewry, P. R., Bedô, Z. (2005): Technological quality of transgenic wheat expressing an increased amount of a HMW subunit of glutenin. Journal of Cereal Science, 42: 1523.

- Simon-Sarkadi, L., Kocsy, G., Várhegyi, Á., Galiba, G., de Ronde, J. A. (2005): Genetic manipulation of proline accumulation influences the concentrations of other amino acids in soybean subjected to simultaneous drought and heat stress. Journal of Agricultural and Food Chemistry, 53: $7512-7517$

- Sós-Hegedús, A., Lovas, A., Kondrák, M., Kovács, G., Bánfalvi, Zs. (2005): Active RNA silencing at low temperature indicates distinct pathways for antisense-ediated gene-silencing in potato. Plant Molecular Biology 59: 595-602.

- Veres, A., Kiss, E., Tóth, E., Tóth, Á., Heszky, L. (2005): Down-regulation of ethylene biosynthesis in carnation (Dianthus caryophyllus L.) by an apple derived ACC-cDNA. International Journal of Horticultural Science, 11: 101-104.

\section{6}

- Bakonyi, G., Szira, F., Kiss, I., Villányi, I., Seres, A., Székács, A. (2006): Preference tests with collembolas on isogenic and Bt-maize. European Journal of Soil Biology, 42: S132-S135.

- Bittsánszky, A., Gyulai, G., Humphreys, M., Gullner G., Csintalan, Zs., Kiss, J., Szabó, Z., Lágler, R., Tóth, Z., Rennenberg, H., Heszky, L., Kőmíves, T. (2006): RT-PCR analysis and stress response capacity of transgenic gshIpoplar clones (Populus $\mathrm{x}$ canescens) in response to paraquat exposure. Zeitschrift für Naturforschung, 61: 699-703.

- Bốsze, Zs., Houdebine, L. M. (2006): Application of rabbits in biomedical research: a review. World Rabbit Science, 14: 1-14.

- Horváth-Szanics, E.,Szabó,Z., Janáky,T.,Pauk,J.,Hajós, Gy.(2006): Proteomics as an emergent tool for identification of stress-induced protein in control and genetically modified wheat lines. Chromatographia, 63: 43-47.

- Kondrák, M., van der Meer, I., Bánfalvi, Zs. (2006): Generation of marker-and backbone-free transgenic potatoes by site-specific recombination and a bifunctional marker gene in a non-regular one-border Agrobacterium transformation vector. Transgenic Research, 15: 729-737.

- Kovács, K. L., Maróti, G., Rákhely, G. (2006): A novel approach forbiohydrogen production. International Journal of Hydrogen Energy, 31: 1460-1468.

- Nagy, A., Baráth, Á., Pauk, J., Gelencsér, É. (2006): Nutritional evaluation of the proteins of broad range herbicide resistant spring wheat lines (Triticum aestivum L.) I. Protein quality. Acta Alimentaria, 35: 355-362.

- Simon-Sarkadi, L., Kocsy, G., Várhegyi, Á., Galiba, G., de Ronde, J. A. (2006): Stress-induced changes in the free amino acid composition in transgenic soybean plants having increased proline content. Plant Biology, 50: 793796.

- Simon-Sarkadi, L., Kocsy, G., Várhegyi, Á., Galiba, G., de Ronde, J. A. (2006): Effect of drought stress at supraoptimal temperature on polyamine concentrations in transgenic soybean having increased proline levels. Zeitschrift für Naturforschung, 61: 833-839.

- Szőke, A., Kiss, E., Toldi, O., Heszky, L. (2006): Production of transgenic carnation with a heterologue 6-phosphofructo-2-kinase/fructose 2,6bisphosphatase bifunctional enzyme cDNA. International Journal of Horticultural Science, $12: 75-79$.

- Zhiponova, M., Pettkó-Szandtner, A., Stelkovics, É., Neer, Zs., Bottka, S., Krenács, T., Dudits, D., Fehér, A., Szilák, L. (2006): Mitosis-specific promoter of the alfalfa cyclin-dependent kinase gene (Medsa;CDKB2;1) is activated by wounding and ethylene in a non-cell division-dependent manner. Plant Physiology, 140: 693-703.

2007

- Baranyi, M., Hiripi, L., Szabó, L., Catunda, A. P., Harsányi, I., Komáromy, P., Bôsze, Zs. (2007): Isolation of functional low-phenylalanine kappa casein expressed in the milk of transgenic rabbits. Journal of Biotechnology, 128: 383-392. 
- Bender, B., Bodrogi, L., Mayer, B., Schneider, Z., Zhao, Y., Hammarstöm, L., Eggen, A., Kacskovics, I., Bôsze, Zs. (2007): Position independent and copy-number-related expression of the bovine neonatal Fc receptor $\alpha$-chain in transgenic mice carrying a $102 \mathrm{~kb}$ BAC genomic fragment. Transgenic Research, 16: 613-27.

- Bittsánszky, A., Gyulai, G., Malone, R. P., Gullner, G., Kiss, J., Czakó, M., Márton, L., Heszky, L., Kómíves, T. (2007): Triggering of a plant molecular defense mechanism; gene expression levels of transgene gshI and poplar gene gsh 1 (Populus $\mathrm{x}$ canescens) in response to the DNA demethylating drug DHAC - an qRT-PCR analysis. Acta Phytopathologica et Entomologica Hungarica, 42: 235-243.

- Bukovinszki, Á., Divéki, Z., Csányi, M., Palkovics, L., Balázs, E. (2007): Engineering resistance to PVY in different potato cultivars in a markerfree transformation system using a "shooter mutant" A. tumefaciens. Plant Cell Reports, 26: 459-65.

- Carstea, B. V., Catunda, A. P., Ilie, D., Varga, L., Bodó, Sz., Kovács, A., Bốsze, Zs., Gócza, E. (2007): Production of mouse twins and triplet of predicted gender. Cloning and Stem Cells, 9: 247.

- Halász, Á., Horváth-Szanics, E., Nagy-Gasztonyi, M., Pauk, J., Hajós, Gy. (2007): Traceability of enzyme activities and immune reactivity of albuminglobulin proteins of wide-range herbicide resistant transgenic wheat lines. Cereal Research Communications, 35: 1405-1413.

- Kocsy, G., Simon-Sarkadi, L., Galiba, G., de Ronde, J. A. (2007): Transformation of soybean and use of transgenic lines in basic and applied research. Transgenic Plant Journal, 1: 129-144.

- Kondrák,M.,van der Meer,I.M.,Bánfalvi,Zs.(2007):Generation of marker-free transgenic plants. ISB News Report, April: 10-11.

- Lang, A., Lanber, E., Darvas, B. (2007): Early-tier tests insufficient for GMO risk assessment. Nature Biotechnology, 25: 35-36.

- Oszvald, M., Kang, T. J., Tömösközi, S., Tamás, C., Tamás, L., Kim, T. G., Yang, M. S. (2007): Expression of a synthetic neutralizing epitope of porcine epidemic diarrhea virus fused with synthetic B subunit of $E$. coli heat labile enterotoxin in rice endosperm. Molecular Biotechnology, 35: 215-224.

- Oszvald, M., Jenes, B., Tömösközi, S., Békés, F., Tamás, L. (2007): Expression of the 1Dx5 High Molecular Weight glutenin subunit protein in transgenic rice. Cereal Research Communications, 35: 1543-1549.

- Oszvald, M., Kang, T. J., Jenes, B., Kim, T. G., Tamás, L., Yang M. S. (2007): Synthesis and assembly of Escherichia coli heat-labile enterotoxin B subunit in transgenic rice (Oryza sativa L.) Biotechnology and Bioprocess Engineering, 12: 676-683.

- Stiller, I., Dancs, G., Hesse, H., Hoefgen, R., Bánfalvi, Zs. (2007): Improving the nutritive value of tubers: Elevation of cysteine and glutathione contents in the potato cultivar White Lady by marker-free transformation. Journal of Biotechnology, 128: 335-343.
- Sző́ke, A., Kiss, E., Veres, A., Kerepesi, I., Tóth, Á., Tóth, E., Toldi, O., Heszky, L. (2007): Biotechnológiai módszerek alkalmazása a szegfú nemesítésében. Kertgazdaság, 39: 69-77.

\section{8}

- Balázs, E., Bukovinszki, Á., Csányi, M., Csilléry, G., Divéki, Z., Nagy, I., Mitykó, J., Salánki, K., Mihálka, V. (2008): Evaluation of a wide range of pepper genotypes for regeneration and transformation with an Agrobacterium tumefaciens shooter strain. South African Journal of Botany, 74: 720-725.

- Barna, B., Smigocki, A. C., Baker, J. C. (2008): Transgenic production of cytokinin suppresses bacterially induced HR symptoms and increases antioxidative enzyme levels in Nicotiana. Phytopathology, 98: 1242-1247.

- Bodrogi, L., Brands, R., Raaben, W., Seinen, W., Baranyi, M., Fiechter, D., Bôsze, Zs. (2006): High level expression of tissue non-specific alkaline phophatase in the milk of transgenic rabbits. Transgenic Research, 15. 627-636.

- Catunda, A. P., Gócza, E., Carstea, V. B., Hiripi, L., Hayes, H., Rogel-Gaillard, C., Bertaud, M., Bôsze, Zs. (2008): Characterization, chromosomal assignment and role of LIFR in early embryogenesis and stem cell establishment of rabbit. Cloning and Stem Cells, 10: 523-34.

- Dancs, G., Kondrák, M., Bánfalvi, Zs. (2008): The effects of enhanced methionine synthesis on amino acid and anthocyanin content of potato tubers. BMC Plant Biology, 8: 65.

- Éva, Cs., Csóti, I., Tamás, L. (2008): Agrobacterium-mediated barley transformation. Acta Biologica Szegediensis, 52: 49-51.

- Hegedús, A., Janda, T., Horváth, V. G., Dudits, D. (2008): Accumulation of overproduced ferritin in the chloroplast provides protection against photoinhibition indiced by low temperature in tobacco plants. Journal of Plant Physiology, 165: 1647-1651.

- Kálai, K., Mészáros, A., Dénes, F., Balázs, E. (2008): Comparative study of constitutive and inducible promoters in tobacco. South African Journal of Botany, 74: 313-319.

- Kars, G., Gündüz, U., Rákhely, G., Yücel, M., Eroglu, I., Kovács K. L. (2008): Improved hydrogen production by uptake hydrogenase deficient mutant strain of Rhodobacter sphaeroides O.U.001. International Journal of Hydrogen Energy, 33: 3056-3060.

- Kisgyörgy, B.N., Tamás, C., Rakszegi, M., Sági, L., Láng, L., Bedô, Z. (2008): Regeneration ability of wheat (Triticum aestivum L.) embryos after bombardment with a particle gun. Acta Biologica Szegediensis, 52: 127130.

- Nagy, A., Pauk, J., Takács, K., Gelencsér, É. (2008): Nutritional evaluation of the proteins of broad range herbicide resistant spring wheat (Triticum aestivum L.) lines. II. Resistance to digestion of marker protein in rat model. Acta Alimentaria 37: 159-166 
- Oszvald, M., Gárdonyi, M., Tamás, C., Takács, I., Jenes, B., Tamás, L. (2008): Development and characterization of a chimaeric tissue specific promoter in wheat and rice endosperm. In Vitro Cellular and Developmental Biology - Plant, 44: 1-7.

- Oszvald, M., Kang, T. J., Jenes, B., Kim, T. G., Tamás, L., Yang, M. S. (2008): Expression of cholera toxin B subunit in transgenic rice endosperm. Molecular Biotechnology, 40: 261-268.

- Papdi, Cs., Ábrahám, E., Joseph, M.P., Popescu, C., Koncz, Cs., Szabados, L. (2008): Functional identification of Arabidopsis stress regulatory genes using the controlled cDNA Overexpression System COS. Plant Physiology, 147: 528-542.

- Rakszegi, M., Pastori, G., Jones, H. D., Békés, F., Butow, B., Láng, L., Bedô, Z., Shewry, P. R. (2008): Technological quality of field grown transgenic lines of commercial wheat cultivars expressing the 1Ax1 HMW glutenin subunit gene. Journal of Cereal Science, 47:310-321.

- Sági, L., Rakszegi, M., Spitkó, T., Mészáros, K., Németh-Kisgyörgy, B., Soltész, A., Szira, F., Ambrus, H., Mészáros, A., Galiba, G., Vágúifalvi, A., Barnabás, B., Marton, L. C. (2008): Genetic modification of cereals in the Agricultural Research Institute of the Hungarian Academy of Sciences. Acta Agronomica Hungarica, 56: 443-448

- Stiller, I., Dulai, S., Kondrák, M., Tarnai, R., Szabó, L., Toldi, O., Bánfalvi, Zs. (2008): Effects of drought on water content and photosynthetic parameters in potato plants expressing the trehalose-6-phosphate synthase gene of Saccharomyces cerevisiae. Planta, 227: 299-308.

- Székely, Gy., Ábrahám, E., Cséplô, Á., Rigó, G., Zsigmond, L., Csiszár, J., Ayaydin, F., Strizhov, N., Jásik, J., Schmelzer, E., Koncz, Cs., Szabados, L. (2008): Duplicated P5CS genes of Arabidopsis play distinct roles in stress regulation and developmental control of proline biosynthesis. Plant Journal, 53: 11-28.

- Zsigmond, L., Rigó, G., Székely, Gy., Ötvös, K., Szarka, A., Darula, Zs., Medzihradszky, K.F., Koncz, Cs., Koncz, Zs., Szabados, L. (2008): Arabidopsis PPR40 connects abiotic stress responses to mitochondrial electron transport. Plant Physiology, 146: 1721-1737.

$$
2009
$$

- Bedô, Z Rakszegi, M., Láng, L (2009): Design and management of field trials of transgenic cereals. In: Methods in Molecular Biology, Transgenic wheat, barley and oats (eds. H. D. Jones and P. R. Shewry) Production and characterization protocols, 478: 305-315.

- Bittsánszky, A., Gyulai, G., Gullner, G., Kiss, J., Szabó, Z., Kátay, Gy., Heszky, L., Kômíves, T. (2009): In vitro breeding of grey poplar (Populus $\mathrm{x}$ canescens) for phytoremediation purposes. Journal of Chemical Technology \& Biotechnology 84: 890-894.

- Kiss, E., Oláh, B., Kaló, P., Morales, M., Heckmann, A. B., Borbola, A., Lozsa, A., Kontár, K., Middleton, P., Downie, J. A., Oldroyd, G. E. D., Endre, G. (2009): LIN, a novel type of U-Box/WD40 protein, controls early infection by Rhizobia in Legumes. Plant Physiology, 151: 1239-1249.
- Lauber, É., Darvas, B. (2009): Increased mortality of isolated first instar larvae of Inachis io (Lepidoptera). Acta Phytopathologica et Entomologica Hungarica, 44: 111-117.

- Palágyi-Mészáros, L., Balogh, J., Latinovics, D., Balogh, T., Klement, É., Medzihradszky, K., Rákhely, G., Kovács, K. L. (2009): Electron-transfer subunits of the NiFe hydrogenases in Thiocapsa roseopersicina BBS. FEBS Journal, 276: 164-174.

- Papdi, Cs., Joseph, M.P., Pérez-Salamó, I., Vidal, S., Szabados, L. (2009): Genetic technologies for the identification of plant genes controlling environmental stress responses. Functional Plant Biology, 36: 696-720.

- Tamás, C., Némethné Kisgyörgy, B., Rakszegi, M., Wilkinson, M. D., Yang, M. S., Láng, L., Tamás, L., Bedô, Z. (2009): Transgenic approach to improve wheat (Triticum aestivum L.) nutritional quality. Plant Cell Reports, 28: 1085-1094.

- Turóczy, Z., Kis, P., Török, K., Cserháti, M., Lendvai, Á., Dudits, D., Horváth, V. G. (2009): Improvement of the oxidative and heat stress tolerance in transgenic tobacco by the overexpression of an ABA induced aldo-keto reductase from rice. Planta, 211: 693-700.

2010

- Beczner, F., Dancs, G., Sós-Hegedús, A., Antal, F., Bánfalvi, Zs. (2010): Interaction between SNF1-related kinases and a cytosolic pyruvate kinase of potato. Journal of Plant Physiology, 167: 1046-1051.

- Bittsánszky, A., Gyulai, G., Kőmíves, T. (2010): Arabidopsis thaliana overexpressing Zea mays glutathione S-transferase: modelling an effective phytoremediation. Journal of Biotechnology, 150: Supplement 1, 486.

- Hiripi, L., Negre, D. Cosset, F-L., Kvell, K., Czömpöly, T., Baranyi, M., Gócza, E., Hoffmann, O., Bender, B., Bôsze, Zs. (2010): Transgenic rabbit production with simian immunodeficiency virus-derived lentiviral vector. Transgenic Research, 19: 799-808.

- Kvell, K., Czömpöly, T., Hiripi, L., Balogh, P., Kóbor, J., Bodrogi, L., Pongrácz, J. E., Ritchie, W. A., Bôsze, Zs. (2010): Characterisation of eGFP-transgenic $\mathrm{BALB} / \mathrm{c}$ mouse strain established by lentiviral transgenesis. Transgenic Research, 19: 105-112.

- Maróti, J., Farkas, A., Nagy, I. K., Maróti, G., Kondorosi, É., Rákhely, G., Kovács, K. L. (2010): A second soluble Hox-type NiFe enzyme completes the hydrogenase set in Thiocapsa roseopersicina BBS. Applied and Environmental Microbiology, 76: 5113-5123.

- Nagy-Gosztonyi, M., Nagy, A., Németh-Szerdahelyi, E., Pauk, J., Gelencsér, É. (2010): The activities of amylases and $\alpha$-amylase inhibitor in wide-range herbicide resitant wheat lines. Czech Journal of Food Science, 28: 217-224.

- Székács, A., Lauber, É., Juracsek, J., Darvas, B. (2010): Cry1Ab toxin production of MON810 transgenic maize. Environmental Toxicology and Chemistry, 29: 182-190. 
- Székács, A., Lauber, É., Takács, E., Darvas, B. (2010): Detection of Cry1Ab toxin in the leaves of MON 810 transgenic maize. Analytical and Bioanalytical Chemistry, 396: 2203-2211.

- Tamás, L. (2010) Molecular farming, using the cereal endosperm as bioreactor. Acta Agronomica Hungarica, 58: 55-64.

- Tóth, D. M., Szőke, É., Bölcskei, K., Kvell, K., Bender, B., Bôsze, Zs., Szolcsányi, J., Sándor, Z. (2010): Nociception, neurogenic inflammation and thermoregulation in TRPV1 knockdown transgenic mice. Cellular and Molecular Life Sciences (DOI 10.1007/s0018-010-0569-2).

$$
2011
$$

- Cervenak, J., Bender, B., Schneider, Z, Magna, M., Carstea, B. V., Liliom, K., Erdei, A., Bôsze, Zs., Kacskovics, I. (2011): FcRn overexpression boosts humoral immune response in transgenic mice. Journal of Immunology, 15; 186: 959-968.

- Darvas, B., Bánáti, H., Takács, E., Lauber, É., Szécsi, Á., Székács, A. (2011): Relationships of Helicoverpa armigera, Ostrinia nubilalis and Fusarium verticillioides on MON810 maize. Insects, 2: 1-11. (doi:10.3390/ insects2010001)

- Turóczy, Z, Kis, P, Török, K, Cserháti, M., Lendvai, Á, Dudits, D., Horváth, V. G. (2011): Overproduction of a rice aldo-keto reductase increases oxidative and heat stress tolerance by malondialdehyde and methylglyoxal detoxification. Plant Molecular Biology, 75: 399-412.

- Lichner, Z., Páll, E., Kerekes, A., Pállinger, E., Maraghechi, P., Bôsze, Zs., Gócza, E. (2011): The miR-290-295 cluster promotes pluripotency maintenance by regulating cell cycle phase distribution in mouse embryonic stem cells. Differentiation, 81: 11-24.

\section{List of authors and their contact details}

- Ervin Balázs, full member of HAS (Hungarian Academy of Sciences), Department of Applied Genomics, Agricultural Research Institute of the Hungarian Academy of Sciences (HAS), Martonvásár, balazs@mail.mgki.hu

- Zoltán Bedó, full member of HAS, Agricultural Research Institute of the Hungarian Academy of Sciences, Martonvásár, bedoz@mail.mgki.hu

- Zsuzsanna Bósze, Doctor of Science of HAS, Applied Animal Biotechnology Group, Agricultural Biotechnology Center, Gödöllô, bosze@abc.hu

- Dénes Dudits, full member of HAS, Institute of Plant Biology, Biological Research Centre, Hungarian Academy of Sciences, Szeged, dudits@brc.hu

- Miklós Gábor Fári, Doctor of Science of HAS, Department of Plant Biotechnology, Faculty of Agricultural and Food Sciences and Environmental Management, Centre for Agricultural and Applied Economic Sciences, University of Debrecen, Debrecen, fari@agr.unideb.hu

- Júlia Gimes, journalist, gimesjuli@freemail.hu

- Elen Gócza, Ph.D, Applied Animal Biotechnology Group, Agricultural Biotechnology Center, Gödöllô, elen@abc.hu

- János Gundel, Ph.D, Swine Nutrition Research Group, Research Institute for Animal Breeding and Nutrition, Herceghalom, gundel.janos@atk.hu

- László Hiripi, Ph.D, Applied Animal Biotechnology Group, Agricultural Biotechnology Center, Gödöllő, hiripi@abc.hu

- László Hornok, full member of HAS, HAS-SZIU-ABC Mycology Group, Faculty of Agricultural and Environmental Sciences, Szent István University, Gödölló, Hornok.Laszlo@mkk.szie.hu

- Kornél Kovács, Doctor of Science of HAS, Institute of Biophysics, Biological Research Centre, Hungarian Academy of Sciences, Szeged, kornel@brc.hu

- Csaba L. Marton, Doctor of Science of HAS, Maize Breeding Department, Agricultural Research Institute of the Hungarian Academy of Sciences, Martonvásár,martoncs@mail.mgki.hu

- László Márton, Ph.D, Károly Ereky Biotechnology Trust, Debrecen, drlaszlomarton@gmail.com

- Mária Oszvald, Ph.D, Department of Plant Physiology and Molecular Plant Biology, Eötvös Loránd University, Budapest, maria.oszvald@gmail.com

- József Popp, Doctor of Science of HAS, Research Institute of Agricultural Economics, Directorate of Agripolitics, Budapest, Popp.Jozsef@aki.gov.hu

- Katalin Posta, Ph.D, HAS-SZIU-ABC Mycology Group, Faculty of Agricultural and Environmental Sciences, Szent István University, Gödöllő, Posta Katalin@mkk.szie.hu 
- Norbert Potori, Ph.D, Research Institute of Agricultural Economics, Department of Agripolitical Research, Budapest, potori.norbert@aki.gov.hu

- Ingo Potrykus, honorary member of HAS, member of the Pontifical Academy of Sciences, Institute of Plant Sciences, Swiss Federal Institute of Technology (ETH), Zuerich, Switzerland, ingo@potrykus.ch

- László Sági, Ph.D, Plant Cell Biology Department, Agricultural Research Institute of the Hungarian Academy of Sciences, Martonvásár, sagil@mail. mgki.hu

- Béla Somfai, S. J. bioethicist, professor emeritus, Sapientia College of Theology of Religious Orders, Budapest, somfaib32@gmail.com

- László Tamás, Ph.D, Department of Plant Physiology and Molecular Plant Biology, Eötvös Loránd University, Budapest, tamasl@ludens.elte.hu

- Pál Venetianer, full member of HAS, Institute of Biochemistry, Biological Research Centre, Hungarian Academy of Sciences, Szeged, venetpal@brc.hu 